A-H [annover] Systematischen 1897 


\section{HARVARD UNIVERSITY.}

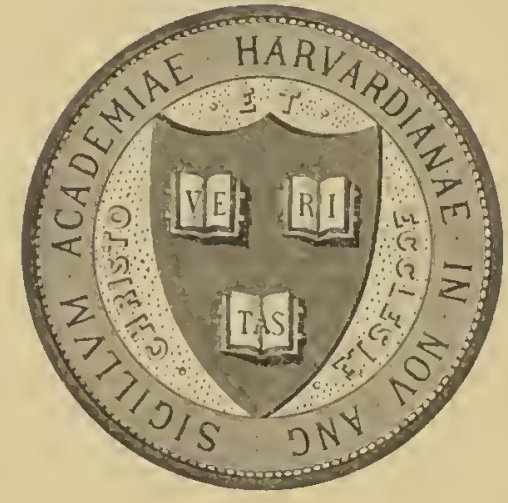

LIBRARY

OF THE

MUSEUM OF COMPARATIVE ZOÖLOGY.

$$
\text { SNengange }
$$

Shaguet $17,1898$. 
证

\section{มำ? $7189 \%$ \\ 14,199 Katalo8}

der

\section{systematischen Vogelsammlung}

des

Provinzial-Museums

in

Hannover.

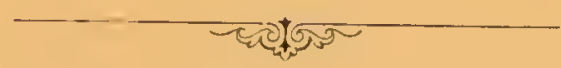

Hannover.

Druck von Wilh. Riemschneider.

1897. 



\section{Katalog}

der

\section{systematischen Vogelsammlung}

des

Provinzial-Museums

in

Hannover.

Hannover.

Druck von Wilh. Ricmschneirler.

1897. 



\section{Vorwort.}

Die Vogelsammlung des himmoverschen Provinzial-Jluseums zerfitlt in drei Teile, deren rrster die prosse Schau-Sammlung les in Göttingen rerstorbenen llofrat Neyer umfasst, seiner Zeit ron sr. Mlajestiit dem Könige Georg angekauft und den Simmlungen der naturhistorischen Ciesellschaft leihweise überlassen, später ron Sr. Könighichen Hoheit dem Herzoge von C'umberland dom hamnoverschen Provinzial-Museum in gleicher Wrise belassen. Dirse Sammlung ist gruppenweise in eleganten schaukästen aufgestellt und hat ihre schrankweise aufgestellten Verzeichnisse. Die einzelnen Yögel sind mit Nummern bezeichnet. Der vorliegende Katalog enthillt die Namen der Vöggel der systematischen Sammlung, die Anzahl der Exemplare jeder Art, dir Herkunft und die Namen der Sr.henkgeber. Die zweite eingeklammerte Nummer. die auch jedes Exemplar trägt, ist die Nummer des Katalogs der Vogelsammlung des Senckenbergschen Musenms in Frankfurt a. M., dem auch in der Einteilung und Aufstellung gofolgt ist. Diese Nummern sind, übereinstimmend mit den Nummern in der Meyersehen Sammlumg, auf farbigem Papier angehracht. um sichtlich den Erdteil der Herkunft zu hezeichnen. lis berdeutet weiss kuropa, grün Amerika, blan Asion, gelb Afrika und rot Polynesien. Die dritte Abteilung der Vogalsanmlung, die Vögel der l'rovinz Hannover erhailt ihren eigenen Kiltalog.

Hannorer im Juni 1897.

Dr. Riist. 



\title{
Ordo Passeres.
}

\author{
Subordo Oscines.
}

\section{Familie Turdidae. \\ Unterfamilie Turdinae.}

\section{A. Lusciniformes.}

1 (2). Ä̈don luscinia (L.) Mandtigndl.
a. O' Hamnover. (i. v. C. Braunstrin.

2 (4). Cyanecula cyanceula (Wolf.) Beijiteruiges BInu fefildjell.

a. O' Hannover. Ciöttingen, Museum.

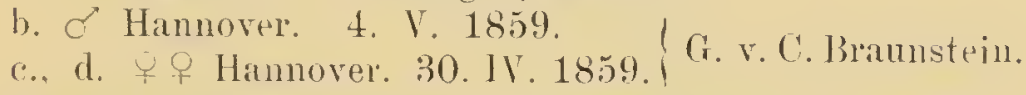

3 (7). Erithacus rubeculus (L.) Rotfefldaeu.

a. c' Hannover.

b. pull. Hannover. (i. v. C. Braunstein.

c. F Hannover.

4 (8). Ruticilla tithys (L.) Şaแลrotjdunแj.

a. 7 Hannover. 21. IV. 1858. G. v. ('. Braunsein.

b. jur. Hannover. (i. v. linst Hahn.

Ђ) (9). R. phoenicurus (L.) Barteurotjdiwaus.

a. C) Hannover. 2. V. 1858.

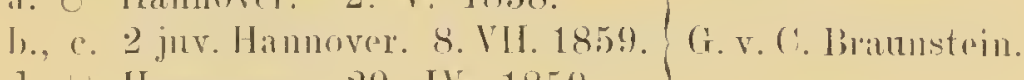

d. F Hannover. 29. IV. 1859.

(i (16). Accentor modularis (l.) Secteubramelle.

a. of Hannorer. (i. v. (Є. Braunstrin.

b. jur. Ina\% (i. v. I). Vïhlemptorll.

7 (17). A. collaris (ticop.) Mrpenbramelle. a., b. ó o' schwri\%.

co lialer.

s (18). Pratincola rubetra (L.) Riejcujdutülact.
a. d Hannover.

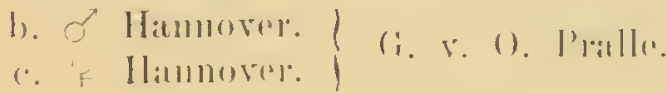


9) (19). Pratincola rubicola (L.) Edjunrofehliger Biejeu= (đ)

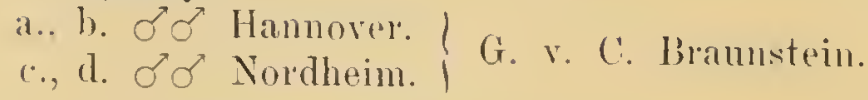
1 Balg.

10 (29). Petroeca phoenicea, Gould.

a., b. $\sigma 0$ Anstralien, Tasmania. ! (i. v. Konsul

$\because$ O Australien, Tasmannia. i Kanffmam.

d. of Australien. G. v. Exce. v. Malorti.

11 (29a). P. erythrogastra, Gould.

i. O Australien. G. v. Konsul Kauffmamm.

12 $(29 h)$. P. rhodinigastra, could.

a. of Anstralient.

$13(29$ r). P. bicolor, Swains.

a. O Anstralien.

14 (29 d). P. multiculor, Swains.

a. ơ Australien.

15 (29e). P. Goudenowii, Jard. r. Selb.

i., b. ठव Anstralien.

16 (30 i). P. fusca, Goull.

a. of Australien.

17 (31). Miro albifrous (6im.).

a. S Australien. (i. v. Konsul Kantfmann.

18 (32). Saxicola oenanthe (. .) Stcinjdunäzcr.
a. of Hannovir.
b. of Goslar. G. v. C. Braunstein.
a. ㅇ Goslar.
d. of jur. Hammover. (i. r. C. Rüst.
r. O Hamover. (i. r. Mr. Hahne.
o lial w.

19 (35). S. stapazina (1..) Dhrenjtcinjofuņ̨̈er.
a. Stmyma.

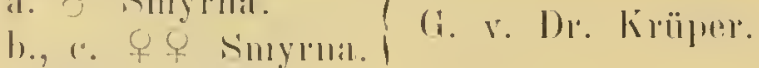
d. of Portugit.
r. Cl Furopa. (i. v. Gimaf v. d. Sichulenburge.
2 liil|ge.

20 (37). S. lugens (Lichlit).

i. Siiil-Rinssiaturl.

21 (40). S. isabellina ((1ret\%s+hmo).

i. jur. Sulyluia.

2.) (+1). S. lencura (Gm.).

i. of jux. Aregytur. 
h. Areypten.

liilly.

$2: 3(+2 a)$. S. aurita ('trmm.).

a. Gl Europil. (i. r. (ir. v. d. sichutembure.

h. f smyrna. (i. v. H)r. liriipre.

$2+(42 \mathrm{~b})$. Origma rubicata (Gould).

a. Anstralirin.

25 (51). Sialia sialis (L.).

a., b. उo Nordamerikis.

(.) O Nordamerikit.

of bialy.

26 (52). Mouticola saxatilis (L.) Etcindrofiel.

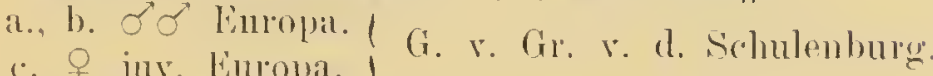

d. Ot Europa. G. v. C. Braunstein.

27 (5:3). M. cyanus (L.) Blaubruifel.

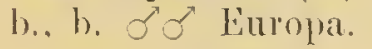

(c. jur. Liuropa.

d. of Europia.

$28(5 \overline{7}) . \quad$ II. erythrogastra (Vig.).

a. Indien.

29 (58). M. solitaria (Müll.).

a. Westimdien.

\section{B. Turdiformes.}

30 (60) a). Geocichla interpres (Bonю.).

a. J Javiı.

b. Java.

c. I Java.

31 (62). (6. Iunulata (Lath.).

a., b. Australien. (i. v. Konsul Kinffmann.

32 (71). Merula merula (1..) amiel.

a. J Hannover.

b. 8 Hannover.

(.) Hammover.

g. jus. Hammoser. (i. v. H)r. Mathu.

h. Hammovire.

3: (73). M. tolquata (I.) Miugoroifer.

a. J Kicuenturt. (i. v.

b. $\sigma$ Hammorer. (i. v. M)r. Halun.

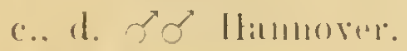

a) Catlenbury. G. v. Obriantm. Liteder. 
34 (б). M. atrigularis (Trmm.) Bedjiteius Droijer.

i. T Göttingen.

35 ( 79 a). M. fuscater, Lafr.

a. ㅇ Mexiko. G. v. Obrrhofü̈rtne Wendland.

36 (79 b). M. chiguana, Lafr.

a. Nexiko. G. v. Oberhofurither Wendland.

37 (80). Turdus pilaris (L.) Madljorderoroillel.

a. $\sigma$ Mellendorf. G. r. G. Kreye.

b. \& Hannover. G. v. Dr. Voigt.

(r. \& Hannover.

d. Nest. G. v. H. Kireye.

ㅇ Balw.

38 (81). T. viscivorus, L. Mitferoroijel.

a., h. of Hamnover.

(. G Goslar. G. v. C. Bramstein.

d. Cl alh. Hammorer. (i. v. G. Glimmann.

e. alh. Göhırle. G. v. Oherförster Wallmann.

39 (8:3). Turdus iliacus, 1. Rotbroijel.

a., b. ơ Hamover.

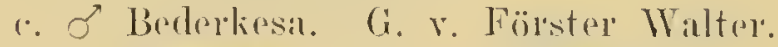

d. F Hammover. G. v. G. Bohne.

e. \& Hannover.

40 (84). T. musicus, l. Siugdroifer.

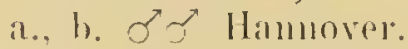

(. O Hamnover. 13) 1]. 1865. (i. צ. C. Braunstein.

d. Of Hammover 1862. (i. v. (\% Braunstein.

a. Of Hannover. (i. v. Burkharelt.

f. of Göhrde. (i. v. Wildne. Gireselmamn.

g. Bordenan. G. . Wildm. Wallnamm.

h. \& Hammover.

41 (85). T. mustelimus, Gim.

a. of Norrimerikit.

b. Nolrlamorikil.

42 (8s). 'T. obseurus, Gimel.

a. K Kilmschatkal. 2.jlll. 1887.

$4: 3$ (90 a). T. smithi, himp.

a. Afrikil mer. (i. v. Mr. Mleyer.

If (92). T. magellaniens, King.

i. of ('hili laldivia 18603.

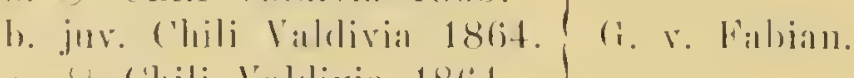

(.) C Chili Vialdivia 1864.

(i) (94: a). T. nammanmi, 'T'(m).

a. l'suri. 16./X́l. 1889. 
46 (45). T. migratorius, L. Manderorofiel.

a., b. $\gamma^{2}$ Nordamerika.

r. P Nordamerikil.

47 (96). T. fumigatus (Licht).

a. Brasilicn.

48 (97a). T. ferrugineus, I'. Wierl.

a. Mrikio.

49 (98). T. gymmophthalnus, Cah.

a., h. Surinaul.

50 (99 a). T. ruficollis, Pall.

a. of Kultuk, Baikal.

51 (100). Mimus polyglottus (L.) Spottorofifcl.

a., b. б์ ơ Nordamerika.

52 (101) M. hypoleucus, Bonp.

a., b. ơ O' Nlexiko.

53 (106) Cinclosoma punctatum (Lath).

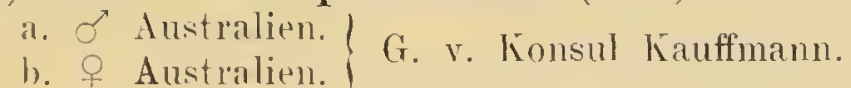

ơ Balg.

$54(107)$. Donacobius atricapillus (L.).

a. Brasilien.

b. Brasilien. G. v. Dr. Toelsner.

55 (108). Harporhynchus rufus (L.).

a. Nordamerika.

Unterfamilie Copsychinae.

j6 (114). Copsychus mindauensis (Gm.)

a., h., r. $\sigma \sigma \sigma$ Mlalakka.

d. of Borneo.

e. jur. Java.

57 (140). Myiophoneus cyameus (Horsf.) a. Javí.

j8 (141). Cettia cetti (Narm.)

a. $\sigma^{2}$ sinyma.

1. f Neamanien.

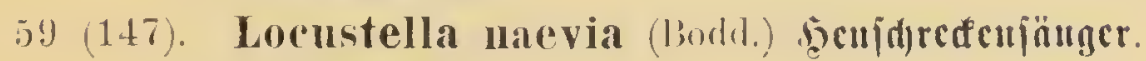

a., h. Ilannover.

(2) Hannover. 1869. (A. v. Pralle.

(j0) (14r). L. luscinioides (Savi) Madjtignflrolgrjünger.

a. O' Europa.

b. $\sigma$ Italien. 
61 (150) Calamodus aquaticus (Temm.).

a. O' Deutschland.

62 (151). C. schoenobaenus (L.) Edjilfrohrjäuger.

a. of Hannover. G. v. C. Braunstein.

b. o Hannorer. G. v. I'ralle.

63 (15\%). Acrocephalus arundinaceus (L.) Droficlrohr= jüug̣cr.

a. O Hammover.

b. \& Hannover.

64 (156). A. palustris (Buclst.) Sumpfiäuger.

a. of Hannorer. (i. v. P'ralle.

65 (157). A. streperus (Vieill.) Tcid)roljrjänger.

a. of Hammover.
b. \& Hannover. G. v. ( $\quad$ Braunstein.

66 (160). Hypolais philomela (L.) Giartenjpötter. a., b. ठठ ठ Hannover.

67 (162) H. pallida (Ilempr. wh lihr).

a. Oo spanien.

b., c. Smyrma.

b8 (163). Phylloscopus sibilator (lieclist.) 2ialoland= jänger.

a. Of Hamnover. G. v. Dir. Niemeyer.

h.. r. of Hamover. G. r. ('. Braunstrin.

(69) (166). Ph. trochilus (I.) Fitiămubiün!̣er.

a. of Hannover. G. v. C. Braunstrin.

h. Of Hamover. G. I. I'ralle.

c. H Hannover.

70 (168). Ph. rufus (liechst.) 2Bcioculaubugat.

a., b. of Hammoror. G. v. (! Braunstein. o lialy.

71 (170). Sylvia nisoria (Ber.lıst.) Sperbergragిmilfe.

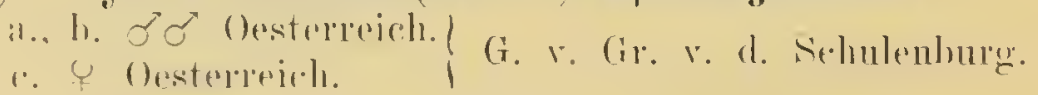

d. H Hannover. 1862. (i. v. C. IBraunstein.

72 (171). S. orphaea ('Temm.) Ë̈ngergrasึüife.

i. of sïtfrankreich.

73 (172). S. melanocephala (Gm.).

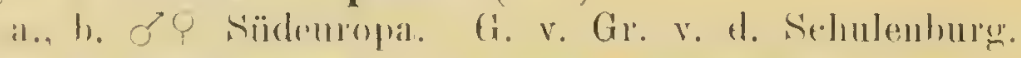

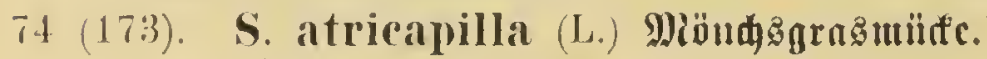

a. Of Haunover. 1865. G. v. G. Braunstein. 
\begin{tabular}{l|l} 
b. Jur. Hanmover. \\
․ Hom Hover. 1865.
\end{tabular}

$75(174)$. S. rïppelli ('T'mm.).

a. Simyina.

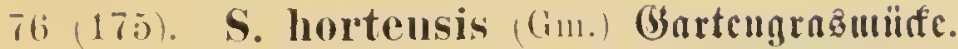

a. O Hammorer. 20. VIl. 1858 . (i. v. P'alle.

b. all, Hannover. G. v. (Y. Branustein.

77 (176). S. curruca (I.) Baแugrazunitfe.

i. of Hammover.

78 (178). S. Sylvia (L.) Durugrazmuinte.

a. Of Hannover. G. v. (). Bramustein.

79 (180). S. conspicillata, Marm. Briffenjüuger.

a., b. бo $\sigma^{7}$ Portugal.

(.) Portugal.

80 (181). S. subalpina, lionelli.

a. O' Italien.

b., c. $0^{7} \sigma^{7}$ Griechenland.

81 (182). S. undata (Bodd.).

a. o Südeuropa. ( G. v. Gir, v. d. Schulenburg.

$82(185)$. S. galactodes, Temm.

a. $\sigma$ Kleinasien. 12.N. 1877.

b., $\bullet$ o of Kleinasien. G. v. Gr. v. d. Schulenburg. ơ diälge.

of baly.

\section{Familie Timeliidae.}

\section{Unterfamilie Troglodytinae.}

83 (192 a). Thryothorus palustris, Bonp.

a. Norrlamerika.

84 (192h). Th. platensis, ('ah.

a. Nurinan.

$85(192 \cdot \cdot)$. Th. coraya, vieillot.

a. Brasilien. G. v. Br. T'̈̈lsmer.

$86(195$ a). Troglodytes hiemalis, Viellot.

a. J Norrlamerika.

87 (1999). Anorthura troglodytes (1.) Zaunfüig. a., b. бơ Hannover.

1 Baly. 
88 (205). Cinclus aquaticus (Bechst.) $\mathfrak{B n}_{\text {niferitut. }}$

a. G Galizien.

b. Of Hannover.
c. jur. Hannover. (i. v. C. Braunsterin.

d. ㅇ Hannover. G. v. Krïper.

89 (206). C. asiaticus, tw.

a. đo jur. Jira.

\section{Unterfamilie Cisticolinae.}

90 (208). Malurus malanocephalus, Vig. et Horsf.

a. Australien. G. v. I'rof., Dr'. Mü̈ller'.

91 (208a). M. elegans, (iould.

a., b. Australion. G. v. Konsul Kauffmann.

92 (209). M. browni, Vim. ut Horsf.

a. Anstralien.

9:3 (226). Cisticola cisticola (Trmm.).

at. O'smyrna.

b. of smyrnit.

9) (2:36 a). A canthiza chrysorrhoca, (sray.

a. Anstralien. G. v. l'rof., Nü̈ller.

h. Iustralien. G. v. Kons. Kanffmamn.

\section{Unterfamilie Timelimae.}

95 (256). Psophodes (exepitans (Lath.).

a., h. Lustralimn. (i. v. Kons. Kauffmann.

96 (287). Pomatorhinus montanus, Horsf.

a. (eylon.

97 (287a). P. rubeculus, Gould.

a. Australien.

98 (2s9 a ). P. supepoiliosus, Vig. et Horsf.

a. Australien.

99 (2s:) h). P. ruficeps, Hartlanh.

a. Australien.

100 (313). Mixoluis melanothorax (Ttmm.).

a. Javiı.

101 (315). Macronus ptilosus, Jard.

a. Ostindien. 


\section{Familie Paridae \\ Unterfamilie Regulinae.}

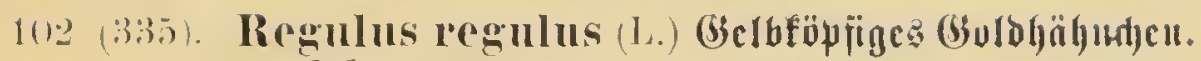

a., h. of o Hannover.

(.) Y) Hannover.

103 (336a). Cyanotis azarae, Gray.

a. (hili. G. v. Fabian.

10) (337). Regulus ignicapillus (Temm.) Fencrüpïiges (Guldhähud)en.

a. I Hammover. 1865 . G. v. Brannstein.

Unterfamilie Parinae. 1Hıiliu.

105 (3 41 ). Panurus biarmicus (L.).

a. O Russland.

b. 우 Russland.

106 (34:3). Aegithalus pendulinus (L.) Bentclucije.

a. 'o Hamover.

b., c. of of Hannover.

$10 \bar{T}(4+5)$. Acredula caudata (L.) Edjunuzmcife.

a. ơ Hamnover.

b. † Hannover.

108 (347). Parus cristatus (L.) Şmubeumcije.

a. C Hannover. (i. v. Obergerr. Dommes.

b. \& Hannover. G. v. Dr. Nejer.

109 (351). P. palustris, L. Emmpfucije.

a. jur. Hannover.

b. \& Hannover.

110 (35) 4 . P. lugubris, Natteper. Traucrucife.

a. of Dalnatien.

111 (358). P. major, I. Sohglmcife.

a. of Hannover. (i. v. Obergerr. I)ommes.

b. O' Hannover. G. v. Mr. Hahn.

r. of Hannover. G. v. ('. Braunstrin.

112 (360). P. ater, L. Tnumcumcife.

a. of Hannover.

11:3615). P. coeruleus, L. BIanucife.

a. J IIannover.

b. T Hannover.

or Balg. 
11+ (362). P. cyanus, Pall. Eajurnteije. a. O Russland.

115 (363a). P. amabilis, sharpu.

a. Of Ostasien.

$116(362 h)$. P. cinerens, Virill.

a. of borneo.

\section{Familie Certhiidae. \\ Unterfamilie Sittinae.}

117 (370). Sitta caesia, Wolf. Silcilucr.

a. of Hannover. (G. v. Dr. Mlejer.

b., $\because$ o o Hannover.

$118(372)$. S. newmayeri, Nichahell.

a. o' Smyrna.

$119(37 ; 3 a)$. S. kniperi, v. Pralu.

a. F Kleinasien.

12()$(375)$. S. frontalis, Swains.

a. Java. G. v. (? Braumstein.

$121(376)$. S. azurea, lass.

a. Java. (i. v. Götting. Nuscmm.

122 (378). S. chrysoptera (Lath.).

a. Australien.

h. Australien. (i. v. P'rof. I)r. Müller.

2 bälgge.

\section{Unterfamilie Certhinae.}

12:3 (380). ('limacteris lencophaca (Lath.).

a. Australien.

12.4 (382). Tichodroma mularia (l.) Minucrlünfer.

a. 8 sicliweiz.

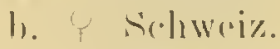

4 Biil

125) (38:3). Certhia familiaris, 1. Bammläufer.

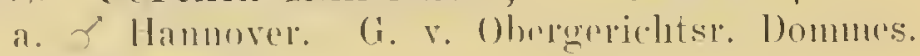

b. Hannover.

\section{Familie Dacnididae.}

Unterfanilie Jicaeince.

126 (385). Pardalotus pumctatus, shaw.

a., h. Nustralien. (i. v. Konsul kauffmann.

7 Bälge. 
127 (385)a). P. aftinis, ciould.

a. Austrilien. (i. v. Konsul kinffurann.

128 (385b). P. striatus, Vim. ot Horsf.

it, h. 2 juv. Austialime.

1.9) (391). Dicaenu hirundinacemun (Lath.).

a. O Australion.

\section{Unterfamilie Dacnidinae.}

130) (411). Dacnis cyanomelas, ('ih.

a. of Brasilien.

1). Brasilien. K. k. (Könighliches Eigentum jetzt liigentum des Herzogs ron Cumberland).

2 biilge.

$131(+12)$. Chlorophanes spiza (L.).

a., b. ơㅇ brasilien.

13. (414). Coereba cyanea (L.).

a., b. $\sigma^{\circ} \sigma^{\circ}$ Brasilien.

(๖, o jur. Brasilien.

d. ㅇ Brasilien. (i. v. Dr. Tölsner.

133 (416). Certhiola chloropyga, Cah.

a. Brasilien. G. v. Dr. Dommes.

134 (417a). G. flaveola, Licht.

a. Brasilien. G. v. Br. Toelsner.

\section{Familie Nectariniidae.}

135 (419). Nectarinia famosa (Lin.).

a., b. Afrika. (i. v. Dr. Mejer.

(.) Afrika. G. v. Frln. v. Poten.

136 ( 419 a). N. australis, Ciould.

a. \& Australien. G. v. Konsul Kauffmann.

137 (4:31). Cinnyris chalybaea (L.).

a. Afrika. G. v. G. Löwentlat.

138 (4:31a). C. platyura, Vieill.

a. Afrikit. (i. v. G. Löwentlual.

b. of Afrika.

139 (4333). C. pulchella, (ah).

n., b. Afrikir.

(A) Afrika. G. v. G. Jöwenthal.

$140(4: 333$ b). C. splendida, Sllaw.

i. Afrika. G. v. G, löwenthal. 


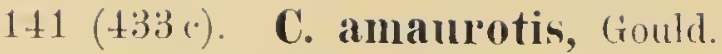

a. $\sigma$ juv. Java.

142 (443). C. senegalensis ( $\left.\mathrm{L}_{\text {. }}\right)$.

a. O Atrika. G. v. G. Löwenthal.

143 (447). Arachnothera Iongirostris (Lath.).

a. $\sigma$ sumatra.

$1+4$ (449). A. affinis (Horsf.).

a. Java. (i. v. (.) Briunstein.

$145(454)$. Promerops cafer (L.).

a. Siidafrika. Götting. Museum.

\section{Familie Meliphagidae.}

146 (456). Myzomela sanguinolenta (Lath.).

i. of Australien.

147 (458). Acanthorhynchus tenuirostris (Lath.).

a., b. Australien.

148 (468:1). Melithreptus virescens, Vieill.

a. Australien. (i. v. Mr. Miiller.

14!) (471). Meliphaga phrygia (Lath.).

a. Anstralien. G. v. Kousul Kauffmann.

150) (471:1). M. australasiana, shaw.

a. Australien. (i. v. Kousul Kauffmann.

151 (472). Ptilotis auricomis (Lath.).

a. Australien. G. v. Kousul Kauffmann.

b. Anstralien. (i. v. (iröner.

152 (475). Pt. somora (Gould).

i1. Anstraliens.

153 (477). Pt. flavigula, (ionld.

a. Anstralien. (i. v. Konsul Kanffmann.

154 (478). Pt. leucotis (1ath.).

a. Australien. (i. v. Konsul Kanffmamm.

155 (483). Meliornis novaehollandiae (tath.).

a. Australien. G. v. Konsul Kauffnann.

1.56 ( 187 ). Manorhina melanophrys (Lath.).

a. Austrialionl.

157 (4si). M. garlula (latho).

i. of Austrilion.

b. Australien. (i. v. Konsul Kanffinamm.

c. Australien. G. v. Exce v. Malorti. 
158 (4S8 11$)$ Myzantha. (?)

a. Jitial.

159) (459). A('anthochaera inauris (Gould).

a. Australien. G. v. Exe. v. Natonti.

b. \& Tasmanien.

160 (490). A. calunculata (Latle.).

a. Australien. (i. v. lixe, v. Malorti.

161 (491). A. mellivora (Lath.).

a. Australien. G. v. Konsul Kauffinann.

b. Australien.

162 (493). A. rufigularis (Gould).

a. $\sigma$ Australien.

b. ㅇ Australien.

163 (494). Entomyza cyanotis (Lath.).

a., b. $\sigma^{7} \mathcal{O}^{7}$ Australien. G. v. Dr. Mülller.

164 (495). Philemon cop'niculatus (Lath.).

i. Australien. (i. v. Konsul Kauffmann.

\section{Familie Brachypodidae.}

165 (496). Chloropsis nigricollis (Vieill.).

a. Malakka.

166 (5)1). Ch. icterocephala (Temm.).

a. Malakka.

167 (511). Hemixus virescens (Temm.).

i. F Java.

168 (513a). Pycnonotus (Microtarsus) lencogrammicus, Miiill.

a. Sumatra.

169) (514:1). P. chalcocephalus (Temm.).

a. Java.

170 (515). P. aurigaster (Vieill.).

a. Jaท⿰.

171 (517). P. xanthopygus (Hempr. of Ehr.).

a. Afrikil. (i. v. Dr. Rocholl.

172 (522). P. bimalculatus (Horsf.).

a. Javil.

173 (538). Trachycomus ochrocephalus (fim.).

a. Javia.

174 (541). Microtarsus melanocephalus (iur.).

it. Jilvil. 


\section{Familie Alaudidae. Texthent.}

175 (542). Otocorys alpestris (L.) If(penlerdje.

a. of Sichweiz.

176 (545a). Geositta (Certhilauda) cunicularia, Kittlitz.

a. Costarica.

177 (548). Nelanocoryphal calandra (L.) finlamberferdje.

a. of Europa.

1)., ('. 우 Finropa.

178 (5.5:3). Alauda arvensis (L..) Felolerdje.

a. Of Himnover. (G. v. I)r. Voigt.

b. of Hannover. (i. v. O. Pralle.

a. \& alb. Springe. G. r. Forstm. Hexse.

d. Hamnover. G. r. Gr. v. d. Schulenburog.

e. Y Hannover.

179) (5)66). Calaudrella brachydactyla (Leisl.).

a. süideuropa.

$180(560)$. Mirafra javanica, Horsf.

a. o Java.
b. J Jara.
c. Javal.

181 (561). M. horsfieldi (Ramsay).

a. Australien. G. v. G. Gröner.

182 (5)(63). Galerita cristata (1 .) Jenubulerdje.

it, b. ₹f Hammover. (G. v. (). Pralle.

183 (563a). G. nigricans, C. L. Brehm.

i. $\sigma^{2}$ Aegyptrin.

$18+$ (j)66). Lullula arborea (1..) gecidelerdje

i. Oे Hannovel'.

b. Hamnover.

18.) (5-1 a). Pyrohulauda australis, smith.

a. Dustralien. G. v. G. (iroinese.

\section{Familie Motacillidae.}

186 (574). Maceonyx flavicollis (liiip).

a. Afrika. (i. v. (i. Isïwrintlal.

187 (5). M. capensis $\left(\mathrm{l}_{0}\right)$.

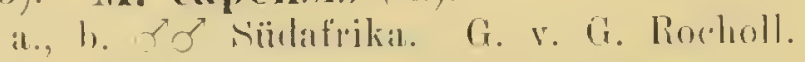

188 (577). Anthus trivialis (L.) Bammpieper

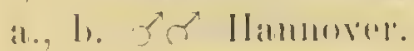

(․ jux. Hatlutorel. 


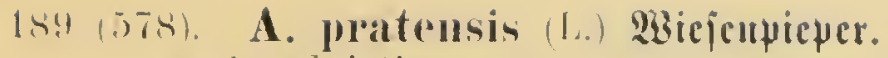

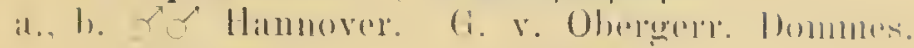

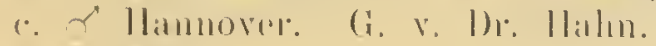

(I. of Goskitr.

r., f. tq Guslar.

$190(5,3)$. A. campestris (L.) Bruduper.

a. O Himnover.

191 (583a). A. rufeserns, Vig. of Morsf.

a. Australien.

192 (586). A. Spipoletta (L.) MBnjiferpicper.

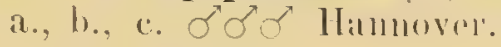

d. juv. Goslas.

c. P. Hannover.

1 Balg.

193 (5s6a). A. fuligimosus, Gould.

a., b. Australien.

$19+$ (588). A. australis, Vir. et Horsf.

a. Australien.

195 (591). Budytes flavus (L.) Bielbe Bandjef

a. Juv. Goslar. G. v. C. Braunstein.

196 (598), Motacilla boarula (L.) Geluirgagudjutelze. a., b. Hannover.

$197(600)$. M. alba, L. $\mathfrak{B c i p z e ~ B a d j i t e l z e . ~}$

a. o alb. Hamover. G. v. Dr. Armbrust.

\begin{tabular}{ll|ll} 
b. Of Hannover. ( G. v. C. Braunstein. &
\end{tabular}

198 (614). Grallina australis, Gray.

a. $\sigma$ Australien.

\section{Familie Mniotiltidae.}

199 (618 $a)$. Mniotilta aestiva, (iray.
a. surinam. K. li.
h. Mexiku.
(.) of jux. Drexiko.
d. f Surinam. K. F.

200 (6.21). Compsothlypis americana (L.)

a. 'f Norclimmerika.

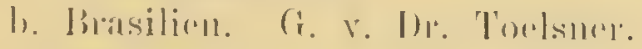

201 (6202). Dendrocca castaneal (Wik.).

a. Nordintrrikit. 
202 (625). D. discolor (Vieill.).

a. $\sigma$ Nordamerika.

b. $\sigma$ juv. Nordamerika.

c. $q$ Nordamerika.

203 (630). D. caerulescens (Gm.).

a. Noldamerika.

$204(632)$. D. coromata (L.).

a. Nordameriki.

205 (632 a). D. petechia, Gray.

a. Mexiko.

$206(633)$. D. aestiva (Gm.).

a. of Nordamerika.

b. Nordamerika.

207 (6336). Geothlypis trichas (L.).

a. Nordamerika.

208 (644). Setophaga ruticilla (L.).

i. Nordamerikir.

209 (649). Myiodioctes mitratus (Gm.).

i. of Nordamerikit.

\section{Familie Tanagridae.}

Unterfamilie Procniatinae.

210 (652). Procuias tersa (L.).

a. $\sigma$ Brasilien.

1. of Brasilien.

2 Biilger $\left(\begin{array}{ll}1 & \sigma\end{array}\right)$

Unterfamilie Euphoninae.

211 (653). Euphonia flavifrons (stratrm.).

a., 1. 50 Brasilien.

;) Biilge.

212 (65). E. nigricollis ((iin.).

a. of Brasilien. (i. r. Mr. Tölsmer.

213 (65)6). E. rufiventris (Virill.).

a. \& linasilinu.

2 biilge.

$21+(661)$. E. violacea (h.).

a. liogoti.

215 (bitio a). E. musi(al, Desm.

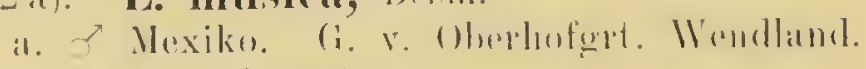
5,2 일. 
2ul6 (6is:). Chlopophonia viridie (Vivill.).

a. Birasilicon.

\section{Unterfomilie Tanu!rrinae.}

217 (bibi). Calliste tatan (L.)

a. Brasilien. (i. ve Ilofrat Burdhuld.

218 (6(i9). C. tricolor (im.).

a. Iiraxilien.

219 (672). C. thoracica (T'mun.).

a. Brasilien.

220 (675a). C. gypola, Gray.

a., b., c.. d. liasilin (a., b. K. E.).

3 Biilur.

221 (678). C. brasiliensis (L.).

a. Bratsilien. (๖, v. Dr. Tülsner.

b.. c., d., e. Brasilien (d., e. K. L.).

222 (679 a). C. Vassori (Gray).

a. Bogota. G. r. Cohn.

$223(679$ b). C. melanonota, Selat.

a. O' Brasilien.

224 (681). C. flava ( $(\mathrm{mm}$.$) .$

a. Brasilien.

2 Biilge.

225 (682). ('. xanthocephala (Tsch.).

a. $\checkmark$ Ecuarlor:

$226,685 \%$ Tanagrella velia (L.).

a. S lirasilien. 1857. G. v. D) Tölsmor.

227 (689). Buthraupis eucullata (Jart.).

a. Liasilion. (i. v. I)r. Tölsuer.

228 (69)1). Tanagra episcopus (L.).

a. Brasilien.

b. Y limsilien.

5 Biilge.

229 (691 a). T. archiepiscopus, Desm.

a. Mexiko. (i, v. Obremert. I)ommes.

230 (693). T. subcincrea, selat.

a. Mexilio. (i. v. Oberionfigt. Woudland.

231 (69) 4 a). T. capistrata, spix.

a. Brasilien. (i. v. M)r. T'älsmer.

232 (695). T. palmarum, l'r. v. Winl.

ง.. 1.. เ. Biracilien. 
233 (699). T. darwini, Bonp..

a. Eeuador.

$234(699$ a). T. nigerpina, Gim.

a. Brasilien. G. v. Dr. Tülsner.

235 (700). Rlauphocoelus brasilius (L.).

a. o Brasilien.

b. of Brasilien.

d. juv. Brasilien.

3 Bälge (darmenter 1 o und 1 o Balgr).

236 (704). Rh. jacapa (L.).

a., b. Brasilien.

237 (705). Rl. passerini, Bonp.

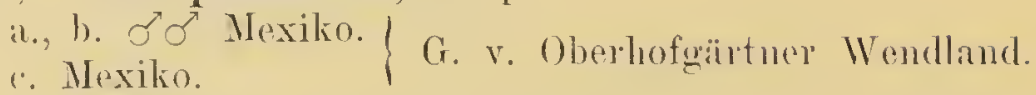

238 (706. Spindalis petrei (Less.).

a. Cuba. G. r. Colnt.

239 (712). Pyranga aestiva, Gm.

a. Virginien. G. v. Pinstor Mertens. 1 Balg.

240 (715). P. lubra (L.).

a. Nordamerika. G. v. Pastor Mertens.

241 (716). Ortlogonys vilidis (Spix.).

a. Brasilien.

242 (717). Phoenicothraupis rubica (Vicill.).

a. Brasilien.

243 (723). Tachyphouns cristatus (Gm.).

a., h. Brasilien.

1 Balg.

244 (725) T. coronatus (Vicill.).

a., b. Biasilien.

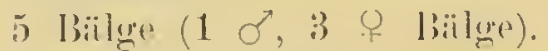

245 (725a). T. Ielleophateus (Vieill.).

a., b. Surinam. K. li.

246 (727). Poecilotluaupis lumulata (Du Bus.).

a. Sïdamerika.

247 (7:31). Pyrrhocomal louficeps (Strickl.).

a. Brasilien.

248 (73:). Nemosia pileata (Bocld.).

a., a. ơ Brasilimn.
r. of Brasilien 


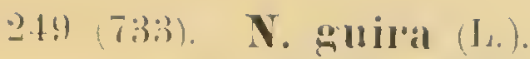

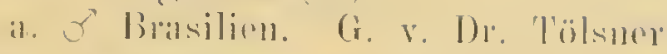

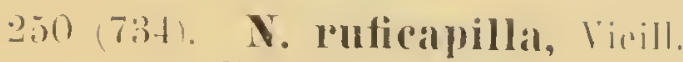

a. Brivilinen. G. r. Mr. Tölsmer.

b. Brisilian.

$251(73 \vdots))$ N. flavicollis, Virill.

a., b. उत Brasilien.

25) (736a). N. melanoxantlia (L.).

a. Brasilien.

\section{Unterfamilie Pitylinae (Arremoninae).}

253 (743). Pipilo erythrophthalmus :L.).

a. ơ Nordamorika.

b. of Norflamerikir. G. v. Dr. Nimeyor.

$254(745)$. Arremon silems (Bodd.).

a. ơ Brasilien.

b. Amerika.

c. Guiana. G. v. I)r. Tölsner.

1 Balg.

$255 \quad(760)$. Pitylus canadensis (L.).

a. o Brisilient.

b., c. Südamerika.

\section{Familie Fringillidae.}

Unterfamilie Emberisinae. Ammmlin.

256 (763). Emberizal schoenichus, L. Sivgrammer.

a. ơ Hamnover.

b. OJ Hannover. G. v. C. Braunstein.

c. of Hanmover. (r. v. Kanfunam Behne.

d. Hannover (Dollherem). (i. v. (), Riist.

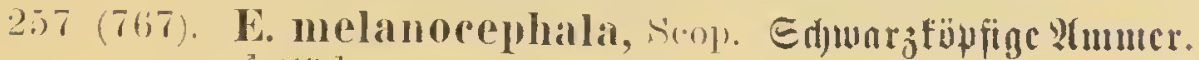

a. ơ Niideuropa.

b. 7 Sinyma.

258 (769 a) E. cinclea, Strickl.

a. of Simyrua.

h. S. simyrua.

$259(770)$. E. citrinella, L. Golonnencr.

a. O Hammovel.

J. Of Gostar. 1850. G. v. C. Dimanstrin.

c. Ilammorer. (i. v. l'ralle. 
$260(772)$. E. ciplus, T. Birfnumer.

a. F Europa.

261 (773). E. hoptulana, L. Gorteunumer

a. O' Hannover. G. v. (). Pralle.

b. of Hamover. G. v. Dr. Guthe. ơ Biilge.

$262(774)$. E. caesia, Cretschm.
a. O' Sinyrna.
b. juv. Smyrna.
(. . S Smyrna.
1 Balg.

$263(775)$. E. cia, L. Bippnumuer.

a. Sellweiz.

1 Ballg.

264 (778). E. miliaria, L. (6)rnummer.
a. O' Hammover.
b. O Hammover. G. v. C. Rüst.
c. alb. Isernhagen.
(l. F Hannover. G. v. Dr. Voigt.

265 (783). Plectrophenax nivalis (L.) Edjucenumer.

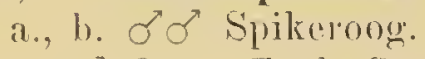

c. of G. v. Zool. Garten.

d. + Spikerong.

e. ㅇ Hannover. G. v. Gr. Münster.

f. \& Hannover. G. v. Gr. Stolberg.

4 Biilge.

266 (784). Calcapius lapponicus (L.) Eporcunumer.

a. o loh. Norden.

b. o Lappland.

Unterfamilie Fringillinae. Fiulau.

267 (7ssa). Ammodromus malimbe, Hartl.

a. Brasilinu. (G. v. D)r. Tälsuer.

$268(788 h)$. A. Iongicaudatus, Gould.

a. Brasilien. (i. v. I)r. Tö̈lsmer.

269 (79) $)$. Haplospiza unicolor (Licht).

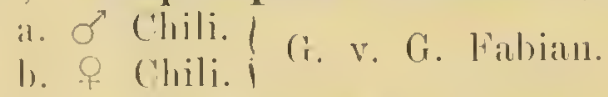

270 (80)-I). Spermophila minnta (Vicill.).

a. Rrasilient.

271 (80) a). Sp. cimmamomea, Bomp.

a. Brasilien. G. v. Mr. 'Tölsmer. 
$27 \cdot 2$ (B)s $)$ Sp. lineola (Lo.).

i. J Brasiliem. (i. v. Mülulmupfordt.

(b. Brilsilien.)

273 (Sog)). Sp. lincata (Gim.).

a. Brisilien.

2ort (912). Plonipara canoxa (Gimel.).

a. ('uha.

275 (\$16). Cardinalis cardiualis (L.) Vioter Sindoimnl.

a. ơ Mexiko.

b. Amprika.

276 (\$16a). Passerina cucullata, Lirht.

a. of Brasilien. G. v. Wresthof.

h. Brasilien. (i. r. l'astor Jordan.

278 (8161). P. dominicana, Prz. Wied.

a. o Brasilin'n.

b. Brasilien. G. v. Dr. Tölsner.

278 (817). Hedymeles ludoviciamus (L.).

a., b. Nordamerika.

(C) J juv. Costarica. G. v. Konsul Nanne.

d. jux. Mexiko.

e. f Costarica. G. v. Konsul Nanne.

279 (819). Guiraca caerulea (L.).

a. $\sigma$ Nordanerika.

b. f Nordamerika.

2 Bälge.

$280(820)$. G. cyanea (L.).

a., b. ơ Südamerika.

․ f Südamerika.

281 (825). Coccothunstes coccothraustes (L.) Siridy= ferubeizer.

a. Of Hannover. G. v. H. Behne.

h. O juv. Hannover. G. v. Kaufmanu Behne.

c. pull. Inonnover. G. v. C. Braunstejn.

d. Hannover'.

282 (82(i). Loxia pityopsittacus, liechst. Sicjerufremz= id)unbel.

a. 8 Denturehland.

h. \& Dentschlanil.

$283(827)$. L. curvilostra (L.) Fidjtenfrejidjunbel.

a. Har\% (i. r. M)r. (intlue.

b. F Har\%. (i. v. I)r. Riiist.

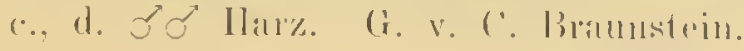


$28+(825)$. L. bifasciata, Brehm.

a. F arl. Archangen.

b. 7 arl. Arhangel.

285 (s.2y). Corythus enurleator (L.) sonfengimper.

a. O Nordeurna. (i. v. Gir. v. d. Sichulenhurg.

b. of Tordeuropia.

i. 7 Nordeuropra.

$286(830)$. Carpodacus erythrinus (Iall.).

a. $Y$ ar. Sibirien.

b. Y ar. Sibirien.

$287(83 \%)$. (. purpureus (Gm.).

a. Nordamerika.

$288(834)$. C. mexicanus (Müll.).

a. ('uha. K. F.

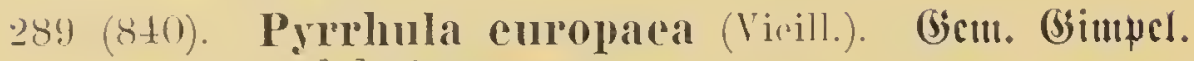

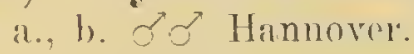

c. ol mol. Hannover. G. v. G. Meyer.

d. \& Hannover. (i. v. C. Braumstein.

e. + Hannover.

290 (851). Seriuns homtulanus (L.) Girlitz.

a. ơ ald. Sürdspanion.

b. q ad. Nüdspanien.

291 (851G). S. camarius X Pyrrhula empopaca. a. Hannover'.

$292(851 \mathrm{H})$. Chr. calduelis y S. canarius.

a. Hannover. G. r. (. Braunstein.

29:3 851k). Pyrluma europaea $\times$ Chrysomituis ealoluelis.

a. Hannover. G. r. Wrinhindler Schulz.

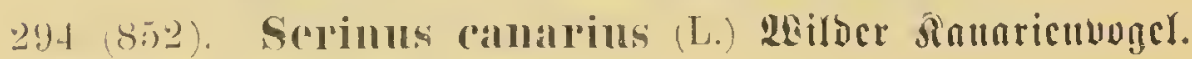

a. Teneriffis.

29.) (852 a). S. (camalius (L.) Bnfuncr Sinunricunogel.

a. $f$ Hanover.

$296(853)$. S. pusillus (l'all.).

a. 7 Lankasus. (i. v. (iötting. Mluseum.

297 (8) (i). Chrysomitris tristis (I

a. કै, b. of jur. Norlamerika.

$2 ! 98(8.59)$. Chro icterion (Licht $)$.

a. of Brasilicn.

299 (86()a). ('hr. Magellanica, Cah.

a. o' C'hili. 
300 (s(5:3). Chr. spinus (1.) Beifig.
i. S" Hanmover.
h. of Hlanmorer. H. r. H. Gidirs.

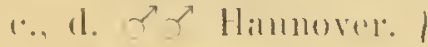
c. L Ilammover.
(i. v. (i. Riiist.

301 (stia). Chr. citrinella (L.) Bitronenfinf.
a. ơ sïddeutsiblindr.
h. \& sï̈ldeutsehland.

302 1866). Carduelis carduelis (L.) Stieglitz.
a. of Itammover.
h., c. Y.t Hannover.
A. juv. Hannover.

303 (868). Acanthis flavipostris (L.) Wergljänffing.

a. O senlesien.
b. o Hannover.

$30 t(869)$. A. linaria (L). Qcinfiuf, Birfenjeifig.

$\left.\begin{array}{l}\text { a. } \sigma \text { jur. Hannover. } \\ \text { b., c. \&o Hannover. }\end{array}\right\}$ G. v. Obereerr. Dommes.

305 (870). A. cannabina (L.) Błnthänf̣ting.
a. $\delta$ Hannover. !
h. O Hannover, G. v. C. Braunstein.

306 (871). Chloris chloris (L.) Briultug.

a. of Hannover. G. v. Op. Pralle.

h., $\because$ o 0 Hannover.

307 (879). Spiza cyanea (L.).

a., b. ఠ" $\sigma$ Nordamerika.

c., d. qf Tittelamerika.

308 (880a). Passerina canora, Cab.

a. of Cuba.

309 (882). (oryphospingus pileatus ('r\%. Wied).

a. Brasilien.

310 (889). Phrygilus unicolar (I) (0.h.).

311 (894). Fringilla coelebs (L.) Budjfinf.
a. ${ }^{\prime}$ Hannovere.
b. ơ Goslar. (i. v. C. Braunstrin.
$\therefore$ F Hamover. G. v. (). Halle.

$312(895)$. F. montifringilla, J. Bergfint.

a., b. ơ (inslar. 1850). (i. v. (¿. Bramstein.

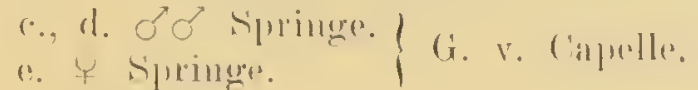

1 balg. 
313 (896). F. nivalis (L.) Educciuf.

a. of sichweiz.

b. Ұ s'rhweiz.

$31+(896 a)$. F. canescens, Griy.

a. Giönland.

$315(897)$. Sycalis flaveola (L.).

a. ơ Brasilipm.

316 (899). Passer nontanus (L.) Febiperfing.

a. o Hannover. (i. v. lithre.

b. F Hammover. (a. v. (). P'ralle.

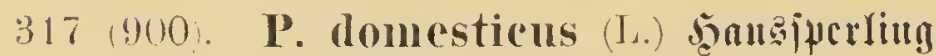

a., h. бo s. alh. Hamnovirl.

( o alh. Hannover. G. v. G. Sichothanber.

d. O Hannovers. (i. v. O. Pralle.

(a) alh. Hamnover.

f. T all, Hanmover. G. v. H. Oppermamm.

g.. . alb. Hamnover.

1 liale.

318 (901). P. domesticus indicus (Jard et silby.).

a. Ostindion. G. v. C. Brannstrin.

319 (911). P. petronius (L.) Etciujpcrfing.

a., h. of sö̈ddentselland.

1 balg.

\section{Familie Ploceidae.}

\section{Unterfamilie Spermestinae.}

320 (915). Amadina ferruginosa (sparm.).

il., b., e. lavil.

1 bale.

321 (918). A. punctulata (L.).

a.. h. Malakkia.

(.) Nalakkiar. G. v. Mülikemptoredt.

d. Naliakile (i. v. Fr. Compril.

1 lialu.

$3202(920) . \quad$ I. castameithorax (inulu.).

i1. Anstralinin.

3023 (925). A. Mlalabarical (L.)

a. Indirill.

304 (927). Spermestes concullata (Swaims).

i., l., (. Mfriki.

I. Nfrika (i. r. Wolpers. 
30.5 (130). Ladda oryzivora (l.) Sicianuger.

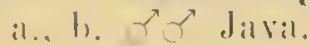

326 (932). Sporothlastes fasciata (iun.) Binudiut.

a. N Nipikir.

b. F Afrikil. (i. v. Mr. Rï̈st.

327 (933). Poëphila suttata (shlitw.).

i. Sustrilinill.

328 (934) P. castanotis (Goulcl.).

a. of Australien. (i. v. Zool. Garten.

1 liale.

329) (937). P. modesta (iould.).

a. A Australim.

330 (940). P. bella (Lath.).

a. Australien.

b. Australien. G. v. Konsul Kauffmann.

331 (950). Estrelda astrild (L.).

a. Südafrika. G. v. Frln. Foten.

h. Südaficik.

332 (951). E. subflava (Vieill.).

a. of Westafrika. G. v. Dr. Voigt.

h. of Westafrika.

c. P Westafrika.

d. Z Westafrika. G. v. Dr. Kohlrausch.

333 (952). E. phoenicotis, Swains.

a.. h. q 7 Afrika.

334 (953). E. cinerea (Vinill.).

a., l., c. $3 \circlearrowleft$ Nordafrika.

d. Nordafrika.

2 l’älge.

335 954) E. temporalis (Latlı.).

a. Anstralinn. (i. v. Konsul Kauffuamm.

1). Anstralinis.

$336(9)(9) \%$. E. allandava (L.).

a., h., (. 3 of Indian.

1. of Indien. (i. v. Froln. Comprerl.

337 (960). Iypochaera nitens nltramanina (im.).

a.. l., r. Westafilika.

d. Westaflikal. (i. r. liowenthal. 
338 (961). Vidua paradisea (L.).

a, b. ठซ Afrika.

c. Of Afrika. (i. v. Rocholl.

339 (962). V. principalis (L.).

a. Of Afrika. Ġ. v. Ir. Mejer.

b. of Afrika.

340 (962a). V. macroura (Gmel.).

a. O Afrika. G. v. Löwenthal.

1. $\sigma$ Afrika. G. v. IMr. Mïhlempfordt.

341. (971). Pyromelana oryx (L.).

a., b. of Süilafrikn. G. v. Dr. Mühlenpfordt.

(. O Sürlafrika. G. v. Götting. Museum.

$342(973)$. P. franciscana (Is.).

a., b. $\sigma^{7} \sigma^{7}$ Afrika. G. v. Löwenthal.

Unterfamilie Ploceinae.

343 (996). Plocens capensis (L.).

a. O' Afrika. G. v. Dr. Mlejer.

b. $\sigma^{\prime}$ Afrika.

344 (1001). P. litteolus (Licht).

a. ơ juv. Afrika.

$345(100+a)$. Nelicmúns baya (Reichb.).

a. Java. G. v. C. Braunstein.

\section{Familie Icteridae.}

346 (1014). Icterns jamaraii (Gmel.).

a. Brasilien. G. v. l'astor Mertens.

1. Brasilien. G. v. G. Breymann.

347 (1015). I. cucullatus, sw.

$348(10) 18)$. I. gionudi, Cass.

a. $\sigma^{T}$ Guatemala.

349 (1019). I. melanocephalus (Wagl.).

i. Mnerika.

350 (1019) it). Ieterus melanopterus (Hartl.).

a. O Columbia. G. v. Zool. Garten.

351 (10.2). I. baltimore (L.).

a., h. $\sigma^{7} \sigma^{7}$ Nordamerika.
a. Nordancrika.

1 Bilg. 


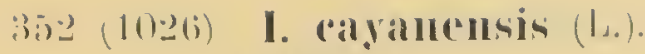

i. M Mrikio.

h. Mrexilio.

(.) Mexiko.

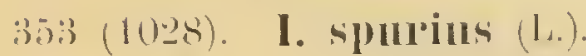

a., b. $\sigma^{7} \sigma^{\prime}$ Nordamerika.

$35+(1029$ a). I. dominicensis (L.).

a. Brisilien.

35:) $(1030)$. Gynnonystax nelanicterus (Vicill.).

a., b. Mexiko.

336 (1035). Agelacus phoeniceus (L.).

a., h., .3 o Noldamerika.

d. f Nordamerika.

357 (1039). Dolichonyx oryzivolus (L.).

a. Noriamerika. G. r. Pastor Mertens.

b., $\bullet$. d. Nordamerika.

358 (1041). Molothus bourariensis (Gm.).

a. J Brasilien. G. v. Pastor Mertens.

a. of linsilien.

1 balg.

359 (1045). Amblyphamphus holosericeus (Scop.).

a. Nordamerika.

360 (1048). Aphobus chopi (Vieill.).

a., h. Brasilien.

2 lialge.

361. (1051). Quiscalus quisculus (L.).

a. Norrlamerika.

$362(1056 \%$ Sturnella magna (L.).

a. h. Nordamerika.

7 biilge.

363 (1058). Trupialis militapis (I.o).

a., เ. of Chili.

(. Chili. G. r. Nolte.

$36 t$ (1066). Cassicus persicus (L.).

a. liasilien.

$365(1068)$ C. haemorrholls (L.).

a., h. Brasilimn.

366 (1068) C. bifasciatus, spix.

i. b. Nurinant. k. li.

c. Mrexilo. (i. v. Komsul Ninme. K. li.

d., r. Mrexike. G. v. Ohrebufgiature Wenrlland. 
367 (10(58b). C'. Cristatus, Cuv.

a., b. Brasilien.

368 (1070). Cassidix oryzivola (Gm.).

a. Mexiko. G. v. Pastor Mertens.

369 (1070a). Pendulinus periporlyyrens, Vieill.

a. Bolivia. G. r. Cohn.

370 (1070h). P. dominicensis, Vieill.

a. Amerika.

\section{Familie Sturnidae.}

371 (1071 a). Lamprotornis insidiator, Cab.

a., b., c. Neu-Guinea.

2 bälge.

372 (1074). Lanuprocolius purpurens (Nüll.).

a., b. of W' Westafrika. G. v. Löwenthal.

373 (1101). Sturuia violatcea (bodd.).

a., b. б o Ustindien. G. v. C. Braunstein.

('. of Ostindien.

374 (1108). Sturnus (Npodiopar) vulgaris (l.) Etar.

a. o Hannover. 1864.
h. O Hannover.

c. o Hannover. 1853.

d. of Hannover.

e. o jur. Hannover. G. v. C. Braunstein.

f. alh. Elze. G. v. Obergerert. Kühnemann.

2. alb. Peinc. 1860. G. r. Siemering.

h. all, Hannover. G. v. O. Stolherg.

i. O Hannover. 1861 . G. v. Rinhold.

k. Hamnover.

375 (1109). S. H1100lor, Temm. Ginfarbiger Etar.

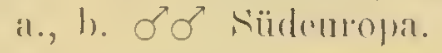

376 (1110). Pastor posens (I.) Mojenjtar.

i. of sïrdentschlind.

b. F sïdderutshland.

1 bialy.

377 (1114a). Acridotheres roistatellus (L.). a., b. Javil.

378 (1117). Gracula religiosa, L.

a. dava.

b. Indien. 
379 (120). Mino dumonti (L, (3...).

i. Nen-Gillincia.

380 (1121). Salrops calvis (L.).

a. of Indien.

381 (1122a). Streptocita toropuata, T'mm.

a. F celebes.

\section{Familie Artamidae.}

382 (1124). Artamus personatus, Gonld.

a. Australien.

383 (112+a). A. cinerens, Virill.

a., h. ơ Anstralian.

$38+(1125)$. A. superciliosus, Gould.

a. of Australien. G. v. Konsul Kanffmann.

385 (1125a). A. leucopygialis, Gould.

a. $\sigma$ Australien.

\section{Familie Dicruridae.}

386 (1143a). Dicrurus carbonarius, Müll.

a., b., c. Neu-Guinea. K. E.

d. O Neu-Guinea.

$387(1143$ b). D. longus, Temm.

a., b. Java.

$388(1143$ \%). D. lencophaeus, Vieill.

a. ${ }^{7}$ Bormeo. G. v. C. Braunstein.

b. Bormen.

\section{Familie Oriolidae. Pixule.}

389 (1145). Oriolus galbula, L. Hirol.

a., b. бo IIannover. G. v. H. Angerstein.

(.) $\sigma$ jur. Hamover.

d. $\sigma^{3} 1877$. Hamnover.

a. juv. Hamnover. (r. v. C. Braunstrin.

f. Of Hamnover. G. v. Lubreseht.

g., Ћ. 2 pull. Hannover. G. v. l'ralle.

i. F Hamnover. 1877.

390 (1147). O. auratus, Virill.

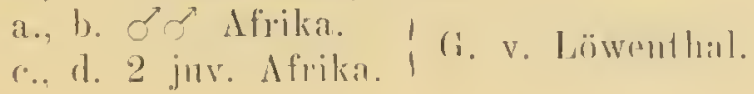


391 (1150). 0. chinensis, L.

a. O Mittelasien. G. v. C. libamnstein.

392 (1152). O. melanocephalus, L.

a. of Indien.

393 (1153). O. larvatus, Licht.

a. O Afrika. G. v. Mr. Mejer.

$394(1156)$. O. xanthonotus, Horsf.

it. of Borneo.

b. O Indient.

395 (1159). O. cruentus (Wagl.).

a. Java.

\section{Familie Paradiseidae.}

396 (1161 a). Glancopis varians, Temm.

a. Borneo.

b. Borneo. K. E.

397 (1164). Ptilorhyuchus holosericeus, Kuhl.

a. O Australien.
b. of Australien.

398 (1165). Ptilorhis magnificus, Vicill.

a. $\sigma^{7}$ Nen-Guinea.

b. ㅇ Neu-Guinea. G. v. Giröner.

399) (1166). Pt. paradiseus, Swains.

a. O Australien.

400 (1167). Selencides niger (Shaw.).

a. of Neu-Guinea.

b. \& Nen-Guinea.

401 (1168). Epimachus specoiosus (Borld.).

a. of Neu-Guinea.

b. \& Nen-Guinea.

402 (1169). Astrapia nigra (Gur.).

a. o Nell- (inimeis.

b. \& Nent-Cininea.

$40: 3$ (1170). Paradisa minor (Shaw.).

a. of Neu-Guinen.

b. If Nell-Guiura.

404 (1171). P. apoda, L.

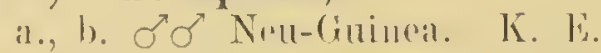

a, d. qf Nen-Gininea. K. li.

405 (1171 i). P. rubla, Virill.

a. 8 Non-Guinea. 
406 (1173a). P. Mngustate Vietoriate, (ail).

i. of Nent-(inillea.

407 (1174). Cicinnmlus pegius (L.) Siümigaparadicanugel.

i. of Nen-(ininuil.

b. + Nen-Guimea.

$108(117 \bar{b})$. Sohlegelia wilsoni (citss.).

it. O Anstralien.

409 (1176). Diphyllodes magnifica (Prm11.).

a. $\sigma$ Nen-Guinca.

410 (1177). Parotia sexpennis (Bord.).

a. of Nen-Guinea.

b. \& Neu-Guinea.

411 (1178). Lophoriua superba (Penn.)

a. of Neu-Guinea.

412 (1180a). Semioptera wallacei, Gray.

a. ơ Batjan.

b. ㅇ Batjan.

413 (1180b). Drepanornis albertisi, Slat.

a. of Neu-Guinea.

414 (118\%). Sericulus mellimus (Lath.).

a. of Australien.

\section{Familie Corvidae. 往alın.}

415 (1183). Pyrphocopax graculus (L.) arpenfrälyc.

a. O’ S'chweiz.

b. ㅇ Schweiz.

+16 (1184). P. alpinus.

a. of Schweiz.

b. Schweiz.

417 (1185). Corcorax australis (Vieill.).

a. Austrilien. G. v. Güner.

418 (1186). Psilorhinus mexicanus, Rï̈pl.

a. Mexiko. G. v. Oherlofwrirtner Wemdland.

b. Mexiko. G. v. Konsul Mä̈lè'.

c. Nexiko.

419 (1.188). Crolenca cyanolenea (Wind.).

a. Brasilien. K. li.

b. Brasilien. G. v. Najor v. Ttsiar.

420 (1190a). Xanthonra gnatimalensis, linp.

a. Guatemala. G. v. Konsm Mällerl. 
421 (1192a). Cyanocorax cyanocapillus, Cah.

a. Mexiko.

$422(1200)$. Cyanocitta cristata (I.).

a. $\sigma$ Nordamerika.

b. of Nordamerika.

c. O Nordamerika / G. v. Zool. Garten.

423 (1201). C. stelleri (Gm.).

i. Californien.

$424(1204)$. Perisoleus infaustus (L.).

a. of Norwegen. G. v. Gr. v. d. Schulenbura.

b. \& Schweden.

425 (1205). Platylophus galericulatus (Cuv.).

a. O Java.

426 (1206). Garrulus glaudarius (L.) (sidflfefler.

a., b. бo $\sigma^{7}$ Hamnover.

427 (1208). G. lanceolatus, Vig.

a. of Hinialaya.

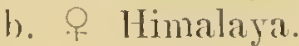

428 (1208a). Cr. californicus Vim.

a. Californien.

429 (1210). Platysmurus lencopterus (Temm.).

a. Ostindien.

430 (1215). Dendrocitta rufa (Scop.).

a. Indien.

431 (1219). D. himalayeusis, Blyth.

a. Indien. G. v. C. Blauustein.

b. Indien. G. v. Götting. Museun.

432 (1224). (yanopolius cooki (15\%).

b., b. viirlspanicen.

433 (1225). Pi(ea pical (L.) Eljter.

a. O Hamover.

b. juv. Hamnover.

c. O Hammover.

t3t (1225a). Pica nuttali, Ant.

a. Californien.

4:3;) (12:6i). Nucifiaga caryocatactes (Brehm) Tnumcu= lyeljer.

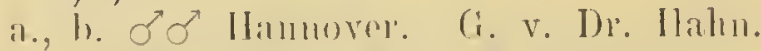

a. of Hatmover. G. v. II. Ermst.

d. of Hatumovor. (i. v. Bieckmatum. 
436 (1228). Colateus monedula (L.) Dofjle.

a. O' Hannover. (i. v. (i. Sorbialtlar.

b. of Hamover. G. v. C. Bramustedn. 1 Bitlg.

437 (1231). Corvus colax, L. Siolfunte.

a., b., (. 3 o Hannover.

d. \& Hamover. G. v, Dr. Hemnerlie.

438 (1241). C. austualis, Gould (comoimides, Vig.).

a. Australien. (i. v. Exc. v. Malorti.

439 (1242). C. corone, L. Sinucufrälye.

a. $\sigma$ Hannover. (t. v. Dr. Vnigt.

b. $\sigma$ Hamover.

440 (1244). C. cornix, L. Miclielfrïge.

a. of Hamnover. (i. v. L. Othenberg.

b. O Hamnover. G. v. O. Pralle.

c. of Hamnover. G. v. W. Voss.

d. \& Hamnover.

441 (1245). C. frugilegus, L. Santfrälje.

a. o Hannover.

b. O Hannover.
c. juv. Göttingen. G. v. C. Braunstein.

d. S Hamover. G. v. O. Wdstr. Wallmamm.

$442(1247)$. Gymnorhiua leuconota, Gray. Grötenuoger.

a. Australien. G. v. Exc. v. Malorti.

443 (1249a). Strepera arguta, Could.

a., b., c. Australien. G. v. Exc. v. Malorti.

1 baly.

$44+(1249$ b). St. anaphonensis, Could.

a. Australien. (x. v. Konsul Kauffmamn.

\section{Familie Laniidae. Hitxinex.}

Unterfamilie Maloconotinae.

445 (1251). Cracticus destructor, Temm.

a. Of Australion.

b. Australion. (i. v. Exce v. Malorti.

446 (1253). Cr. cassicus (Borldl.).

a. Nell-Gininea. 
Unterfamilie Prionopinae.

447 (1283a). Colluriocincla Selbyi, Gmol.

a. Of Australien. G. v. Exc. v. Malorti.

b. of juv. Australien. G. v. Exc. v. Malorti.

\section{Unterfamilie Puchycephalinae.}

448 (1289). Falcumculus froutatus (Lath.).

a. Australien. G. v. G. Gröner.

b. Australien.

449 (1292:). Pachycephala philippinensis, Waldm.

a. Luzon.

$450(1295)$. P. rufiventris (Lath.).

i. of Australien.

451 (1296). P. melanula, (iould.

a. Australien. G. v. G. Gröner.

b. Australien.

\section{Unterfamilie Laniinae.}

452 (1299). Laniellus lencogrammicus, Swains.

a. Java.

c., r. Java. G. v. C. Braunstein.

2 bïlgge.

453 (1300). Corvinella corvina (Slaw.).

a. Westafrika. (i. v. Mr. Mrojer.

$45+(1302)$. Lanius minol, (im. Edfunrzftirniger $\mathfrak{B a i r g e r}$.

a. G Gostar. G. v. C. Bramstein.

45) (130:3). T. excubitor, l. Grojer Bäirger.

i., b., (. 3) o Hammover".

d. jur. Hamnover. (i. ve Dr. Hahn.

a. I Hannover. (i. v. G. Lubrecht.

$456(1320)$. I. senator, 1. Jivtfopfuirger.

ஃ. O Hannover. (i. v. L. Schädtler.

b. of jux. Hannove (Exempl. mit 4 Beinen).

c. of Hamover.

d., 2.2 o juw. Hannover.
f. $q$ juv. Hamnover.

457 (1321 a). L. mepidionalis, Trmm.

a., b. ơ Sürdemropa.

c. ㅇ Südeuropiı. 
the (1:32.2). L. collurio, L. Micmututer.

a. of Hanmover. G. v. C. Bramustein.

b. Of Hammover.

c. P Hannover. G. r. Pralle.

4 Bälger.

459) (1322a). L. spinitorques, Vaill.

a. of Cimton.

460 (1:323). L. unbieus, Licht. Manfentuirger.

a. $\sigma$ Smyrna.

b. jur. Smyrna.

(.) of Smyrnil.

461 (1323a). C. phoenicurus, Gm.

ก. ơ Südsibirien.

\section{Unterfamilie Vireonince.}

462 (1324). Vireo flavifrons, Vieill.

a. Nordamerika.

463 (1333a). Hylophilus cinerascens, Vieill.

a. Surinam. K. E.

464 (1333 b). H. nuelamoxanthus, Licht.

a. o Prasilien.
b. \& Brasilien.

\section{Familie Campephagidae.}

Unterfamilie Campephaginae.

465 (1336). Graucalus nelanops (Lath.).

a. Anstralien. G. r. Konsul Kanffinann.

b., c. Anstralien. G. v. G. Cirönes'

$466(1339)$. (4. Hacii, Less.

a. Indien.

$46 \overline{7}$ (1341). G. papuensis (Gm.).

a. Colebes.

468 (1343). (x. pertoralis, Jard. et Sellhy.

a. Afrika. (i. v. Dr. Mrejer.

$469(1345)$. G. larvatus (s. Müll.).

a. Java. (․ v. Bränstein.

470 (1346). G. temmincki (\$. Müll.).

a. Colebres.

471 (1349) a). Campephaga japlimei, Cionld.

a. Jo Java. 
472 (1349 b.). C. karu, Gould.

a. ㅇ Java.

473 (1358). Pericrocotus miniatus, Temm.

a. Java.

474 (1358 a). P. ignells, Blyth.

a. Indien.

\section{Unterfamilie Ireninae.}

475 (1365). Irena puella (Lath.).

a. O Java.

b. + Java.

Familie Muscicapidae. Flixgunfänñex.

Unterfamilie Iluscicapinae.

476 (1372). Muscicapa grisola, L. (Graucr Flicgen= [đ(])ïuper.

a. 8 Hannover.

b. Hannover. G. v. Dr. Armbrust.

c. Hamover. G. v. Opt. Pralle.

477 (1372a). M. plimbea, Illig.

a. Brasilien.

478 (1373). M. atricapilla, L. Edjwargriafiger Flicgeı= jđ)แลี⿻上er.
a. ơ Hanuover.
\begin{tabular}{l|l} 
a. & J juv. Hammover. \\
c. G Hamover. & G. C. Bramustein.
\end{tabular}
d. \& Hamover. G. v. Dr. Kohlrausch.

479 (1376). M. Darva, Bechist.

a. O Russland.

480 (1:376a). Dasycephala cinerea, Gray.

a. Brasilien.

481 (1382). Poecilodryas supereiliosa (Gould.).

a. Australien. K. E.

482 (1397a). Terpsiphone melanogastra, v. Heugl.

a., b. Ifrikat. (i. v. Dr. Mrejer.

c. Afrika. (i, v. liöwenthal.

483 (1405). Myiagla rubecula (Lath.).

i. O Australinn. 
$48+(1412)$. Sisura inculeta (Lath.).

a. o Australien.

Unterfamilie Ampelinae.

48.) (1434). Ampelis arrulus, L. Eciocujdman.

il., b., 2 o Hannover.

c., d. e., f. $t$ t Hannover. (i. v. C. Branustein.

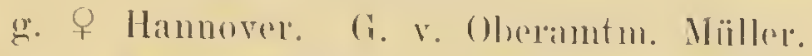

$486(1435) . \quad$ A. cedrorum (Vicill.) catrolincusis.

a., b. Nordamerikia (i. v. I'astor Mlertens.

(. Nordamerika. (i. v. H. bireymann.

487 (1435a). A. japonicus, siels. (phoenicopteress, T'mm.).

a. б Japan.

b. \& Japan.

\section{Familie Hirundinidae. 5rthualtuen.}

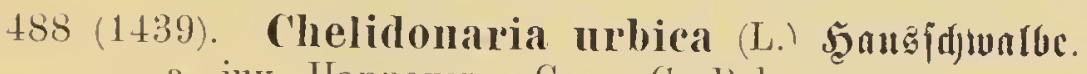

a. jur. Hannorer. G. v. G. Behne.

489 (1440). Clivicola riparia (L.) Itjerjd)wnluc.

a., b., c. 3 \& Hannover. (i. v. C. Braunstein.

490 (1445). Hirundo rustica, L. Timudjatualbc.

a. a!b. Hannover. G. v. Dr. Hundöggerer.

b. $\sigma^{7}$ Hannover.

c. $\sigma^{T}$ alb. Hannover. G. v. C. Braunstein.

d. O Hannover.

e. alb. Hannover. (i. v. Gir. v. Reden.

491 (14t5a). H. flavigastra, Vieill.

a. Brasilien. K. E.

$492(1445$ b). H. tapera, L.

a. Brasilien. K. E.

493 (1+45 c). H. minuta, Pr\%. Wied.

a. O Costarica.

b. Mlexiko.

c. \& Costaricin.

494 (1458). Progne purpurea (L.).

a. of Nordamerika.

495 (1465). Tachycineta albiventris (budd.).

a. Jimsilien. K. E.

496 (1469). Atticora fasciata (Gm.).

a. Siiddamerika. K. li. 


\section{Subordo Oligomyodae.}

\section{Familie Tyrannidae.}

497 (1477a). Taenioptera pyrope, Vieill.

a. Chili. G. v. Fahian.

498 (1484a). Flnvicola comata, Cab.

a. Brasilien. (․ v. 1)r'. 'Tölsner.

499 (1485). Fl. climacura (Vieill.).

a. Brasilien.

500 (1485a). Fl. icterophrys, d'orb.

a. Brasilien. (G. v. I)r. Tölsner.

501 (1485 h). Fl. nengeta, Swains.

i. of Nexiko.

b. . B Brasilien.

502 (1486). Arundinicola lencocephala (L.).

a., b. Brasilien. (i. v. J)r. Tölsner.

(. Surinam.

503 (1492). Copmunts colomus (Vicill.).

a. lirasilien. (G. v. I)r. Tölsner.

2 Bïlete.

504 (1493). Machetornis pixosns (Vieill.).

a. H Brasilien. (i. v. Dr. Tölsner.

$505(1495)$. Centrites niger (Bord.).

a. jur. Surinaul. (i. v. Mlajor v. Uslar.

b. Oo Brasilien. (i. v. Dr. Tölsner.

3 Bïlge.

506 (1496). Platyphuchus mystacens (Vieill.).

a. linasilien.

507 (1499) i1). Todirostrum melanocephalum, L.

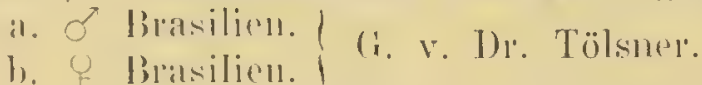

508 (1501: Enscathmus anrifrous, limrm.

a. Brasilien. (i. v. ('oln-Deitelaweig.

$5(1)$ (15)2). Oreluilus anricularis (Vieill.).

a. Brasilirn. (i. v. Mr. Tölsner.

510 (1504). Anacretes parulus (Kittl.).

it. Chili. (i, v. Fabian.

$511,15(18 \mathrm{~s})$. Typannulus albicristatus, Vignols.

a. Brisilinu, K. Li.

b. Basilien. 
5) 12 (1510). Elainea pagana (Lichlt.).

a. Misisilin.

513 (1512). E. cristata, Polz.

i., b. Surinam. K. E.

314 (1512a). E. leneophrys, (ah).

a., b., (. Vranguela. (i. v. Br. 'T"̈̈lsmer.

d. Brasilien. K. F.

1 Bale.

515 (1517a). Pitangus lictore, (imay.

a. Of Mexiko.

b. Brasilien.

c. O' Costarica. (i. v. Konsul Namme.

4 Bälgce.

516 (1521). Megarhyuchus pitangua (L.).

a., b. Brasilien.

517 (1525). Muscivora swainsoui, Pelz.

a. Brasilien.

518 (1526). Pyrocephalus mexicanus, Scl.

a. O Mexiko. G. v. Dr. Tölsner.

วิ19 (1526 a). P. parvirostrois, Gould.

a. of Brasilien.

b. \& Brasilien.

ว20 (1534). Myiarchus tyranmulus, Cassin.

a. Brasilien.

$521(1535)$. MI. ferox (Gm.).

a. Brasilien.

522 (1537). Tyeannus pipiri (Virill.).

a. Nordamerika.

$52: 3$ (1537 a). T. caudifasciatus, d'Orb.

a. $\%$ Antillen.

b. of Antillen.

$52+$ (15:38). T. melancholicus (Vicill.).

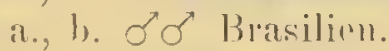
c. q Surinam. K. E.
4 Bälge.

525 (1538a). T. violentus (Viuill.).
a. Brasilirn. (i. v. (C. Brannetrin.
b. Brasilien. 
526 (1539). Milvulus tyramus (L.).

b., b. б $\sigma^{7}$ südamerikia.

4 Bälge.

527 (1540). Oxymynchus flammiceps, Timm.

a. Brasilien.

\section{Familie Pipridae.}

$528(1542)$. Cirrhipipra filicanda (spix.).

a. Brasilien. (․ v. II. Fiedeler.

5.9) (15+4). Pipra aureola (L.).

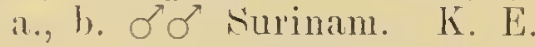

$530(15+7)$. P. auricapilla (Licht.).

a. Brasilien. G. v. 1)r. Tölsner.

1 Balg.

531 (15+8). P. lencocilla, L.

a. Mexiko.

532 (1551). P. Euttulalis, L.

a. + Cayenne.

533 (1551 a). P. anlantialea, Wagl.

a. Cayenne.

534 (1552). Machaeropterus strigillatus, Pr\%. Wied.

a., b. ơ Brasilien.
․ ㅇ Brasilien.

2 Bälge.

535 (1553). Chiboxiphia pareola (L.).

a. ơ Brasilien. (i. v. Dr. Tölsner.

b. of Brasilien.

(๖. juv. Brasilien.

d. ㅇ Brasilien.

2 Bïlge.

$536(1554)$ C. Candata, Shaw.

a. O lirasilien.

2 biilge.

537 (1555). Ilicula militaris (Shaw).

a. of Brasilien.

538 (1556). Chilomathaclis manacus (L.).

a., b. Buasilien.

1 Balg.

539 (1562a). Calyptula clistata, Sw.

a. Brasilien. (i. v. Dr. Tölsner. 


\section{Familie Cotingidae.}

j40 (1571). Pachyrhamphus polychropterus (Vieill.).

a. ร" liasilion.

$5+1$ (1581). Rupicola crocea, Vieill.

a. Ciniana. G. v. Findeler.

$542(1583)$. R. prexuviana, Lath.

a. ơ, h. O Pיru.

543 (1584). Phibaluma flavipostris, Vicill.

a., b. Brasilien.

544 (1588). Cotinga cotinga (L.).

a. O' Brasilien. G. v. Dr. 'Tölsner.

$5+50$ (1590). C. Cayana, L.

a. o Surinam. K. E.

$5+6$ (1592). Xipholena pompadora (L.).

a. O Brasilien. K. E.

b. of Brasilien.

•., d. 2 o juv. Brasilien.

e.. f. 2 juv. Brasilien. G. v. Dr. Tölsmer.

g. Brasilien.

547 (1597). Querula cruenta (Bodd.).

a. ơ Sürlamerika. K. E.

b. 오 Südamerika. K. E.

oł8 (1598). Pyroderus scutatus (Shaw).

a., b., c. Brasilien.

1 Balg.

549 (1599). Cephalopterus ornatus, Geoffr.

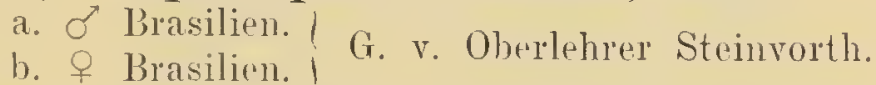

550 (1600). Gymmocephalus calvus (Gm.).

a. Surinam. K. E.

551 (1601). Cymnoderus foetidus (L.).

a. Brasilien.

552 (1602). Chasmorhynchus nudicollis (Vieill.).

a. of Brasilien.

b. \& Brasilien.

\section{Familie Phytotomidae.}

553 (1604). Phytotoma rara, Nolina.

a. of Chili.
b. \& Chili. G. v. Fabian.
1 balg. 


\section{Familie Pittidae.}

554 (1607). Pitta maxima (Müll. et Schleg.).

a. Borneo.

55 (1612). P. atricapilla, Cuv.

a. ơ Sumatra.

\section{Familie Eurylaemidae.}

556 (1622). Eurylaemis javauicus, Horsf. a. Java.

557 (162:3). E. ochromelas, Raffl.

i. Sumatra.

558 (1626). Corydom sumatranus (Rafll.).

a. O' Sumatra.

\section{Subordo Tracheophonae.}

\section{Familie Dendrocolaptidae.}

559 (1629). Loclumias nematura (Sw.).

a. Südamerika.

560 (1646). Philydor rufus (Vieill.).

a. Brasilien. K. F.

ว61 (1650). Xenops genibarbis, Ill.

a. Surinam. K. E.

a. Brasilien. G. v. Dr'. Tölsner.

562 (1656 a). Demdrocolaptes major, Herm.

a. Brasilien.

5633 (1658). Dendrolnis guttata (Licht).

a. Surinam. K. F.

$56+(1659)$. Dendroplex pieus (Gm.).

a. Surinam. K. E.

565 (1661). Picolaptes lencogaster (Swains).

a. Mexiko. K. L. - h. Mexiko.

566 (1661 a). P. albigularis, King.

a. ơ Chili.

b. of juv. Chili. \{ G. v. Fahian.

(․ \& Chili.

56 (1664). Xiphorhyuchus procurvus ('T'emm.).

a. linasilien. 


\section{Familie Formicariidae.}

josis (1667). Thammophilus Ieachi, such.

i. Brasilion.

5(6) (167.4). Th. doliatus (t.).

a. Brasiliens. (i. v. D)r. 'Tölsurer.

h., r. Brasilim.

570 (1675). Th. palliatus (licht).

a. Brasilien. (i. v. Dr. Tälsner.

571 (1675a). Th. Inctuosus, Licht.

a., ๖. Brasilien. G. v. Dr. Tölsner.

$572(1675$ b.) Th. sabinei, Gm.

a. Nfrika. G, v. Dr. Mejer.

573 (1678). Thammomanes caesius (Licht).

a. Jrasilien. (i. v. Dr. Tölsner.

$57 t(1683 a)$. Formicivora gularis, Cab.

a. Brasilien. G. v. Dr. Tölsner.

b. Brasilien.

57. (1683 b). F. intermedia, Cab.

a. ㅇ Brasilien.

5ั76 (1688). Pyriglena lencoptera (Vieill.).

a. O Branilien. G. v. Dr. Tülsner.

577 (1689). Myrmeciza loricata (Licht).

a. Brasilien. Gr. v. Dr. Tölsner.

b. juv. Brasilien.

578 (1691). Hypocnemis lencophrys (Tschr.).

a., b. Brasilien. G. v. Dr. Tölsner.

579 (1694). Formicarius cayennensis (Bodl.).

a., b. Brasilien. (i. v. Dr. Tülsner.

580 (1699 a). Grallaria perspicillata, Law.

a., b. Brasilien. G. v. Dr. Tölsner.

$581(1699 h)$. Fr. Ioricata, siclat.

a. Brasilitn. (i. v. I)r. Tölsner.

Familie Pteroptochidae.

582 (1705a). Hylactes tarni, Sclat.

i. ó chiti.)

b. of Chili. (i. v. Fallian. 


\section{Subordo Pseudooscines,}

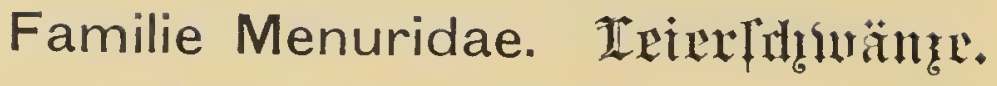
583 (1709). Menura superba, Davies.

a. $\sigma$ Australien.

b. Ơ juv. Australien.

\section{Ordo Strisores (Macrochires),}

Familie Trochilidae. Linlindis.

584 (1709a). Grypus nacvius, Gray.

a. Brasilien.

585 (1710). Glaucis hirsuta (Gm.).

a. Brasilien.

586 (1711). Phaëthornis superciliosus (L.).

a., b., c. Brasilien.

587 (1713). Plı. pygmaelts (Spix.).

a., b. Brasilien.

1 Balg.

588 (1715). Eupetomena maciura (Gm.).

a.--e. Brasilien.

589 (1717). Campylopterus lazulus (Vieill.).

a. Neu-Granada.

590 (1721). Lampornis nigricollis (Vieill.).

a., b., c. Brasilion.

591 (1725). Chalybura buffoni (Less).

a. Columbia.

592 (1727). Polytmus thammanthias (L.).

:., b., (. Brasilien.

593 (1727a). P. linnaei, Gray.

i., b. Brasilien.

$594(1727 b)$. P. furcatus, Giay,

a., b. Guiana.

595 (1728). P. theresiac, Da silva.

a. Amazonas.

596 (1729). Topaza pella (L.).

a. ơ Guiana. K. E.

b. ơ Guiana.

․ \& Guiana. 
597 (1730). Florisuga mellivora (L.).

a. Surunam.

$598(1731)$. F. finsea (1..).

a., b. ช์ ठ์ Brasilier.

c., d. of B Brasilien.

599 (1732). Petasophora thalassina (Swains).

il., b. Mexiko.

600 (1734). P. serrirostris (Vieill.).

a., b. Brasilien.

601 (1736). Angastes superbus (Virill.).

a.--d. Brasilien.

602 (1737). Heliothrix anrita (Gm.).

a. Brasilien. K. E.

b., c. Brasilien.

603 (1739). Chrysolampis moschitus ( $\left.\mathrm{I}_{\text {. }}\right)$.

a., b. ơ Brasilien.

c., d. 우 울 Brasilien.

2 Bälge.

604 (1742). Thalurania glancopis (Gm.).

a. Brasilien.

605 (1749). Clytolaema rubinea (Gm.).

a., b. Brasilien.

606 (1750). Eustepliams galeritus (Molina).

a., b., c. Chili.

607 (1758). Heliangelus clarissae (Longunn.).

a., b. Neu-Granada.

608 (1760). Docimastes ensifer (Boiss.).

a. Brasilien.

609 (1762). Helianthea helianthea (Less.).

a. Ecuadns.

610 (1762 a). H. typica, Gould.

a., b. Nen-Ciranada.

611 (1769). Patagona gigas (Vieill.).

a., b. Amerika.

(612 (177). Rhamphomiceon micporhynchum (Boiss.).

a. liognta.

613 (1788). Lophornis ormatus (Bodd.).

a., b. Brasilien.

614 (1793). Calliphlox amethystina (Gm.).

a.-h. Brasilion.

615 (1797). Calothorax lucifer (Swains.).

a. Mexileo. 
616 (1799). Selasphorus platycercus (Swains.).

a. Nordamerika.

617 (1800). Trochilus colubris (L.).

a., b., c. Pensylvanien.

618 (1800a). Tr. bonapartei, Gr.

a. Neu-Granada.

619 (1801). Leucochloris albicollis (Vieill.).

a. Brasilien. K. E.

b. Brasilien.

620 (1808). Hylocharis sapphirina (Gm.).

a.-e. Brasilien.

1 Balg.

621 (1808a). H. cyanogenys, Gr.

a., b., r. Brasilien.

(i22 (1808h). H. Iatirostris, Muls.

a. Brasilien.

$623\left(1808 r^{\circ}\right)$. H. albirostris, Gr.

i.-d. Brasilien.

3 Bälge.

624 (1810). Chrysoronia oenone (Less.).

a. Trinidad.

62:) (1811). Chlorestes caerulea (Vieill.).

a. Brasilien. K. E.

b. Brasilien.

626 (1815). Panychlora poortmanui (Mnls.).

a., b. Colombia.

\section{Familie Cypselidae. Thathr.}

627 (1817). Collocalia fuciplasa (Thumb.).

a. Jilvit.

(i⿱ 28 (181s). Macropteryx comatus (Temm.).

a. S Sumatra.

(i29) (1820). M. Iongipenuis (Ratin.).

a., h. ơ Jilva.

630 (1823). Chactural pelasegia (l..).

a. Nordamerika.

6:31 (18.4). II emiprocue zomaris (Shaw.).

a. Brasilien.

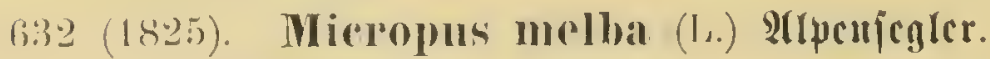

a. Schwri\%. 
6:3:3 (18:6). M. apus (I.) Tururjegler.

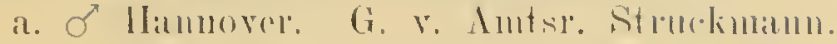

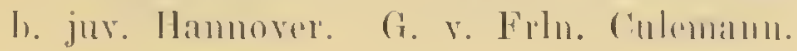

c. Of Hammover.

634 (1831). M. parvos (Licht.).

a. Sundil-Inseln.

\section{Familie Caprimulgidae. Hathtfth̆altur.}

635 (1834). Chordeiles acutipennis (Bodd.).

a. Brasilien, K. E.

636 (1835). Podager nacuuda (Vieill.).

a. $\sigma$ Brasilien.

b. \& Brasilien.

637 (1837). Stenopsis cayenuensis ( $\mathrm{Gm}$.).

a. Ơ Südamerika. Gr. v. Dr. Tülsner.

b. o Sïdamerika.

(‘. O’ Südamerika. | G. v. Konsul Müller.

638 (1839). Antrostomus ocellatus (Tschudi.).

a. Brasilien.

639 (1839 a). A. vociferus, Cass.

a. Ot Amerika.

b. + Amerika.

640 (1839 b). A. Carolinensis, Bonp.

a. of Amerika.

$6+1$ (1843). Scotornis lougicauda (Drap.).

i. Afrikí.

642 (18t4). Hydropsalis furcifera (Vicill.).

a. Brasilien.

$6+3$ (1846). Caprimulgus emropaens, L. Bscur. Mad t= jdjunlbe.

a. Of Hannover. G. v. Siemens.

b. O Hannover.

( o jur. Hannover. G. v. G. Clans.

d. f Hannover. G. v. I'ralle.

e. o Hannover.

644 (1856). C. albogularis (Vig. of Horsf.).

a. Australien.

645 (1862). Nyctibius grandis (im.).

a. Inasilien.

b. Prasilien. (i. v. Tr. Tölsner. 


\section{Familie Podargidae.}

646 (1865). Podargus australis (Steph.).

a. Australien. G. v. Exc. v. Malorti.

b. Australien.

\section{Ordo Picariae.}

\section{Familie Picidae. Thenthte.}

Unterfamilie Picinae.

647 (1868). Picus vipidlis, L. Grrimiperdt.
a. $\delta$ juv. Hannover.
b. of Hamnover.
r. P Hannover.
G. v. Dr. Voigt
d. O Hannover. G. v. Dr. Hahn.

648 (1869). P. viridicamus, Mey. et Wolf. Biraujpedit.

a. of ad. Deutschland. G. v. Dr. Guthe.

b. of juv. Deutschland. G. v. Schädler.

c. O Schöppenstedt. G. v. Tierarzt Samplehe.

649 (1873). P. vittatus, Vieill.

a. $\sigma$ Java.

b. ㅇ Java.

2 우 Bälge.

650 (1873a). P. imperialis, Gonld.

a. Sürlamerikir. G. v. ('. Braunstein.

651 (1874a). P. bicolor, Gimel.

a. of Chili.

652 (1879). Chuysophlegima miniatum (Forst.).

a. Java.

653 (188:3). Colaptes aulatus, L.

a. of Nordamrika. (G. v. Pastor Mertens.

(. P Nordimerika.

654 (1883a). C. aypesi, Andub.

a. O Nordamerikin.

655 (1885). C. pitius (Mol.).

a. Chili.

656 (1887). Dendromus notatus (Licht.).

a. of Nexiko. (A. v. Oherhofgrt. Wendland.

657 (1890a). Chloronerpes ruficeps (Spix.).

a. of Brasiliem. 
65s (1890h). Centurus hypopolins, Min!

a. ơ Mexiko.

b. ot Mixilio.

$659\left(18900^{\circ}\right)$. C. puchelani, Rehb.

a. of Mexiko.

$660(1890 \mathrm{~d}) . \quad$ C. sulfuriventer, Roh.

a. (o) \& Mexiko.

661 (1892). Chloromerpes erythropsis (virill.).

a. of Brasilien.

662 (1892a). Ch. passerinus, L.

a. surinam. K. E.

663 (1894). Chrysoptilus melauochlorus (Gm.).

a. ơ Sïdamerika.

b. \& Südamerika.

1 Balg.

$66+$ (1899). Melanerpes flavifrous (Vieill.).

a., b. Brasilien.

665 (1901). M. carolinus (L.).

a. ㅇ Nordamerika.

666 (1902), M. aurifrous (Wagl.).

a., b. б'ర Nexiko.

c. ơ Mexiko. G. v. Konsul Müller.

d., e. 우 Mexiko.

667 (1902 a). M. Alavigula, Natt.

a. of Nordamerika.

668 (1903). Sphyropicus varius (L.).

a. $\sigma$ Nordamerika.

b., ‘. 웅 Nordamerika.

669 (1905). Dendrocopus pubescens (L.).

a. Nordamerika.

fīo (1906). ID. nuinor (L.) Silciner Buntjued)t.

i. O' Hannover.

fi71 (1907). D. medius (T.) Mittlerer Buntiped)t.

a. O Goslar. G. v. C. Braunstein.

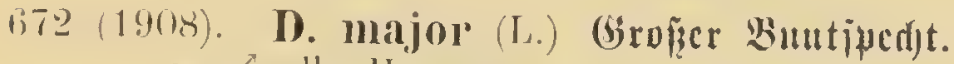

a. $\sigma$ alb. Hannover.

b. of jur. Hamnover.

c. Co Hannover. r. v. (: Brannstrin.

1. \& Hammover.

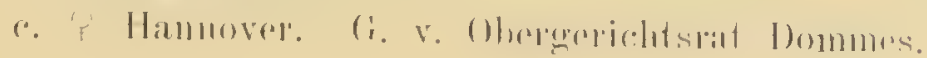


$673(1910)$. D. leuconotus (linelest.) (seriteriped)t.

a. of Deutschlind.

b. of Deutschilimi.

(. O Lappland. (Y. v. Gr. v. d. Schulembure.

6īt (1921). Picoides tridactylus (L.) Drcizeliger Epedit.

i. of Sichweiz.

b. S Sehweiz.

2 (o и. ㅇ) Bälge.

(i75 (19;5). Miglyptes badius, Raffl.

a. Java.

676 (1941). Chrysocolaptes sultaneus (Hodgs.).

a., b. ठक Indien.

․ P Indien.

677 (19)45). Tiga javanensis (Lyng.).

i. Java.

(i78 (1947). Celeus flavescens (Gim.).

a. of Brasilien.

b. of Brasilien. ( G. v. Oherstl. v. Tslar.

r. ot Brasilien.

679 (1948). C. rufus (Gm.).

a. + Gruiana.

$680(1949)$. C. elegaus (Mïll.).

i. Surinam. K. E.

681 (1950). Campephilus pollems (Bp.).

a. of Mexilo. (i. v. Oberlinfugrt. Wendland.

b. \& südamerikil. (i. v. Konsul Namne.

682 (1952). C. robustus (likh1.).

a. O Brasilien.

683 (1953). Ceophloeus limeatus (L.).

a. of Brasilien. K. K.

h., r. 우우 Brasilien.

2 liälge (1 G).

(ist (1955). Thriponax javensis (Howe.).

a. Oo Java.

b. \& Java.

(i85 (195(i). Hemiloplus pulverulentus (Temm.).

a. Java.

(68) (1958). Ipocrantor magellanicus (King).

a. of Patagonion. ( (i. v. Fabian. 


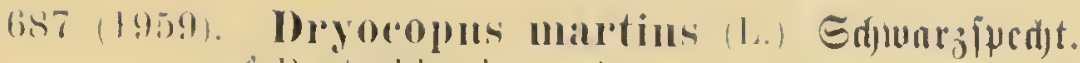

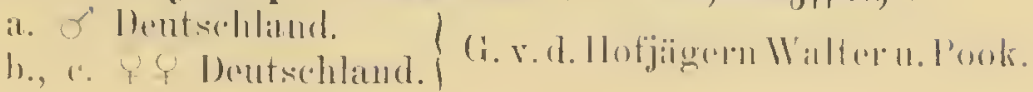

Unterfamilie Picumninae.

(ise (1964). Pichmonus minutus (1..).

i. ơ Brasilicu. K. li.

b. of Brasilian.

« P Brisilien. (i. v. D)r. Tö̈lsner.

? Biilge ơ $\left.\mathrm{n}, y^{\prime}\right)$.

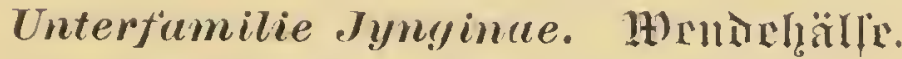

$689(1869)$. Jynx torquilla (L.) Bacmocfjala.

a. O llamnover.

b. Hannover.

\section{Familie Galbulidae.}

690 (1972). Jacamaraleyon tridactyla (Pall.).

a. Brasilien. G. v. Dr. Tölsner.

691 (1974). Galbula albirostris (Lath.).

a., b. Guiana. K. E.

692 (1975). G. rufoviridis, Cab. et Hein.

a. Brasilien. K. E.

b. Krasilien, G. v. Dr. Tölsner'.

c., d. Brasilien.

693 (1976). G. macrura, Vieill.

a. Guiana. kí. li.

\section{Familie Meropidae. Gienentientr.}

694 (1978). Nyctiolnis amicta (Trmm.).

a. Sumatra.

695 (1981). Merops apiaster (L.) Europöijucr Bicuenjreficr.

a. Sïdeuropa. G. v. Frl. v. Pot'n.

b. Sü̈druropa.

696 (1983). M. Marlagascariensis (Briss.).

a. O Marlagaskin.

b. Java.

c. F Marlagaskar.

697 (1985). MI. albicollis, Virill.

a. Afrika. 
698 (1986). M. ornatus, lath.

a., b. Australien. (y. v. Konsul Kauffmamn.

c., d. Australien. (G. v. 1)r. Nü̈ller.

(399) (1988). M. bicolor, Bodd.

a. of Ostindien.

700 (1990). MI. viridis, L.

a. Asien.

701 (1996). Melittophagns lafresnayi (Guérin).

a. Abyssinien.

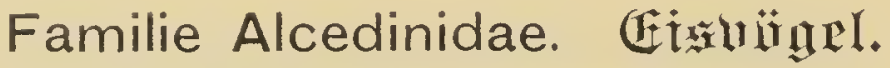

\section{Unterfamilie Daceloninae.}

702 (1999). Dacelo gigas (Bodd.).

a. Anstralien. G. v. Konsul Kanffmann.

b., c. Anstralien.

703 (2002a). Monachaleyon cyanocephala, Brïgg.

a. \& Celebes.

$70+(2002$ h). Dacelo sylvia, Sichleg.

a. Nen-Guinea. (i. v. G. Gröner.

705 (2002 c). D. pileata (S.hleg.).

a. of Ostindien.

b. \& Ostindien.

$706(2002$ d). Cittura sanghierensis, Schleg.

a. Cl Ostindien.

b. \& Ostindien.

707 (200:3). Haleyou colomanda (Lath.).

a. Borneo.

708 (2012). II. senegalensis (L.).

a. Ifrika.

70!) (2015). H. albiventris (sions.).

a. Siidafrika. (i. v. D)r. Mejer.

710 (2017). H. sancta, Viğ. of Horsf.

a. Australien. (i. v. Konsul Kauffinann.

b. Anstralien.

711 (2018). H. chloris (Borda).

i., b. Mblukken. K. li.

( ., d. Molukken.

3) Biilge.

$7120020)$. II. Mareleayi, Jard. et Semby.

a. Anstralien. (i. v. Gröncr. 
$71: 3$ (2026). Coy rufidorsa, strickl.

a., b. Ostindien.

$71+(2028)$. C. tridatetyla (I'all.).

a. Bormo. ki. li.

\section{Unterfamilie Alectininae.}

715 (2037). Pelargopsis melanorhyncha (T'emm.).

a. \& chlobes.

$716(2040)$. Ceryle torquatal (1.).

a. Südamerika. (i. v. Konsul Müllex.

b. Brasilien. G. v. C. Braunstein.

c., d. Brasilien.

$717(2043)$. C. pudis (1.).

a. of Aegryten. G. v. (ir. v. d. Schulenhurg.

$718(20+4)$. C. alcyon $\left(I_{*}\right)$.

a. Nordimerika.

719 (2045). C. amazona (Lath.).

a. of Brasilien.

b., c., d. 3 ㅇ B Brasilien.

1 Balg.

$720(2048)$ C. americana (Gm.).

a. ơ Guiana.

b. \& sürdamerika. K. E.

(־. Guiana.

2 Bälue.

$721(2050)$. Aleyone azurea (Lath.).

a. Australien. (i. v. Konsul Kauffunaum.

7.2.2 (2051). Mleclo ispida (Т.) (sisuogel.

a., b. $\sigma^{7}$ Ilanmover. $^{\circ}$

7.2: (2052). L. ispida bengalensis (Gm.).

a. Java K. li,

Fet (2053). A. semitoryuata, swains.

a. Norrafirika.

725 (20.54a). Alecelo javana, Bodr.

a., b. Java.

$726(205+1)$ ). A. enryzona (Temmo).

a. Java.

727 (2057). Corythomois ryanostigma (liüpr.).

il. Java. 


\section{Familie Bucconidae.}

7.28 (2059a). Bucco chacuru, Virill.

a. Q Brasilien.

729 (206:3). Malacoptila fusca (Gm.).

a. Brasilien.

730 (2063a). M. rubecula, Sclat.

a. Brasilien. K. li.

731 (2065). Momasa morpheus (Hahn).

a. Brasilien. K. K.

b. Brasilien. (i. v. |)r. T'̈̈lsner.

732 (20)(65.). M. flavirostris, Strick1.

i. Sirinam. K. V.

$7333(2065$ h). M. albifuons, spix.

a. Prasilien. (i. v. I)r. Tölsner.

\section{Familie Trogonidae.}

73.4 (20677). Pharomarems paradiseus (Bp.).

a. Ot Vexilio. (i. v. Oberhofgrtu. Wendland.

b. of Costarica.

c. Y Mrxiko. (i. v. ('ohu-I) citelzwign.

735 (2069). Ph. auriceps (fivuld.).

a. of Sïdammerika.

b. \&üdamerika.

$73(;$ (2072). Trogon mexicanus, Swains.

a. Mexiko. (i. v. Istut. Welirsin.

$737(2076)$. T. collaris, Vicill.

a. Brasilinn. (i. v. I)r. T'̈̈lsuer.

b. Iirasilien. (i. v. Colnn-l)ritelzweig.

r., d. Brasilien.

1 Balg.

738 (2077). T. viridis (1..).

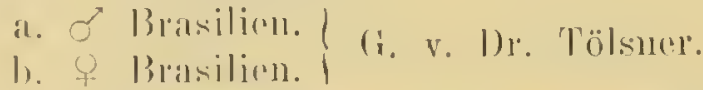

739 (2083). Ilapaloderma narina (Vieill.).

a. O Afrikil. (i. r. Dr. Mejer.

b. I Ahyssinient.

\section{Familie Coraciidae.}

7.10 (2091). Momotas brasiliensis, Lath.

a. Brasilien. K. L.

b., c. Brasilien.

5) Bailente. 
T.11 (og). M. Moficapillus (Vieill.).

a. Mexiku. (i. v. Obortofigertu. Wendland.

2 Biilge.

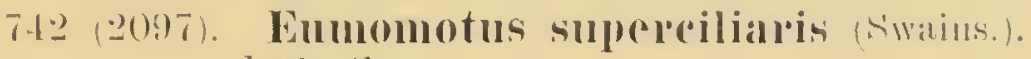

a. of Mrikiko.

h. Mriko.

$7+3(2100)$. Eurystomus orioutalis (1.).

a. Indion. K. L.

b. Indien.

1 Balu.

THt (2101). Colacias garlula, L. Mandelfräbe.

a. O' Hanmorer.

h. Hannover.

7t5 (2101 a). Coraira nudicollis, Vicill.

a. Hirasilien.

\section{Familie Upupidae.}

Tt6 (2109). Upupa epops, l. Bicochonf.

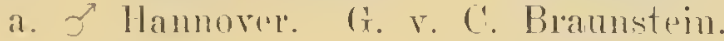

b. of Ilannovere. G. r. Ltut. Schaedtler.

๔. jur. Hamover. (i. v. Major Tellkimpf.

- 7 12111). Irrisor erythrorhynchus (Lath.).

a. Trika. G. v. Rocholl.

b. Ifrika.

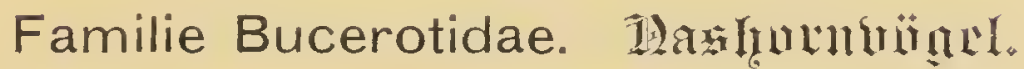

748 (2135). Cranorrhinus cassidix, Termm.

a. (eleloes.

749 (2140). Buceros lunatus, Tromm.

a. Jaria.

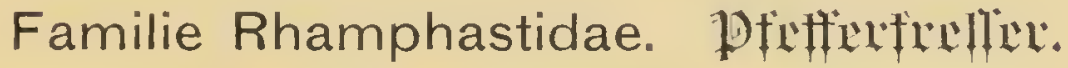

750 (린). Rhamphastus erythorhyndhus (im.).

a., b., c. Brisilim.

751 (2151a). Rh. inca, ciould.

a. Bolivia.

752 (2152). Rh. tocald, Virill.

a. Mrikiko.

753 (2153). Rh. ('alinatus, swains.

a., b. Irexiku. 
754 (215t). Rh. vitelliuns, Licht.

a. Brasilien.

755 (2155). Rh. discolor, I.
a. Brasilien. K. E.
b., c. d. Brasilien. 4 Bälge.

756 (2157). Pteroglossus aracari (L.).
a., b. Brasilien. K. k.
(. Brasilien.

757 (2157a). Pt. azarae, Vieill.

a. Brasilien. (i. v. C. Braunstein.

$758(21571)$. Pt. depbianus, Ciould.

a. Z Mexiko. (i. v. Oherhofgrtn. Wendlind.

759 (2158). Pt. tolouatus, (im.

a., b., c. Vexilo. (i. r. Konsul Nanne.

$760(2160)$. Pt. inscriptus, swains.

a. Südameriki. K. E.

b. Südamerika.

761 (2161). Pt. viridis (L.).

a. Centralannorika.

762 (2163). Sclenidera maculinostris (Licht.).

a., b., c. Brasilien.

763 (2165). S. piperivola (L.).

a. Surinam. K. E.

b. Giliana.

764 (2168). Andigena bailloni (Vieill.).

a. Brasilien.

\section{Familie Capitonidae.}

765 (2175). Pogonorlyuchus bidentatus (Slanw.).

i., b. Afrika. (i. ․ (i. Löwenthal.

766 (ㄴ)1). Megalacma corvina (Temm.).

a., h. Java.

1 Bale.

767 (2) 194 it). M. virens, (iray.

i., b. Java.

1 Pialu.

T6r (2020)). H. versirolor (Rafflo).

a. Borileo.

1 Balug. 
769) (2001). M. javensis (1101sfo).

a. divil.

$770(2004)$. M. almillaris (Temm.).

i. Jilvil.

$771(2210)$. M. losea (Dumont.).

a. Java.

772 (2.210a). M. philippensis, (iray.

ஃ. Indien.

$773(2012$ a). Capito cayennensis, sichleng.

i., b. Ciniana.

\section{Familie Cuculidae. liktkuke.}

Unterfamilie Cuculinae.

774 (2220). Surniculus lugubris (Horsf.).

a. Java. G. v. C. Braunstein.

775 (2221). Chrysococeyx smaragdineus (Swains.).

a. $\sigma$ Afrika.

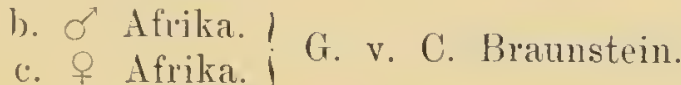

776 (2222). Ch. chrysochlorus (Cab.).

a. Afrika. G. v. Dr. Mejer.

777 (2223). Ch. claasi (Stepl1.).

a. Afrika. G. v. Dr. Mejer.

b. Afrika.

778 (2227). Cacomantis merulinus (Scop.).

a., b. Java.

779 (2229). C. flabelliformis (Lath.).

a. Australien. G. v. Exc. v. Malorti.

780 (2230). Cuculus canorns, 1. (5uropäijd)er Sinfuf.

a. O Catlenburg. 1858. G. v. Antsrat Lüders.

b. ơ Hannover. 1877. (i. v. D)r. Rüst.

c. O' Hannover. 1867 G. v. Oberhofgrt. Wendland.

d. Of Hannover. (i. v. L. Witte.

e. jur. Hannover. 1858 . (r. v. Major Tellkampf.

f. juv. Hannover. 1856 . (r. v. Obererert. Hommes.

(2. juv. Hannover. 1856. (i. v. 1)r. Hahn.

h. F Hannover. (i. v. Dr. v. Holle.

i. of Hannover. 1854.

ot ballig. 
781 (2231). C. indicus, Cab. of Heine.

a. Centralisien.

1 Balg.

782 (2231 a). C. Carolinensis, L.

a. Costarica. G. v. Konsul Nanne.

783 (2234). C. pallidus, lath.

a. Australien.

$784(2234 a)$ C. rubiculus, swains.

a. Afrika. G. v. I)r. Mejer.

785 (2249). Eudynamis punctata (I..).

a., b., c. 3 o sunda-Inselı.

d. of Centralasien.

e. \& Bormeo.

f. ㅇ Sunda-Inseln.

1 Balg.

Unterfamilie Zanclostominae.

786 (2252). Rhinorthra chlorophaca (Raffl.).

a. Sunda-Insehn.

1 Balg.

787 (2253). Piaya miluta (Vieill.).

a. Brasilien.

$788(2254)$. P. cayalla ( ( ...).

a. Guiana. K. Fi.

J). c. Guiana.

j Bïlge.

789) (2257). Rhopodytes viridirostris (Jard.).

a. Madras.

790 (2270). Dromococeyx phasianellus (Spix).

a. Brasilien. (i. v, I)r. Tölsner.

791 (2271). Diploptrous naevius (I.).

a. Brasilien.

792 (2.273). Geococeyx mexicanus (im.).

a. Noxiko. (i. v. latnt. Wehr's.

793 (2276). Neomorphus geoffroyi (Temm.).

a. Brasilien.

$79+(2278)$. Centropus phasianus (Lath.).

a. Australien.

795 (228(1). C. seurgaleusis (L.).

a. Sü̈dafrika.

796 (2283). C. emprercus, Hay.

a. Java G. v. C. Miraminstoin. 


\section{Familie Crotophagidae.}

797 (2087). Guira pirigna (Vieill.).

a. Siirlanerika. (i. v. Rocholl.

798 (20.28). (rotophaga major, L.

a., b. Brisilien.

799) (2289). C. ani, L.

a., h. Brasilien.

4 Bälye.

\section{Familie Musophagidae.}

800 (2296). Corythacola cristata (Vieill.).

a. Afrika.

801 (2301). Corythaix albocristata (Strickl.).

a., b. Afrika.

\section{Ordo Psittaci.}

\section{Familie Psittacidae. Paxagrien.}

\section{Unterfanilie Pioninae.}

802 (2316). Androglossa levaillanti (Gray).

a. Mexiko. G. v. Philippi.

803 (2318). A. ochrocephala (Gm.).

a. Brasilien.

$804(2219)$. A. aestiva (Lath.).

a. O Rrasilien. (;. v. Fr. Sanne.

b. f Brasilien. (i. v. Fr. Insp. Brandmburg.

805 (2320). A. amazonica (t.).

a., b. Brasilien.

806 (2321). A. leucocepliala (1..).

a. Portorico. (i. v. Behne.

b. Cuba, (i, v. Frin, Dromou.

807 (2323). A. ventralis (Müll.).

a. Sit. Jomingo.

808 (2327). A. tarabe (im.).

a. Brasilien. (r. v. Konsul Niemeyer.

809 (2328). A. vittata (Bodd.).

a. I'ortorico. G. v. (Oberilofyet. Wendiand. 
810 (2331). A. anripalliata (lucs.).

a. ó Mittelamerika. (i. v. Mühlenpfordt.

b. Mittelamerika.

811 (2332). A. farimosa (Bodd.).

a. Brasilien. G. v. Konsul Niemeyer.

b. Brasilien.

812 (2337). Pionias senilis (spix.).

a., b. Mexiko. G. v. Oberhofgrtn. Wendland.

(. Costarica. G. v. Konsul Miiller.

813 (2337 a). P. mitıatıs, Finsch.

a., b. $\sigma 0$ Brasilien.

(. ㅇ Brasilien. G. v. Dr. Tölsner.

2 Bälge (1 우).

$814(23+3)$. Urochroma smeda (III.).

a. Brasilen. G. v. Dr. Tölsner.

\section{Unterfamilie Comurinae.}

815 (2:354). Psittacula passerina (1..).

a. of Brasilien. G. v. Zoolog. Garten.

b. of Brasilien.

c. 오 Surinam. K. li.

d., e., f. 3 o ㅇ Brasilien.

2 Bälge.

816 (2359). Brotogerus notata (Bodd.).

a. Brasilien. K. E.

817 (2364). Br. tiriacula (Bodd.).

a. Brasilien. (ir. v. D)r. Nejer.

b. Brasilien. ('. v. Dr'. Tölsner.

c. Brasilin.

818 (2366). Pyrrhuma lencotis (Licht).

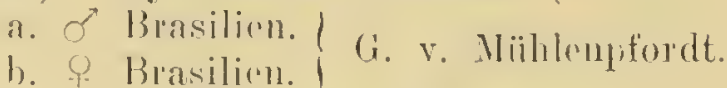

s19 (2372). P. cruenta (['1\%. Wiod.).

a. Brasilien.

820 (2355). Henicognathus leptorhynchus (king.).

a. of Ćlili. (i. v. ('. Braunstein.

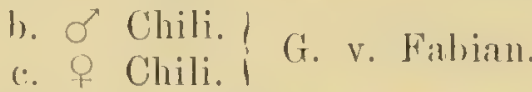

821 (2375). ('omurus petzi (Wingl.).

a. Mexike. (i. v. Oherhofurdu. Wemollande 
8.2) (23)7 a). C. hoftmammi, (ab.

a. Costariea. (i. v. Konsul Namme.

b. Costarica. (i. v. Konsulul Mïller.

s.:3 (2:377h). C. smaragdinus, (iraly.

i., b. Chili.

(r. Chili. (i. v. labian.

824 (20378). C. pertinax (1.).

i. Sïdameriki. K. L.

8.5 (2382). C. carolinensis (L.).

a. Nordamerikin.

$826(2383)$ C. jendaya (im.).

a. Brasilien.

827 (2383a). C. Manday, Desm.

a. of Paramain.

8.28 (2:397). Ala severa (l.) 3wergara.

a. Brasilion.

829 (2398). A. militaris (L.).

a. Mexiko. G. v. Ltut. Wehrsen.

$830(2400)$. A. $\operatorname{macao}$ (L.).

a., b. Brasilien. G. v. Dr. Tölsner.

$831(2402)$. A. ararauna (L.).

a., b. Brasilien. G. v. Dr. Tölsner.

832 (2403). A. hyacinthina (Lath.).

a. Brasilien.

Unterfamilie Psittacinae.

833 (2404). Psittacus erithacus (L.) Braupapagci.

a. $\sigma$ Westafiika.

b. W Westafrika. G. v. Hofjüger Schmidt.

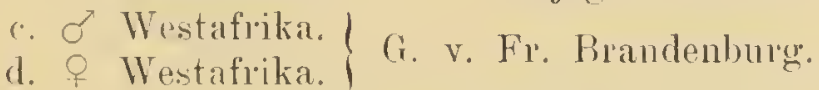

$8: 34$ (2406). Coracopsis vaza (Shaw).

a. Mitdagaskill. G, v. Zoolog. Garten.

Unterfamilie Palaeornithinae.

835 (2408). Agapornis cama (Gm.) Graufüpidjell.

a. of Nadiugaskar.
b. F Nadagaskar. G. v. Frl. Aug. Voss.

$836(2410)$. A. pullaria (L.) ,Insceparable“.

a. O Afrika. (i. v. Zoologe. Mialdert.

b., c. $\sigma^{7} \sigma^{\circ}$ Afriki.

d. 'f Afrikit. 
837 (2412). Psittinus incertus (Shaw.).

a. + Malakka.

838 (2414). Priouiturus flavicans, Cass.

a. 07 Celebes.

839 (2414a). P. discosulus, Bonp.

a. ơ Indien.

840 (2415). Eclectus roratus (Müll.).

a. Molukken. K. E.

b. Borneo.

841 (2416). E. pectoralis (Nüll.).

a., b. Molukken. K. E.

b. Molukken.

842 (2416a). E. rhodops, Gray.

a., b. 우 Borneo. K. E.

c. \& Borneo. K. E.

843 (2416 b). E. Cyanicollis, Müll.

a. ot Indien.

844 (2422). Tanyguatlus luzoneusis (L.).

a. \& luzon.

845 (2422 a). T. burbidgei, Shap.

a. 우 Indien.

846 (2423). Palaeounis longicanda (Bodl.).

a. of Borneo.

b. \& Borneo.

847 (2426). P. fasciatus alexandri (L.).

a. Indien.

848 (2432). P. 1orquatus (Bodd.).

a. juv. Indien. G. v. Gewecke.

Unterfamilie Trichoglossinae.

849) (2437). Coryllis galgulus (L.).

a. ơ Sumda-Inseln.

850 (2437 a). C. bonapartei, Finsch.

a. of Indien.

851 (2448). Domicella rubra (Gim.).

a. Borneo. K. E.

852 (2459). Trichoglossus haematodus (L.).

a., b., c. Australien.

3) Bälge.

853 (2463). T. cyanoglammus, Wagl.

a. Hornco. K. lis. 
sit (2)t7o). T. versicolor, lieg.

a. Australien.

855 (2473). T. placens (Temm.).

a. Australien. K. E.

$856(2476)$. T. pusillus (shaw.).

a. Austration. K, li.

sis (2477). T. concimnus (Nhlaw.).

a., b. Australien. (i. v. Konsul Kauffinam.

r. Australien, G. v. Exe. v. Malorti.

4 Biilge.

s.5 (2478). T. porphyreocephalus (Dietr.).

a. Australim. G. r. Konsul Marwede.

b. Australien. G. v. Dr. Müller.

859 (2478a). T. discolor, Shaw.

a., b., c. Australien.

\section{Unterfamilie Platycercinae.}

860 (2485). Platycercus melanurus (Lear.).

a., b. Australien. G. v. Prof. Müller.

861 (2490). P. amboinensis (L.).

a. O Indien.

862 (2491). P. scapulatus (Bechst.).

a. Australien.

863 (2494). P. spurius (Kuhl.).

a. Australien. G. v. Gröner.

864 (2495). P. zonarius (Shaw.).

a. of Australien. G. v. Zool. Garten.

865 (2497), P. barnardi, Vig. et Horsf.

a., b. Australien.

866 (2498). P. elegans, Gm.

a., b. $\sigma \sigma^{\prime}$ Australien. G. v. Konsul Kauffmann.

(. \& Australien. (i. v. Konsul Narwede.

d. \& Australien.

867 (2501). P. flaviventris (Temm.).

a. o Australien. ( G. v. Lxe. v. Malorti.

c. Australien. G. r. Prof. Mïller.

d. Australien.

868 (2502). P. eximins (shaw.).

a., b. Australien. (i. v. lixe. v. Malorti.

c. Australien. G. v. Dr. Miillar.

1 Baler. 
869 (2503). P. icterotis, Temm.

a. Australien.

870 (2505). D. haematogaster, Gould.

a., b. Anstralien.

871 (2507). P. multicolor (Temm.).

a. of Australien. G. v. Zool. Garten.

872 (2508). P. haematonotus (Gould).

a. Ot Australien. G. v. Zool. Garten.

873 (2508 a). P. hypophonius, Müll. et Schleg. a. Java.

874 (2511a). Euphema elegans, Gould.

a., b. $\sigma^{\circ} \sigma^{\circ}$ Australien.

c. \& Australien.

875 (2516). Melopsittacus undulatus (Shaw.).

a. $\sigma$ Australien. (i. v. Dir. Mülılempfordt.

b. $\sigma$ Australien. G. v. Frau Spangenberg.

c. of Australien.
d. of Australien. G. v. Frau von Berndorf.

e. 우 Australien.

Unterfamilie Stringopinae.

876 (2518). Stringops habroptilus, Griy.

a. Neuseeland.

\section{Familie Cacatuidae.}

877 (2524). Calyptorhyuchus banksi (Lath.).

a. Australien.

878 (2527). C. funereus (Shaw.).

a. Australien. G. v. Gröner.

b. Australien.

879) (2528). C. galeatus (hath.).

a., b. Australien. (i. v. Dr. Müller.

$880(2529)$. Cacatua tritou, Temm.

a. Anstralien. (i. v. Stumke.

$881(2530)$. C. galerita (Lath.).

a. Anstralien. G. v. Lixe. v. Malorti.

b. Anstralien. (i. v. Gröner.

$882(2537)$. C. poseicapilla (Vieill.).

a. Anstralinn. (i. v. Zool. Garten.

8.83 (25)38). (. sanguinea goffini (Finsch.).

ஃ. Molukkin (?). (i. v. Zoonl. (iantent. 
Sist (20)60). Licmetis nasiens ('T'mm.).

a. Australien. (i. v. Konsul Kauffimanu.

b. Australiens. (i. v. Zool. Ciarten.

\section{Ordo Raptatores, Hanubüinel.}

\section{Familie Strigidae. Giftru.}

Unterfamilie Striginae.

S85 (2541). Strix flammea (L.) Edjlcicrutle.

a. ó Hamnover. 1862 .

b. of juv. Ifamover. 1858 . G. v. Baumyarelt.

$\therefore$ \& Himmover. 1863 .

$886(2542)$. St. flammea perlata (Licht.).

i., b., (•. Nordamerika.

$887(2545)$. St. castanops, ciould.

a. Anstralien.

\section{Unterfamilie Syrniinae.}

888 (2548). Speotyto cunicularia (Mol.).

a. Amerika.

889 (2549). Ninox maculata (Vig. et Horsf.).

a. Australien. G. v. lixe. v. Malortie.

890 (2556). Glancidium noctua (Retz.).

a. O' juv. Hamover. Gr. v. G. Rüst.

b. Deutschland. G. v. Frau Castellan Meyer.

(. O ad. Hammover. G. v. Braunstein jun.

d. Dentserhland.

891 (2556 a). Gl. ferrugineum, Cuv.

a.. b. Brasilien.

$892(2556 h)$. Gl. namum, King.

a. O St. Jagno. (i. v. Konsul Müller.

899 (25)6(c). Gl. minutissimum, schleg.

a., b. Brasilien.

894 (2556(1). Gl. nudipes, Less.

a., b. Afrika.

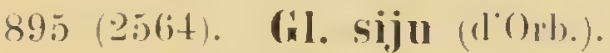

a. ơ Cuba.

b. \& Cuba. 
$896(2565)$ G1. passeriumm (1.) Eperliugäenle.

a. Z Europa.

897 (2556). Nyctala tengualuni (Gm.) Sianbufufaud.

a., b. Europa.

$898(2567) . \quad$ N. acadica (Gm.).

a. Nordamerika. G. r. Rat Dressol.

899 (2568). Surnia ulula (1.) Eperbereule.

a., b. Nordameriki.

(․ Luropa.

900 (2569). Nyetea scandiaca (L.) Edjucrenle.

a. O Nördl. Halbkgl. (i. r. Zoolog. Garten.

b. ơ Nördl. Halbkgl.

(. O Nördl. Halbkel.

901 (2570). Syrnimm aluco (L.) Walsfand.

a. of Hannover.

b. $\sigma$ jur. Hatmover.

(.) Hammover. (i. v. Forstdir. Burekhardt.

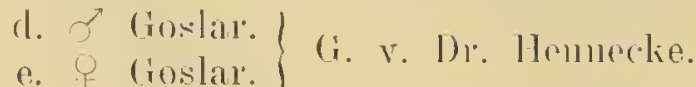

f. O llammover. G. v. 11. Lubrerht.

902 (2571). S. mralense (Pall.) Ilraleule.

a. Nordosteuropa.

903 (2572). S. lapponium (Retz.) Lapplandencule.

a. \& Lappliand.

904 (2573). S. nebulosum (Forst.).

a. $\sigma$ Nordamerika.

b. O Nordamerilia.

905 (2574). S. hylophilum (Trmm.).

a. Sürlamerikit.

$906(2576)$. S. perspicillatum (Lath.).

ஃ. Brasilien. (i. v. J)r. Tölsmer.

907 (2576a). S. woodforti (130mp.).

a. Afrikia. G. v. II. Rochloll.

908 (2578). S. hulumlum (D)and.).

a. Rrasilien. (i. r. I)r. Tö̈lsuer.

Unterfamilie Buboninae.

909) (2582). Pisolhina lencotis (T'emm.).

a. Alyssinien.

9) 10 (2587). P. scops (1.) Buergulgreule.

a. Oे Europa.

h. \& Europa. 
911 (2)

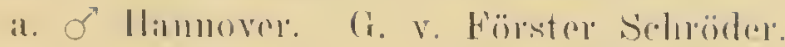
b. Y llamover. (i. ve (iastwilt Rellse.
(i) to Ilamorover.
1. Of jux. Hammover.

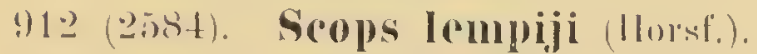

a. Q Ostinclien.

913 (258sb). Seops aldrovandi, Will.

a. Of Nordinmerikit.

b. Ir Nordamerikia.

914 (2590). Asio accipitrinus (Pall.) Sumpfolyreule.

a., h., ‘. 3 o Hamover.

d., e. 2 \& Hamover.

$915(2592)$. A. stygins (Wagl.).

a. Cuba.

916 (2593). Bubo bubo (L.) Itfu.

a., b., c. Europa.

d. Wolga-Gehiet. G. v. Gr. v. d. Schulenburg.

917 (2594). B. virginianus (Gm.).

a. Amerika.

918 (2596). B. bengalensis (Frankl.).

a. Hindostan.

919 (2599). B. lacteus (Temm.).

a. Afrikia.

920 (2604). Ketupa ceylonensis (Gm.).

a. Bengalen.

921 (2605). K. ketupa (Horsf.).

a., b. Suncla-Insehn.

c. Sunda-Inseln. (i. v. C. Braunstein.

\section{Familie Falconidae. $¥ a l k m$.}

\section{Unterfamilie Falconinae.}

922 (2606). Falco islandus (candirans), Brïnn. Jagdăff.

a., b., r. ;) of arl. Grömintml.

dl. ơ juv. (irönlandl.

(2. juv. Giönland.

f., g. 2 q o cirionlandl.

h.. i., k. 3 ? juv. Grönland. 
$923(2607)$. F. lanarius, L. $\mathfrak{B}$ iurgfalf.
a. of Banat.
b. (ठ) \& Banat.
r. Q Wolga.

924 (2608). F. peregrimus, Tumst. QBanderĩnlf.
a. of Hannover. 1865 .
b. of IIannover.
r. of Hamnover. (i. v. Dr. Müller.
d. Of ard. Fuhrbergen. G v. Hofjager Ponk.
a. of Deinsen b. Hannover. 1859.
f. Y jur. Hamnover. (G. v. Zoolog. Garten.
g. O juv. Hannover.

925 (2610). F. tanypterus, Schleg. (biamicus).

a. Abyssinien.

9.26 (2611). F. subbuteo, Т. Banuralf.

a. O Sehude b. Hamover. (i. r. H. O. Stolberg.

b. of Hammover. 1858 . G. v. Dr. Armbrust.

‘. of Ilammover. (i. v. (.. Bramstein.

d., e. ơ Hannover.

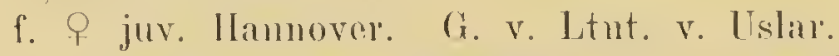

g. P Steinwedel b. Hamover. G. v. H. Nobbe.

1 Balg.

927 (2612). F. eleonorae, Gim.

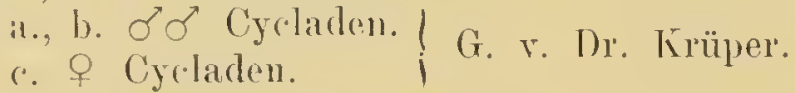

928 (2617). F. aesalon, Tunst.

a. of Hamuver. G. v. Brandt.

b. $\sigma$ juv. Hamnover.

(. $\sigma$ juv. Hamnover. 1861 . G. v. Pape.

d. of juv. Hammover. G. v. C. Riust.

e. P Hildesheim. G. v. Pralle.

f. of Hamover.

우 Balg.

$929(2619$ a). F. femoralis, T(1mm.

a., b. Brasilien.

$930(2620)$. Cerchneis timunculus (L.) Turmfalf.

a., b. бo llannover.

(. of Hannover. (i. v. limbure.

d. of juv. (irstar. G. v. D)r. Hennecke.

(2. Of juv. Ilamover.

f. O Hannover. (i. v. C. Biraunstrin.

«. O juv. Algermissen.

3) Biiluge (2 $q)$. 


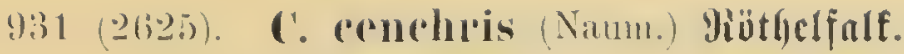

a. ơ Griechenland.

b. pull. Crrierhembund.

e. 7 Griechenlanol.

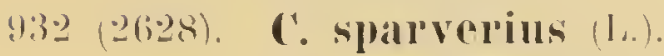

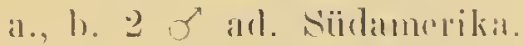

(., of jur. Sü̈lamrerika.

d. of jus. Südamorika. (i. v. Dir. Mertens.

a. Z Irxiko. G. r. Oberhofurtu. Wrendland.

f. F Amerika. (i. v. C. Bramstein.

g. O jur. Sürlamerika.

h. i. St. Thomas. G. r. A. Eihler's.

+ Büluge.

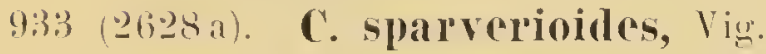

a. of Mexiko.

b. \& Mexiko.

9:34 (2029). C. vespertiuus (L.) giotijujarfé.

a. Ơ Sïdrussland.

b. S sïnlussland.

935 (2631). Microhierax caerulescens (1.).

a., b. Sumatra.

1 Balg.

936 (2634). Hieracidea berigora (Vig. et Horsf.).

a. Tasmania. G. v. Dr. Mü̈ller.

b. Tasmania.

\section{Unterfamilie Polyborinre.}

937 (2636). Ibycter americanns (Bodd.).

a. Brasilien.

938 (2641). Polyborus cheriway (Jac4.).

a. Mrxiko. (i. r. Konsul Nüller.

b. Mexiko.

939 (2642). P. tharus (Nol.).

a. Prasilien. (i. v. Konsul Namme. K. li.

b. Brasilien.

Unterfamilie Gypaëtinae.

940 (2644). Gypä̈tus barbatus (1.) \&ämmergeicr. a. Shllweiz. 


\section{Familie Aquilidae. Holer.}

Unterfamilie Pandioninae. Fifthatlex.

941 (2646). Pandion haliacitus (L.) Fifdadoler.

a. $\delta$ Hannover. (i. v. Oberhofjäger v. Reden.

b. \& Catlenburg. G. v. Amtmann Lueders.

$942(26+6 a)$. P. leucocephalus, (iould.

a. Australien.

\section{Unterfamilie Buteoninae.}

\section{Gruppe A. Milvinae.}

94:3 (2651). Pelnis apivorus, ᄂ. Bejpcubujiard.

a. of Hannover.

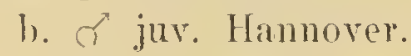

(. F Dorsterlt, Hannover.

d. Of Hannover.

$9+4$ (2652). P. ptilorhynchus (Temm.).
a. of Borneo. K. L.
b. 을 Bormeo. K. E.
$\therefore$ \& juv. Borneo. K. L.

945 (2654). Regerhinus uncinatus (Temm.).
a. jur. Brasilien. K. L.
b. Brasilien.

$946(265 \overline{7})$. Ietinia plumbea (im.).

a., b. Brasilien. (i. v. I)r. Tölsner.

947 (2662). Elanus leucurus (Vicill.).

a. jux. l'ort Natal.

b. Brasilian. (i. r. Ciröner.

c. ('hili. (r. v. Hennecke.

1 Bale.

$948(2664)$. Elanoides furcatus (L.).

i., b. Amerika.

949 (2665). Milvus milvus, (h.) Moter Gindelueil), Milnu.

a. of ad. Hammover.

b. of Deinsen b. Hannover.

(๖. J juv. Hamnover. G. v. r. Hagen.

d. of Gostar. \{ (i. v. Hemnecke.

f. Otur. Gostar. (i. v. (C. Braunstein.

g. O Deinsen b. Hannover. 


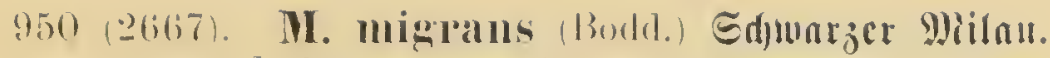
i. of Vorpommenem.
b. Y) Vorponumern.
(i. v. 1)r. Riiist.
(‘. + vïdlossland.

951 (2668). Haliastur indus (Hokld.).

i. Indien.

\section{Gruppe B. Buteoninae.}

952 (2673). Upubitinga nubitinga (Gin.).

a. Brasilion.

95:) (2675). Lencopternis lacernulata (Temm.).

a. Brasilien.

954 (26) Gi). Gevanoaitus melanoleneus (Vieill.).

a. O Nlittelamerika.
b. jur. Nittelamerika.
(.) O Nittelamerika. G. v. Hüttenmeistor Nolte.

955 (2677). Asturina nitida (Lath.).

a. Brasilien. (․ v. Dr. Tölsner.

956 (2675a). A. Iongicauda, Less.

a. Brasilien.

957 (2678). Rupornis nattereri (Scl. et Salv.).

a. J Mexiko. K. E.

1. F Nlexiko. K. F.

c. jur Mexiko.

d. $d$ Mexiko.

2 biälye.

958 (2685). Buteo ferox (imi.).

a. O Sïdrussiant.

1). \& sürlrussland.

959 (26866). B. buteo (โ.) Min̈uichujiard.

a. O Hannover. G. v. Major Tellkampf.

b. o Hannoves.

( C) Hannover. (i. v. (') Braunstein.

(l. of Deinsen h. Hannorer.

P. I)einsen b. Hannover.

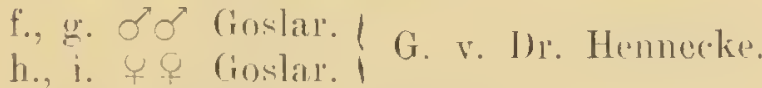

k. juv. Benthe b. Ilannover.

l. juv. Ilamosere.

111. F II:Hmover.

\} (x. v. ('. Bimunstein.

n. F Hannover. 18633 (i. v. Infeifor.

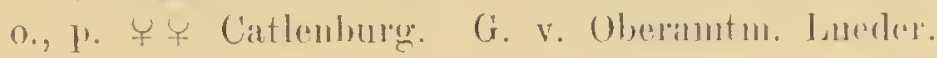


1. + Hamnover. 1882. G. v. Major Lïder.

l. of Achim b. Bremen. G. v. Landrat v. d. Decken.

s. f Lauterberg.

2 ๙ Bälgee. 3 ㅇ Bälge.

960 (2687). B. desertorum (Daud.).

a. of Sürlıussland.

b., c. Südrussland.

961 (2691). B. borealis (Gim.).

a. Nexiko. G. v. Konsul Müller.

$962(2693)$. B. lineatus (Gm.).

a., b. $\sigma^{\top} \sigma^{\top}$ Nordamerika.

(. f Nordamerika.

1 Balg.

963 (269:3). B. polyosoma, Less.

a. Sürlamerika. G. v. Ilüttemmeister Nolte.

$96+(2693 \mathrm{~b})$. B. albicollis, Gray.

a. O Suidamerika.

965 (2695). Archibuteo Iagopus (Brïn.) Sinulyujunjiard.

a. $\sigma$ Hannover. G. v. Inx. Voigt.

b. O Hamnover. (i. v. v. Stolzenberge.

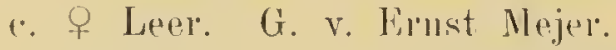

d. \& Goslar. G. v. I)r. Hennecke.

e. - Hannover. G. v. Poock.

f Balg. ơ Balg.

\section{Gruppe C. Haliaëtinae.}

966 (2698). Haliä̈tus albicilla (L.) Secabler.

a. $\sigma$ Wangeroog.

b. б juv. Limopa.

(.) ad. Wangeroog. G. v. Capt. Ehel.

d. Of Biemen. (i. v. Mr. Kohlrauseh.

(.) Hoja.

$967(2702)$. H. Iencogaster (Gim.).

ஃ. Y Indien.

\section{Gruppe D. Aquilinae.}

968 (27(9)). Uroaitus audax (Lath.).

a. Australien. (i. v. Konsul Marwede.

969 (2705). Aquila cheysä̈tus (L.) Stciunder, Bbuldoler.

a. $\delta$ Nienhnum.

b. Fuhberg, Hammover. G. v. Hofjäger lewecke.

c. Tö̈lisde, Hammover. 
960) (2706i). I. melanäitus (1.) Sinijernoler.

a. Oे Banat.

b. Oे jur. Simrepta.

(r. jur. Luropal.

d. of Banat.

971 (2711). A. Clamga, l'all. Grufger Edjreinder.

a. O" Mittlere Wolyar.

b. Ot südrusstand.

c. ơ sarepta.

1., e. 5 o juv. Siarepta.

f. Neuhaus, Hamnover. G. v. Leihjiager Tegtmeyer. g., h. 2 o sarepta.

972 (2720). Spizätus tyramus (Wied.).

a. of lirasilien. (i. v. I)r. Tölsner.

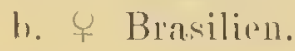

973 (2720a). Sp. ornatus, Vieill.

a. of Brisilien.

b. of jur. Brasilien.

c. \& Brasilien. G. v. H. Niemeyer.

d. f jur. Brasilien. G. v. Hauptmann Niemeyer.

e. Y Mexiko. G. v. Oherhofgrtm. Wendland.

$97 t(2720$ b). Sp. caepulescens (०).

a. Sunda-Inseln.

Gruppe E. Thrasaëtinae.

975 (2722). Morphnus guianeusis (Daud.).

a. Brasilien. G. v. Dr. Tölsner.

\section{Gruppe F. Accipitrinae.}

976 (2723). Accipiter velox (Wils.).

a. Nittelamerika.

977 (2724). A. nisus (L.) Eperber.

a. O' ad. Hannover. G. v. Hugro.

h., c. 2 o jur. Hannover.

d. $\sigma$ ad. Hamnover.

e. jo jur. Hannover. G. v. C. Brannstein.

f. $\sigma$ ad. Hannover. G. v. Forstarlo. Sievers.

g. $\sigma^{7}$ ad. Hannover. G. v. \%oolow. Garten.

h. $\delta$ ard. Hannover.

i. O' ard. Hamnorer. (i. v. Jïger Weber.

j. $\sigma^{7}$ jux. Hamover. (i. v. O. G. R. v. Werllof.

k. O' ad. Hamnover. G. v. Oherjagdmstr. v. Reden. 
l., 111. 2 o juv. Hannover.

11., o., p. 우우우 ard. Hamnover.

4 Bälge.

978 (2724a). A. xanthothorax, Less.

a. of Brasilien.

b. 우 Brasilien.

$979(2724 b) . \quad$ A. fringilloides, Vig.

a. of Cuba.

b. 우 cuba.

980 (2730). Astur approximans, Vig. et Horsf.

a. O Australien. K. li.

b. O Australien. K. F.

c. Australien. G. v. Oberhofmarsehall Fixe. v. Malorti.

981 (2731). A. tachiro unduliventer (Rüpp.).

a., b. Afrika.

982 (2731 a). A. rayi, Vig. et Horsf.

a., b. Australieur.

983 (2738). A. novaehollandiae (Gm.).
i. of Australien.
b. o Anstralien.
G. v. Exre, v. Malorti.
(•. jur. Australien.

984 (2739). A. palumbarius (L.) Şiifuerfalbidt.
a. O Hannover.
b. of Catlenbure. (i. v. Oberamtur. Lueder.
r. of Wathlingen, Hannover. G. v. Gorling.

1. $\delta$ jur. Goslar. (t. v. Dr. Hemmereke.

ค. $\sigma$ jur. Hannover. (i. v. Oherjagdunstr. v. Reden.

f. of juv. Bordenau. I G. v. Rentier Bartels.

g., h. 2 of juv. Bordenau.

i. 委 ad. Ilannover. G. v. Othenberg.

j. Q Hannover. (i. r. Gir. v. Bernstorff.

k. S Hamnover.

l. O Ilammover. (i. v. C. Braunstein.

m. O Hammorer. G. r. Goedekemeyer.

3) Bialge.

$985(2750)$. Circus macrurus (Gm.) Stcppeuncige.

i. of Hannover. (r. v. I)r. Voigt.

h. of jur. Inamover. G. v. Letut. Stollorere.

r. O Borkum. G. v. (ir). Anfseher lingellated.

1. of Wolga.

e. F Goslar. G. v. C. Briansterin. 
986 (2751). C. Cyaneus (I.) Siormucifje.

it. O IIamover. G. v. Major Trollkampfe.

b., c. ơ o sarepta.

1. $\sigma$ lï̈beck.

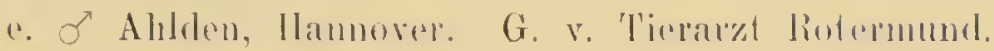

f. of jur. Hamnover.

u. O Hanmover.

I. O Hannover. G. v. Dr. Hahn.

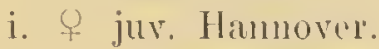

987 (2752). C. pyandous (L.) Baicjenueilje.

a. O์ Hannover.

b. $\sigma$ jur. Hannover. G, v. Feldjüger Schroeder.

(c. $\delta$ juv. Hagenburg.

d.- g. 4 jur. Hannover.

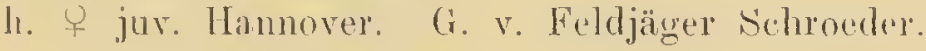

i.-m. 5) pull. Hannover.

11., o. \& \& Hannover.

p.-.s. 4 pull. Hannover.

t. \& Hannover.

u. pull. Hannover.

v., w. to Hammover.

2 \& Bälye.

988 (2755). C. approximans, Peale: (assimilis).

a. Australien. (ì, v. Dr. Müller'.

989 (2759). C. aeruginosus (L.) Mioljrucifye.

a. of lolmatien.

b. q jux. Deinsen b. Hannover. G. v. Pralle.

990 (2759 a). (. macropterus, Vieill.

a. Brasilien. (i. v. I)r. Hennerke.

Gruppe G. Circaëtinae.

991 (2761). Helotarsus ecaudatus (Uaud.) Binufelnorer.

a. Afrika.

992 (2763). Spilornis bacha (Maud.).

a. Java.

993 (2768). Circäitus gallicus (Gm.) Erffnugcunder.

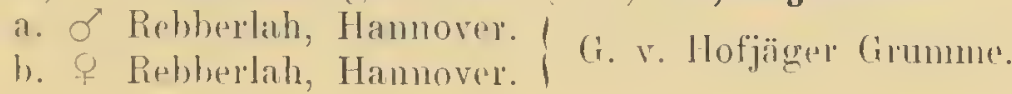

Familie Vulturidae. (filux.

994 (2777). Gyps kolbii (l)aud.).

a. Sürlafrika. 
995 (2778). G. fulvus (Gm.) (Siüujegeicr.

a. Ungarn. G. v. Lionsul Marwede.

996 (2781). Vultur monachus, L. Sinttengcier.

a. of Dalmatien.

\section{Familie Sarcoramphidae.}

997 (2783). Catharista aura (L.).

a. Mexiko.

998 (2784). C. atrata (Bartr.).

a., b. Brasilien. K. E.

999 (2785). Sarcorhamphus papa (L.) Röuigegeicr.

i. of Brasilien.

b. of Brasilien. (i. v. Oheramtm. Nanne.

c. \& Brasilien. G. v. Zoolog. Garten.

\section{Ordo Gyrantes, Girrü̈gl.}

\section{Familie Columbidae Taulen.}

1000 (2787). Oena capensis (L.).

a. Westafrika.

1001 (2788). Geopelia striata (1..).

a. Sundr - Inseln.

1002 (2792a). Macropygia macrura, Gray.

a. Java.

1003 (2794). M. ruficeps ('T'emm.).

a., b. Borneo. K. k.

(. Borneo. (G. v. D)r. Müller.

1004 (2795a). Turacoena reinwardtii, Temm.

a. o Bormeo. ki. Li.

1005 (2796). Ectopistes migratorius (L.) Eandortanbe.

i. Tordamerika. (i. v. C. Braunstein.

$1006(2797)$. Columba palumbus (L.) Hitugeltauluc.

a. Ơ Hannover.

b. of Hannover. G. v. H. Behne.

( $\cdot$.

$1007(2798)$. C. oenas, 1. Şobltaulde.

i. o Hamnover.

b. \& Hannover. 
$1008(2799)$. C. livia, T. Jeljentmule.

a. of sülffrankreich.

b. \& siidfrankrerich.

1009 (28)si). C. aldpuatrix, Timm.

a. Afrika. G. v. Koolog. Gartens.

$1010(2810)$. C. arancana, less.

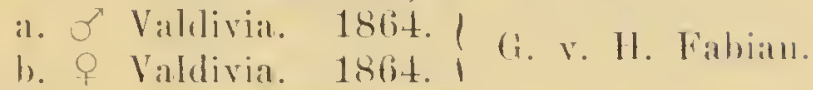

1011 (2813). C. rufina, T'mm.

a. Surinam. K. F.

1012 (2814). Scardafella squamosa ('Temm.).

a. Brasilien.

1013 (2815). Chamaepelia passerina (L.).

a., b. Surinam. K. k.

(. Brasilien. (่. v. Mr. Tölsner.

d. Mexikn. G. v. Oberhofgrtn. Wendland. 1 Balg.

$101+(2817)$. Ch. talpacoti (Temm.).

a. Südamerika. K. E.

1015 (2823). Peristera cinerea (Temm.).

a. of Brasilien.

b. o Brasilien.

1016 (2824). Leptoptila rufaxilla (Rich, et Berm.).

a. Surinam. K. E.

1017 (2828). Chalcopelia afra (L.).

a. O Afrika. G. v. Zoolog. Garten.

1018 (2829). Turtur rupicolıs (Pall.).

a. Japan. G. v. C. Braunstein.

1019 (2830). T. turtur (L.) Turteltaulue.

a. $\delta$ Hannover. G. v. Dr. Rüst.

b., c. $2 \sigma^{7}$ ad. Hannover. G. v. C. Braunstein.

d. alb. Hannover. G. v. Hofgrtn. Heiser.

e. f jur. Hannover. G. v. I)r. Mühlempfordt.

1020 (2832). T. senegalensis (L.).

a. Afrika.

1021 (2835). T. bitorquatus (Temm.).

Java.

1022 (2842). (halcophaps indica (L.).

a. ó borneo.

b. P Borneo. k. F.

1023 (2844). Pliaps elegans (Trmm.).

a. Australien. G. v. Konsul Kauffmann.

$102+$ (2847). Phlogoenas ('puenta (Lath.) Doldjitidetnube.

a. I'hilippinen. (i. v. Zooleng. Gartent. 
1025 (2848). Goura coronata (L.).

a. O' Nen-Guinea.

\section{Familie Carpophagidae.}

1026 (2850). Carpophaga aenea (L.).

a. - d. 4 o Bormeo. K. E.

1027 (2851). C. paulina (T'emm.).

a. 8 Celebes.

1028 (2851 a). C. pinon, selby,

a. $\sigma^{7}$ Neu-Guinea.

$1029(2851 \mathrm{~b})$. C. forsteni, Wall.

a. Oे Celebes.

10:30 (2859). C. bicolor (Siop.).

a. Bormeo. K. E.

1031 (2859 a). C. grisea, Gray.

a. ơ Bornen.

1030 (2860). C puella (less.).

a. of Nen-Guinea.

10:33 (2866). Ptilinopus purpuratus ((im.).

a, b. Australien. (i. v. HI. Grönes.

1034 (2868). Pt. superbus (Trmm.).

a. Amboirit.

1035 (2868a). Pt. rivolii, Gray.

a. 0 Nen-Guinea.

b. \& NeutGuinea. K. F.

$10: 36$ (2868 b). Pt. viridlis, Gray.

a., b. O'ర Bormen. K. E.

c. \& Borneo. K. L.

1037 (2868 (r). Pt. xanthorihous, Saled.

a. Oे Simghir-Inseln.

1038 (286sd). Pt. pulchellus, Bomp.

a. of Arru-luselu.

1039 (2873). Plapitreron leucotis (T'emm.).

а. of Tudiun.

10-10 (2877). Sphenocepeus oxyurus (Temm.).

a. of Borlueo.

10+1 (2879a). Trepon abyssinica, Cray.

a. Afrika. G. v. (:. Braunstrin.

10+2 (2855). Tr. amboincusis (Mïll.).

a., h. ठర Java.

('. P Javi .

2 Bïlgre 
10.1:3 (2088 ). Osmotrepon velumans (1.).

i., 1. ज̈' Jilvit.

e. Y Java.

104t (28s8a). Calocmas nicobalrica, Giay.

a. of Nen-Guimail.

\section{Familie Didunculidae.}

1045 (28s9). Didnu(nlus strigirostris (dard.).

a. Simnoit-Inschlı.

\section{Ordo Rasores, Sidarruöạl.}

Familie Gallidae. Häiflutux.

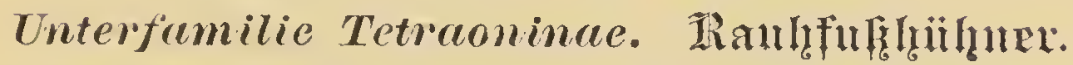

1046 (2890). Lagopus lagopus (L.) Mivoridurefingu.

a., b., c. Schiwriz.

1047 (2895). Tympanuchus cupido (L.).

a. o Nordamerika.

b. o Nordamerika.

1048 (2897). Bonasa bouasia (L.) 5̧a f(ffulfu.

a. of Europa. G. v. H. Voigt.

b. \& Europa. G. v. Oberstltnt. v. Wrede.

1049 (2898). Tetrao tetrix, L. Birfyuf)n.

a. ${ }^{7}$ Hannover. G. v. Dr. Voigt.

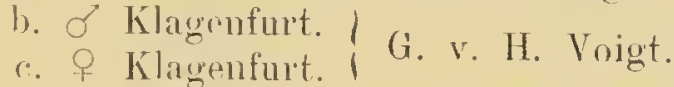

d. O IIannover'. G. v. ILofjüger Lewecke.

e. juv. Hannovere. G. v. Zoolog. Gartent.

1050) (2899). T. tetrix $\times$ T. urogallus. Ginfelfahu.

a. C Norwegerll.

$1051(2900) . \quad$ T. urogallus, L. 2tuerbalun.

a. of Niünden. G. v. Hauptm. Inechsler.

b. o Cassel.

r. O Hannover. G. v. Oberstltut. v. Islar.

d. pull. Hamover. (i. v. Oherwildnstr. Wallmann.

e. F Hamover. G. v. Major v. Lslar.

1052 2900 a). T. scoticus, Lith.

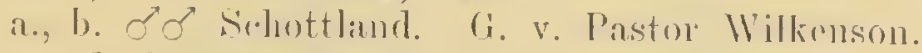

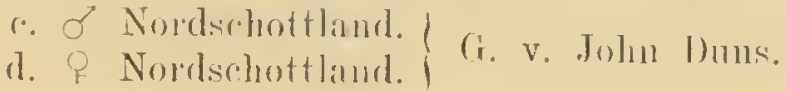


$1053(2900 \mathrm{~b})$. T. uralensis v. Middendorff.

a. $\sigma$ Sibirien. G. v. Dr. Rüst.

\section{Unterfamilie Odontophorinae.}

$105+(2903)$. Ortyx virginiamus (L.).

a., b. of Nordamerika. G. r. Zoolog. Garten.

c.-e. 3 \& Nordamerika. G. v. Zoolog. Garten.

$\rightarrow$ Bally.

1055 (2903 a). O. somninii, Steph.

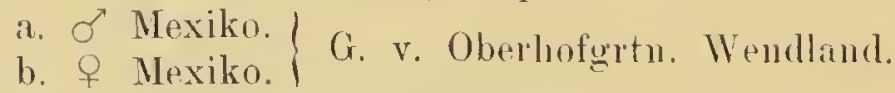

1056 (2907). Callipepla californica (Shaw).

a. o Californien.
b., c., d. 3 of Californien. G. v. Zoolog. Ciarten.

\section{Unterfamilie Perdicinae.}

1057 (2908). Rollulus poulpoul (sillops.).

a, b. ơ Java. G. v. Zoolog. Garten.

1058 (2910). Coturnix coturnix (L.) 2 sadftel.

a. Of Hannover. G. Y. G. Schwematum.

b. of Hannover.

1059 (2921). Caccabis saxatilis (Neyer). Stciulfulyu.

a. O Schweiz.

b. I sichweiz.

1060 (2922). C. saxatilis chukar (Gray).

a. $\sigma$ Cyper'il.

b. \& Cyperm.

1061 (2923). C. petrosa (Gm.) S(ipueulut).

a. of Corsicil.

b. + Corsica.

$1062(2924)$. C. rufa (L.).

a. $\sigma$ sizdfrankreicli.

b. \& Südffrankreich.

1063 (2925). Perdix perdix (L.) Micbluffu.

a. O Hannover. G. v. liraunstein jum.

b. $\sigma$ ad. Hannover. (i. v. Mdlrt. Hahm.

$\because$ of Hannover. G. v. C. Bramintein.

d. ot Ilannover.

e. juv. Hammover. \{ (x. v. Mdlrt. Hahn.

g. I Iamover. (i. v. Braunstein jun. 
lotit (29)2a) P. barbata, Verr.

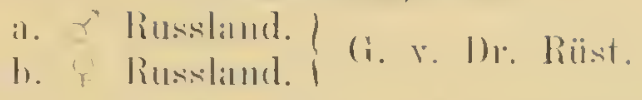

1065 (29:37). Francolinus frameolinus (1.).

a. of süileuropia.

b. \& siïleuropara.

Unterfamilie Phasianinae. Falancu.

1066i (2953). Numida vulturiua (landw.).

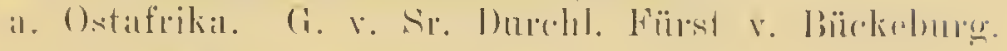

1067 (2956). Gallus varius, shaw,

a. Co Java.

b. \& Java.

1068 (2960). Euplocomus melauotus, Blyth.

a. Cl Iimilitya.

b. pull. Llmalaya.

1069 (2963). Eu. nycthemerus (L.) Silforfajau.

a. O' China.

1070 (2968). Phasianus colchicus, L. Gícucincr Fanjan.

a. ơ Ilannover.

b. \& Hamnover. (i. v. Dr. Rüst.

1071 (2971). Ph. soemmeringi, T+mm.

a. T Japan.

107일. (2972). Ph. wallichi, Hardw.

a. Of Nordindien. \{ (i. v. Pastor Wilkinson.

1073 (2973). Thamblea picta (1.) Boldfajan.

a. of China. (i. v. Fian Egestorff.

b. pull. China.

↔. $\Varangle$ China. (d. v. C. Braunstein.

Unterfamilie Pruminae. Pfanwu.

$107+(2978)$ Pavo cristatus, L. Bsmciucr Hifan.

i. In Inlipll.

b. Indien.

1075 (2979). Argusianus argus (h.) 9rguspfon.

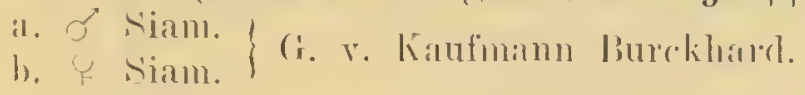

Unterfamilie Moleagrinac.

1076 (2982). Tragepan satyrus (1..).

a. Fepil. G. v. Waston Wilkinson. 
1077 (2983). Tr. melanocephalus (Gray).

a. F Himalaya. G. r. Zoolog. Garten.

1078 (2984). Lophophorus impeyanus (Lath.) (5ilnůajan.

a. of Himalaya.

b. of Himalaya.

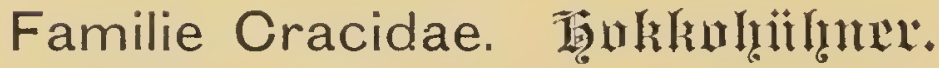

1079) (2996). Penclope superciliaris, Wagl.

a. Brasilien.

$1080(2997)$. P. pipile, (im.

a. Brasilien.

1081 (2997 a). P. cristata, Latl.

a. Westindien.

$1082(3000)$. Optalida motmot (L.).

a. Brasilien.

108:3 (3001). 0. aracuan (spix.).

a. $\sigma$ Brasilien
b., c. ot.q Brasilien.
1 Balg. v. Mr. Tölsner.

\section{Familie Megapodidae.}

1084 (3004). Megapodius forsteni, Trmm. ot Schlew.

a. Austratien. (i. v. II. Gröner.

\section{Ordo Gressores, Sdareituögel.}

\section{Familie Ardeidae. Avilgex.}

1085 (3007). Helodias alba galatea (Molina).

a. Ameriki. (i. v. Hauptman Niemeyer.

h. Australien. (i. v. Konsul Kauffmamm.

$1086(3008)$. H. prallei (Bj\%).

a. Mexiku.

$1087(3010)$. H. garzetta (1..) Ecibcurcilyer.

a.-d. Südusteuropa.

1088 (301:3). H. gularis (Bosco).

a. Sunda-luseln. K. li.

10s:9 (30)16). Ardea purpurea, L. Piurpurreifer.

a. O' Schweiz.

b. P Findren. 
$1090(3018)$. A. cincerea, I. Jijderciljer.

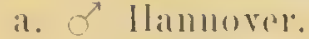
ь. $\alpha$ jux. Hamnover.
(a) O Hammover.
d. of Hammover. I G. r. Hh. Kohlialusch.
i. Hamnover. I
f. Hamover. (i. v. r. Limburg.

1091 (3018:1). A. lineata, l'1\%. Wiorl.
a. of Brasilion.
b. of Brasilien. k. k.
c. juv. Bratsilien. K. L.

1092 (3024). A. caerulea, L.
a. ad. Amerikil. K. E.
b. jur. Amerika. K. E.

$1093(3024 a) . \quad$ A. Scapularis, I11.

a., b. siidamerika.

$109+(302+b)$. A. erythromelas, Vieill.

a., b. Brasilien. G. v. Hlïttenmeistor Nolte.

1 Balg.

$1095(3024 \mathrm{e})$. A. candidissima, Gm.

a. Costarica.

1096 (3030). Ardeola speciosa (Horsf.).

a., b., c. Java.

2 Bülgre.

1097 (30131). Butorides vireseens (L.).

a.-1. Südamerika.

2 Bïlex.

1098 (3033). Ardetta minuta (L.) 3ucrgroljroumucl.

a. of Hilmurever. (i. v. Sasse.

b. Q Hammover. (i. v. Hiramles.

$1099(3034)$. А. cimmamomea (Gm.).

a., b. Java.

1100 (30:36). Ardeiralla flavicollis (Lath.).

a.. L. liomen. K. E.

1101 (3038). Botaurus stellaris (L.) Groigc Viobrommel.

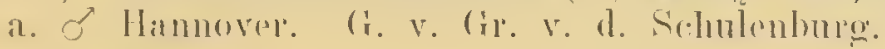

b. Hammovert.

r. t Llamover. G. v. Consist.-Rat IImupel.

d. fl Hannover. G. v. (ir. v. Bunigsent.

a. of llannover. (i. v. ( Wodekinrl.

1102 (3039). B. lentignosus (Mnntagn).

a. Amerikat (G. v. Konsul Nanue. 
$1103(3040)$. B. poeciloptilus (Wagl.).

a. Australien. G. v. Konsul hauffmann. 3 Biilge.

$110+$ (3043). Nycticolax nycticorax (L.) Madjtreiger. a. Europa.

1105 (3044). N. nycticolax naevins (Bodd.).

a. Amrika. (i. r. Ilüttemmeister Nolte.

$1106(3045)$. N. caledonicus (Gm.).

a., b. Australien. (i. v. Gröner.

(•) Australien. (i. v. Konsul Kauffmann.

1107 (3047). N. violaceus (L.).

a. Sïdamerikil.

b. juv. Südanmerika.

$1108(3050)$. Cancroma cochlearia (L.) Safujduaber.

a. Mittelamerika. K. L.

b., r. 2 jur. Nittelamerika. K. E.

\section{Familie Phoenicopteridae.}

1109 (3052). Phoenicoptelus posens, Pall. (Bicuciucr Jinniltgo.

a. Sardinion. (i. v. H. Fabian.

1110 (3052a). Plı. ignipalliatus, Geoffr.

i. $\sigma$ südeuropa.

b. \& süderuopla. (i. v. Zoolog. Garten.

1111 (30)52 b). Ph. ruber, I.

a. of ad. Chiti. ( G. r. Iliittrnmeister Nolte.

\section{Familie Ciconidae. Stirtultre.}

1112 (3055). Ciconia alba, l. Weciper Stord).

a. Y Hammover. G. Y. (Y. Braunstein.

1113 (3056). ('. nig̨ra (I.) Sdjwnrzer Sturd).

a. Of Ilamover. (a. v. Zoolog. Garton.

$1114(3057)$. C. abdimii, hirht.

a. O Nirika.

1115 (3067a). Tantalus mexicanus, Virill.

a. Nmerikil. (i. v. Hlïtrumeister Nolte.

$1116(3078)$. T. lactens, Raffl.

a. Jiva. 
1117 (3)669). T. Iocenlator, I.

a. Brasilicue (i. v. Lint. Whas.

\section{Familie Ibidae. Itrille.}

1118 (30-1). Platalea lencorodia, L. Luffetreiber.

a. limopra.

$1119(3073)$ P. aуjaja, l.

i. of Siidlatmeriliat.

b. sürlamerikil.

1120 (3078). Ibis candata (Bodl.).

i. ơ jux. Mittelamerikia. (i. v. Hüttemmeister Nolte.

b. T Chili. I G. v. Fabian.

r. $\rightarrow$ ('hili.)

1121 (3084). I. spinicollis, dimes.

a. dustratien. (G. v. Konsul Katuffmann.

1122 (3) 866$)$ I. romata, Eilı.

a. Afliki.

1123 (3089). Plegarlis rubra (L.) Moter Juis.

a. jur. Brasilien. K. E.

h. Brasilien.

$112+$ (3091). Pl. falcimellus (L.) Branucr Gidfler.

a. Europa.

\section{Ordo Crypturi.}

1125 (30192). Tinamus major (Gm.).

a., b. Brasilien.

1126 (3100). Nothoprocta perdicaria (kittl.). n. ('hili.

1127 (31013). Nothoura ma(culosa, Bomp.

a. Siidamerika.

\section{Ordo Alectorides,}

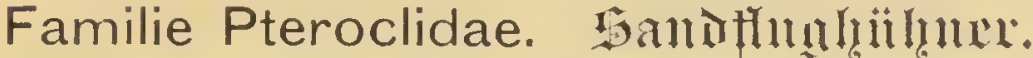

1128 (3102). Syrrhaptes paradoxus (l'all.) Stepuculuhu.

a. Amrum. (i. v. II. sielmulze.

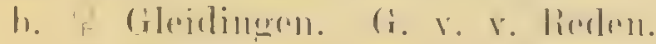

r. F Mïnder. (i. r. Forstdir. Burrkharel. 
1129) (3102). Pterocles arenarius (Pall.).

a. Y. Sinanicu.

1130 (3105). Pt. gutturalis.

a. Afrikit.

1131 (3106). Pt. alchata (L.).

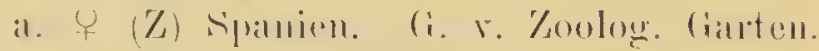

11:32 (3111). Pt. senegalus, L.

a. Ifrika.

b. Y Afrikia.

1133 (3112). Pt. colonatus (Licht.).

i. $\delta$ Afrika.

\section{Familie Otididae. Traxum.}

$1134(3120)$. Otis taloda, L. Gruje Trapue.

a. of Hannover. (i. v. (ir. v. Bennigresen.

b. of Hannover.

(c. \& Hamnover.

1135 (3129). O. tetrax, L. 3ucrgtrappc.

i. O Eiropia.

b. + Furopa.

Familie Gruidae. Hamitngt.

1136 (3136). Baleariea pavonina (L.).

i. of Westafrika.

1137 (3137). Grus grus (L.) Sranid).

a. Of Itamover. (i. v. Gr. v. d. Selhulenhury.

b., c. 2 pmll. Hammoser.

d. + Hannover. G. v. Fierleler.

r. of llammorer.

$113813141)$. G. calluculata, Gim.

a. Siirliafrika. (i. r. Rocholl.

1139 (3141 a). G. moxicana, Briss.

a. jur. Mrexilo. (i. r. Zoologe. Giatent.

$1140(31+3)$. (ir. virego (L.).

i. Of Dobrurkeclai.

Familie Eurypygidae.

1141 (3144). Eurylyga solaris (Bode.).

a. Surintm. K. L. 


\section{Familie Rallidae. Lallen.}

1142(31+6). Ilelioruis fulica (Bodll.).

a. Surinam. k. li.

$11+3(3147)$. Fulica ristata, (im.

a. Sïrlatirilin.

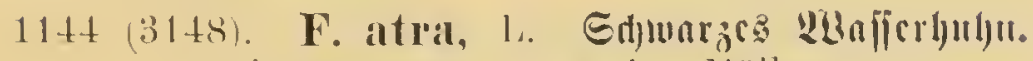

a. of Hammover. (i. v. Mr. Miïller.

b. of llammorer.

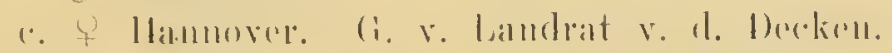

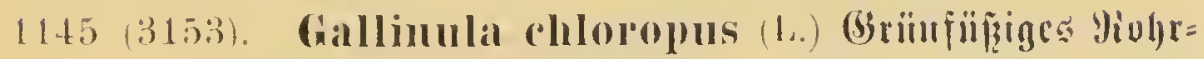
()III)II.

a. of Hamnover.

b. of Hannover. (i. v. Arigerstein.

๕. jur. Hannover. (i. r. C. Braunstein.

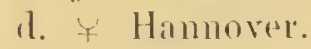

$1146(3153$ a). G. chlopopus $\times$ F. atra (L.).

a. Hannover. (i. v. Kreye.

$11+7(3153 \mathrm{~b})$. G. galeata, Prz. Wied.

a.. b. Brasilien.

$11+8(3153 \cdot)$. G. vus, Giriy.

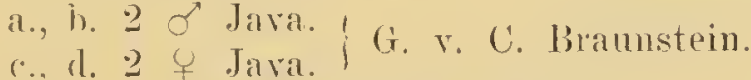

$1149(3155)$. G. phoenicura (I'enu.)
a. J Java.
h. Tova.
3 Bäluge.

1150 (3155a). G. flavipostris, Bonp.

a., h. Surinam. K. F.

r. pull. Surinam. K. E.

1151 (3156). Porphyrio veterum, (im. \&urpurbul)u.

a. Südeuropa. G. v. Götting. Nuseum.

b. Sürleuropa.

1152 (3158). P. melanotus, Temm.

i., h. Bornew. K. li.

1153 (3161). P. martinieus (L.).

a. Surinam. K. k.

h. Brasilien (i. v. Majol v. Tslar.

$115+(3163)$. Ontygonetra capolina (l..).

a. $\int$ Norrlimmerika.

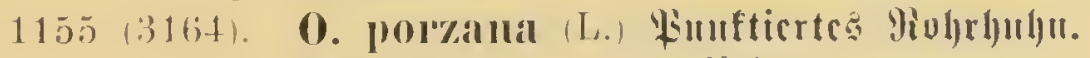

a. of Hannovere. (i. v. Mr. Voight.

b., e. llannover. 


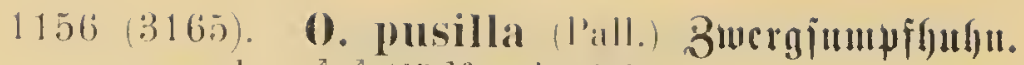

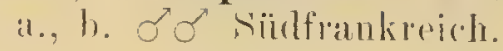

1157 (3177a). Rallina erythothorax, sidhleg.

a. Sunda-Inseln.

1158 (:3179). Crex clex (L.) Miandelfüng̣.

a., h. उo sochlesien.

a. d Ilammover. (i. v. Hofjiginer liuse.

d.-f. 3 pull. Ilannover.

«.. Y Hannover. (i. v. Dr. Nühlenpfordt.

2 Biilge.

1159 (3186). Rallus longipostris, Bodrl.

a. Surinam. K. li.

h. Surinam.

1160 (3189). R. niendeans, vieill.

a. Sürdamerikal G. v. Hüttrometr. Nolte.

b. Ұ südanerika. (i. r. Dr. T'ölsner.

1 Balg.

1161 (3191). R. aquaticus, L. Biajicrufle.

a. Of Hamnover. (i. v. Biramles.

b. of Hammover.

(. + Hamnovel.

$1162(3194)$. R. virginianus, L.

a. Nordamerika.

1163 (3195). Almides cayennensis (Gm.).

i. S Surinam. K. li.

b., r. 2 pull. Surinam. K. F.

d. Y surinam.

\section{Ordo Limicolae.}

\section{Familie Parridae.}

116 (3) (3202). Parral indiea, Latl.

a. killkutta.

$11(65)(3205)$. P. jacalla, H.

a., 1. 2 of surinam. k. li.

(a) do Brasilien.

1. of juv. Surinam. K. li.

e. Sullintm K. li.

2 Biilge (1 G). 


\section{Familie Turnicidae.}

$116(6)(32017)$. Tumix sylvatical, Dosfout.

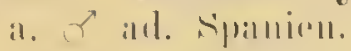

1167 (32010). T. pugnax ('T'emmo).

it. 7 c'cylont.

\section{Familie Thinocoridae.}

11 (is (3214). Thinocorus pumicivopus, lischisch.
a. of (hili.
b. Chili. I (i. r. Fabian.

1169 (3214a). Attagis gayi, Geoffr.

a. of Chili. I G. v. Fabian.

\section{Familie Charadriidae.}

1170 (3215). Vanellus vanellus (L.).

a. o Hannover. (i. v. Ohergrrt. Dommes.

b. pull. Hannover. G. v. Behnke.

c. \& Hanmover.

1171 (3215a). V. cinctus, Schley.

a. Australien. Gr. v. Dr. Müller.

1172 (3217). V. cayenuensis (Gm.).

a. Südamerika, G. v. Hüttenmstr. Nolte.

h. Südamerika.

1173 (3219). Chettusia gregaria (Pall.).

a. Tolea.

1174 (3231). Lobivanellus lobatus (Lath.).

a. Australien.

1175 (3235). Chapalrius pluvialis, L. Guloregeupfeifer.

a. of Hannover.

b., c. 우을 Hannover.

$1176(3236)$. ('h. dominicus fulvus (Gm.).
a., h. Borneo. K. E.
(c. Borneo.
l., e. Girönland (०).
1 balu.

1177 (32365). (H. marmoratus, Watrl.

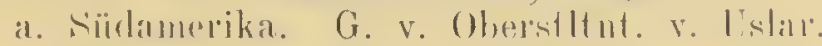

b. Sürlamerika. G. v. Obergrtt. Dommes. 
1178 (32:37). Ch. squatarola, L. (varius). Sicbizzegen= pfifer.

a, b. Spiekerong.

1179 (3237a). Ch. ruficollis, Wagl.

a., b. Südamerika. (i. v. Fabian.

$1180(3240)$. Ch. morinellus, I. Miorneflegenpfeifer.

a. of ad. Schwedent.

b. $q$ ad. Stchweden.

1181 (3241). Ch. vociferts, L.

a. Nordameriki. G. v. Ltnt. Wehr's.

$1182(3243)$. Ch. biciuctus, Jard. et Selby.

a. Australien.

1 Balg.

$1183(3243$ a). Ch. monachus, (ieoffr.

a. Australien. (x. v. I)r, Müller.

1184 (3245). Ch. alexandrinus (L.) Eecregenpfifer.

a. $\sigma$ sipiekeroog.

b. o spiekeroog.

1185 (3250). Ch. hiaticula, l. Eaubregeupfeifer.

a. O Hannover. (i. v. C. Braunstein.

b. ơ Amrum.

r., d. 2 juv. Amrum, (i. v. I)r. Rüst.

1186 (3254). Arenaria interpres, L. Etcimuärger.

a. O" ad. Schweden.

b. \& arl. Sishwerden.

1187 (3255). Haematopus ostrilegus (L.) Aujterufijdjer.

a. ơ spickerong.

h., c. 우 sirickeroog.

1188 (3259). Cursorius galliens (Gm.) $\mathfrak{B a j p t e n l u ̈ u f e r . ~}$

a. Sürleuropra. (․ v. v. Bodemeyer.

1189 (3066). Glareola pratincola (L.) Bradjidunnlbe.

a., b. Griechenland.

Familie Oedicnemidae. Difffiilygt.

$1190(3270)$ Oedienemus cerppitans (L.) Tricl.

a. Of Eimopa. (i. v. Silsse.

b. ㅇ Europa. 


\section{Familie Scolopacidae.}

1191 (327s). Recurvirostral alvosetta, 1. Gäbcljann̈brer.

i. of binten. (i. v. ('. Bramustein.

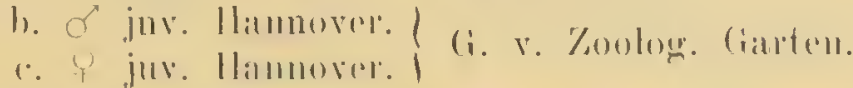

1192 (3282). Ilimantopus blasiliensis, Brehm.

a. Nittolamerikis. (i. v. L, tut. Wehrs.

1193 (328:a). II. leurocephalus, (ionld.

a. Australien. (i. r. D)r. Miiller.

$119+$ (3284a). Phalaropus hyperboreus, lath.

a. Hoher Nordin. (i. r. C. Braunstein.

1195 (3285). Calidris arenaria (L.) Emocrling.

a. or Surekerooy. (i. v. D)r. Armbrust.

b. of spiekeroog.

1196 (3288). Limicola platyrhyncha (Temm.) Emmpi= länกัcr.

a. Amrum. (G. v. Dr. Rüst.

1197 (3289). Tringa temmincki, Leisl.

a. O' ad. Sithwerden.

b. F ar. sichweden.

1198 (3290). Tr. minuta, Leisl. Bucrgjtrandläufer.

a. Nordsee-Inseln.

1199 (3293). Tr. alpiua, L. Mupcuitrandünfer.

a., b. उo spiekeroog.

c.. d. $\sigma^{\top} \sigma^{\prime}$ Hannover.

e. $\delta$ Nordamerika.

f. F Sipiekeroog.

$1200(3294)$. Tr. subareuata (Gïld.) Bogenjdun̈briger Strandrïnjer.

a. $\sigma$ ad. Sïdfrankreich.

b. \& arl. Südfrankieich.

1201 (3295). Tr. maritima, Brïnn. Eccjtranbläufer.

a. ¿ spiekerongr.

b. Of Hannorer. (i. r. Fr. r. d. Deckent.

1202 (3296). Tr. australis, Jard. of Selhy.

a. Anstralien.

1203 (3296;). Tr. rufescens, Vicill.

a. Sïramerika. (t. v. Ohrergert. 1)ommes.

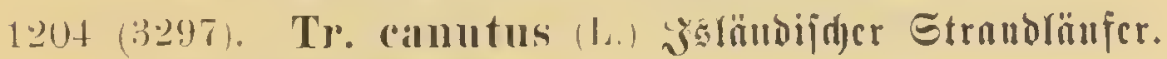

a. 3 ad. sidhweden.

b. + ad. sichweden. 


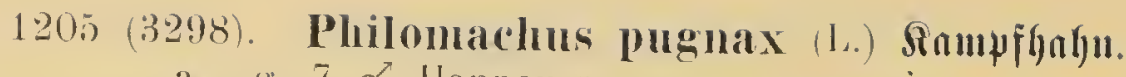
a.- g. 7 o Hannover.
h., i. 2 \& Hannover.

1206 (3299). Totauns hypoleucus (L.) Flup̧uferläujer.

a. of Hannover. ( i. r. C. Braunstein.

c. jur. Hannover. (x. v. Zoolog. Garten.

$1207(3300)$. T. glareola (L.) Brudjwajjerlüufer.

a. of Hannover. (i. v. Dr. Hahn.

b. of Hannover.

c. + Hannover. G. v. Rer. Benecke.

1208 (3301). T. ochropus (L.) 23 alomajïerläufer.

ஃ. ơ ad. Ungarn.

b. T⿱ ad. Ungarn.

(C. mit Nest, Hannover. G. v. H. Stolberer.

1209 (3302). T. calidris (L.) Jiotjdenfel.

a. O' Spirkeroog.

b. of Hannover. (G. v. Ditzen.

1210 (330:3). T. staguatilis, Bechst. Teidjunjīerläufer. a., b. Ungarn.

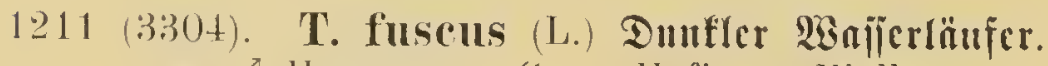

a. O' Hannover. (i. r. Ilofjïger Wallmann.

b. of Hannover.

¿ o Hannover. (i. v. C. liraunstein.

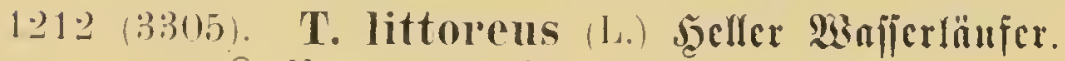

a. O Hannover. (i. v. Ltut. v. Uslar.

1213 (3306). T. melanolencus (im.).

a. Amerika. (i. v. Fabian.

$1214(333) 6$ a $)$. T. glottis, 1.

a. if llannover. (G. v. litnt. v. Uslar.

121:5 (3:308). T. macularius (1..).

a. Nordamerikir.

1216 (3:310). Heteractitis incaums (Gmo).

i. of Bornoo. K. L.

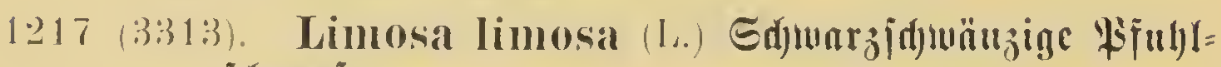
id)uepfe.

a. ఫ’ ad. Hannorer. li. v. (i. v. Düring.

b. juv. Borkum. (i. r. Biraunstein jun.

(‘. Z ad. Ilannover. 1). r. Ditzen.

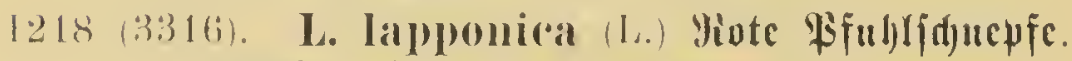

it. Nitale. (i. v. C. Braunstein. 


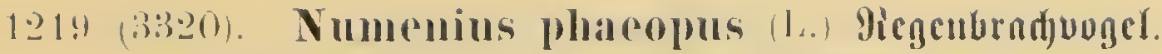

a. Melgoland (i. v. ()herförster Ilesser.

b. (Istindion.

(.) Bormen, K. F.

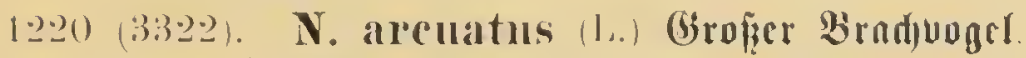

a. o Hatmunoril.

b. of Hammovers. (i. v. liber's.

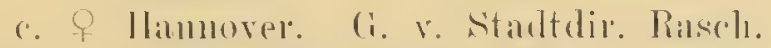

12.21 (3:32:3). N. Iongirostris, Wils.

a. Amrikil. K. E.

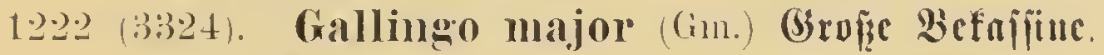

a. of Himmover.

b. o Hammover. G. r. Dr. Mühlempfordt.

c. of Hamover. (G. v. Stolberg.

1223 (3325). Gallinago gallinago (L.) Befajifuc.

a. Of Ilammover. \& G. v. Pralle.

c. pull. (i. v. Dr. Hahn.

d., e. Hannover. G. v. v. Hedemamn.

$122+(3325$ a). G. brehmii, Kaup.

a. Göttingen. G. v. v. Hedemann.

1225 (3331). G. frouata (HII.).

a. Brasilien. Gr. v. Dr. Tülsner.

b. Surinam.

c. Siidamerika.

2 Bälge.

1226 (3336). G. galliumla (L.) Sleme Befajifite.

a., b., c. Hannover.

d. Of Dollbergen, Hannover. (i. v. Carl Rüst.

e. ol Hamnorer. G. v. C. Pramnstrin.

1227 (3337). Scolopax rusticula, L. $\mathfrak{3 a r d o j d u c p f e . ~}$

a. Ol Iannorer. G. v. Forstrat Mühry.

b. \& Haunover. G. v. C. Braunstein.

1228 (3342). Rhynchaca semicollaris (Vicill.).

a. Niidamariks. G. v. Ilüttemmstr. Nolte.

1229) (3342 a). Rh. australis, (ioull.

a. Austrilien. (i. v. M'rofessor Miillere.

Ordo Lamellirostres.

Familie Palamedeidae.

$12: 30$ (3:3.3). Palamedea rolnuta (1..).

a. Of Inasilinn. (r. v. I)r. Tülsmer. 


\section{Familie Anatidae.}

1231 (3345). Cygnus olop (Gm.) jeüberjufuma.

a. Llammover.

b., c. 2 pull. Hannover'.

1232 (3346). C. cygnus (L.) Singidjunu.

a. Hamnover.

1233 (3347). C. bewicki, Yarr. Bwergiduman.

a. Südamerika.

$12: 34$ (33:348). C. atratus, Lath. Ed)warzer Edjwnu.

a. $\sigma^{\prime}$ Australien.

b. pull. Australien.

c. Of Australien. G. v. Zoolog. Garten.

d. P Australien.

12:35 (3351). Cereopsis novaehollandiae, Lath.

a. $\sigma^{7}$ Austratien. G. r. Zoolog. Ciarten.

12:36 (33566). Anser anser (L.) (3raugans.

a. $\sigma$ Wolga.

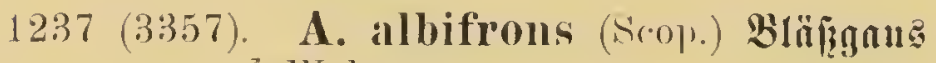

a. Oo Wolgan.

1238 (3358). A. segetum (Gm.) Enatgnus.

a. of Hannover. G. v. Dr. Hahn.

b. o juv. Hammover.

r. of llammover.

d., e. of o Hannover.

1239 (3361). Branta canarlensis (L.).

a. of Nordameriki.

1240) (33362). B. melanoptera, byton.

a. Chili. (i. v. Fabian.

1241 (3336(i). B. bernicla (1.) Mingelgam.

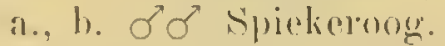

c. jux. Ritzebütel. (i. v. Major Gade.

$12+2$ (3367). B. lencopsis (Bentert.).

i. of Thutsedliand. (i. r. Zoolog. Garten.

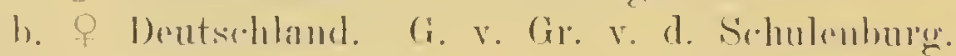

12.1:3 (33370). B. jubata (Lath.).

a. Anstralien. G. v. Professor Mlüller.

1244 (3:370a). Anseranas melanobenca, Lath.

a., b. Austrilien. (i. v. (iröner.

1245) (3332). ('hemalopex acoyptiacus (1.).

a. Afrikit. 
1246 (33375). Nettapus albipemmis (comll.).
a. ₹r jur. Bormeo.
b. of Anstralien.
r. Australien. Fo r. Professor Miillar.

1247 (3:376). Tadorna tadorna (L.) Brandgane.
a. O' I'slatr. Hannover.
b. O' Spiekeroog.
'., d. 2 pull. Hannover. ! G. r. Zoolug. Garten.

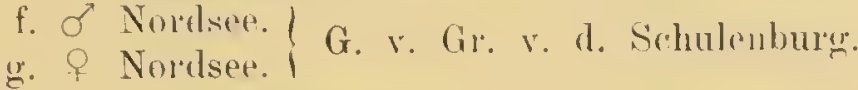
h., i. 우우 Spiekeroom.

1248 (3377). T. casarca (L.) Rojtgnı
a. of Russliud.
b. ㅇ Russland. ।
(. \& Russland. G. v. Zoolog. Garten.

1249 (3381). T. radja (Garuot.).

a. of Australien.

1250 (3386). Dendrocygna fulva (Gm.).

a., b. O' $\sigma^{\prime}$ Mittelamerika.

¿. Nittelamerika. K. E.

d. † Vittelamerika.

1251 (3390). Aix sponsa (h.) Brautente.

a., b. q o Nordamerika.

1252 (3391). A. galericulata (L.) Mambariucutc.

a. Of Ostindien. G. r. Zonlog. Garten.

1253 (3392). (airina moschata (L.) Miojduscutc.

a. of Australien.

1254 (3394). Anas boschas, I. Stoffente.
a. O Australien. G. v. Gröner.
b. of Göttingen.

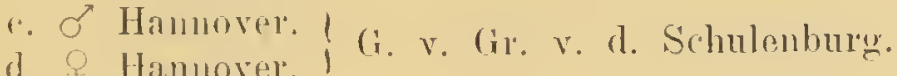
e. \& Hanuover.

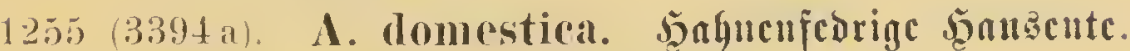

a. Hannover. (i. v. H. Grabow.

1256 (3399). A. specularis, King.

a. O' Chili. G. v. Fahiam.

b. of Chili. ( (i. v. Hlïttenmstr. Nolte.

c. \& ('hili. I

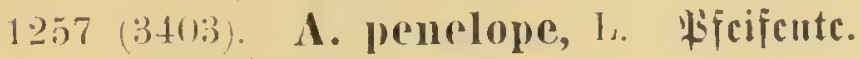
a. O’ ad. Hamnover.
b., ‘. 2 ơ juv. Hamover. 
$\left.\begin{array}{l}\text { d., e. } \sigma \sigma \text { Hamnover. } \\ \text { f. }+ \text { t Hamover. }\end{array}\right\}$ (i. v. Gr. v. d. S.hulonburg.

$1258(3406)$. A. angustirostris, Ménétr.

a. of Südspanien.

b. of Südspanien.

$1259(3407)$. A. chiloensis, King.

a. \& Chili.

1260 (3408). A. strepera, L. Edjuttercute.

a. o Hannover.
b. of Hamnover. G. v. Gir. v. d. Schulenburg.

c. f EIze a. d. L. G. v. Engehansen.

1261 (3410). A. crecea, L. Sirifente.
a. $\sigma$ Hamnover.
b. \& Hannover. G. v. Gr. v. d. Schulenhurg.
c. 8 Hannover.
d. \& Hamnover. (i. v. v. Hedemann.

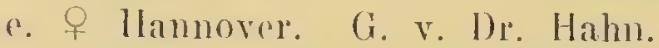

1262 (3+11). A. querquedula (L.) Sü̈feute.
a. of Itamorer.
b. \& Ilannover.
c. of Hannover, (i. v. Gi. v. d. Sechulenbure.
d. O Hannover. G. v. v. Hedemamm.

1263 (3+12). A. discors, L.

a. $\sigma$ ad. Nordamerika.

b. $\sigma$ jur. Mexiko. G. Oberliofyertn. Wendlant.

(r. T Mexiko.

1 Bale.

$126+(3+12$ a). A. falcata, Pall.

i. $\sigma^{7}$ Asimn.

b. A Asien.

$1265(3+12 b)$. A. formosa, Grorgi.

i. I Amur.

$1266(3+12 \%)$ Querquedula glocitans, kay.

a. Sürlammerika.

1267 (3420). Dafila hahamensis (L.).

a., b. бo lirasilien.

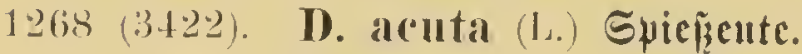

a. of Hannover. (i. v. Lecibjiager Palpe.

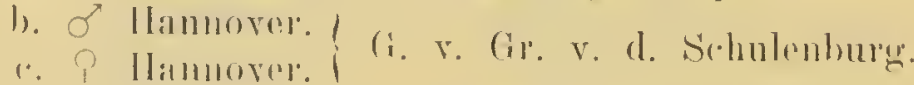

d. \& Dollbergen, Hamover. G. v. (arl kï̈st. 


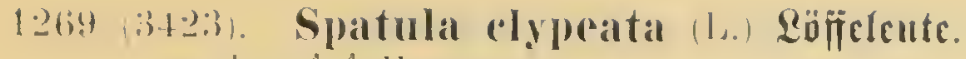

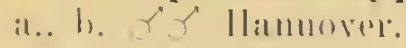

ध. o jur. Ilamnoser. (i. v. Gir. v. d. Sichutenhurg.

d. ₹ IImmover.

1270 (34.24.). S. rlyynehotis (Lath.).

a. of Australien. (a. \&. II. Gröner.

$1271(3425)$. S. platalea (Vieill.).

a. of Chili.) (a. v. Fabian.

$1272(3428)$. Fuligula fuligula, 1. Meiberente.

a. of IIannover.

b. of Derneburg. (i. v. Gr. Münster.

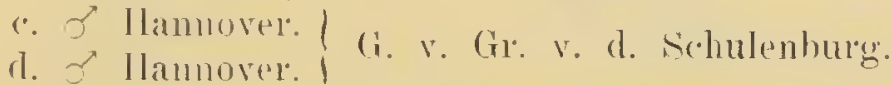

e. F Hannover. (i. v. Zoolog. Garten.

$1273(3429) . \quad$ F. marila nearetica, Stejn.

a. Of Hamover. G. v. Gr. v. d. Schulenburg.

b., c. 2 o juv. Lübeck.

1. क Lüberk.

e. F Hannover. G. v. Gr. v. d. Schulenburg.

1274 (3431). F. clangula (L.) Sd)cflcute.

a. $\sigma$ jur. Hannover. G. v. Dr. Rüst.

b. of Nienburg. G. v. H. liettler.

$\therefore$ \& jur. Hannover. (i. v. C. Brannstein.

d. Of Hamover.

f. Z Hammover. G. v. Obergirt. Dommes.

1275 (34:32). F. albeola (1..).

a. of England. G. v. Gir. v. d. Schulenburg.

$1276(3432 a)$ F. islandica, Gm.

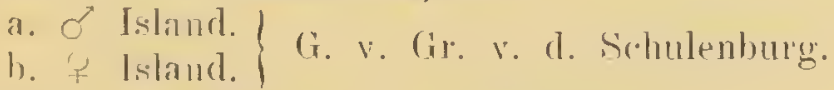

1276 (34:33). F. forina (1..). Tafelente.

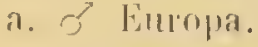
ค. of jur. Europa.
(.) F Europa.
i. f jur. linmepis.

1278 (3435). F. nyrocal (rï̈denst.) Mioorente.

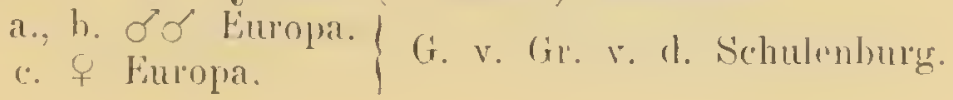


1279 (3438). F. rufina (Pall.) Solvenente.
a. O' Europa.
b. \& Europa.
G. v. Gr. v. d. Schulenburg.

1280 (3439). Clangula hyemalis (L.) (siżcute.
a. $\sigma$ Spiekeroog.
b. o juv. Spiekeroog.
c. O Lübeck.
d. ơ juv. Lüberk.
e. 우 Lübeck.
f. \& Hannover. G. v. Gr. v. d. Schulenhurg.

1281 (3440). Histrionieus histrionieus (L.) Sarlefinente. a., b. $\sigma^{7}$ Nordeuropa.
c. $q$ Nordeuropa.

1282 (3441). Eniconetta stelleri (Pall.) Enjeffente.

a. O' Nordeuropa. G. v. Gr. v. d. Schulenhurg.

1283 (3442). Somateria mollissima (L.) Eiberente.

a. O Hannover. G. v. Gr. v. d. Schulenburg.

b. ㅇ Döhren, Hannover. G. v. C. Braunstein.

1284 (3443). S. spectabilis (L.) Fradteiberente.
a. O' Nordeuropa.
b. juv. Nordeuropa. G. v. Gr. v. d. Schulenburg.
c. Q Nordeuropa.

1285 (3444). Oidemia nigra (L.) Traucrette.
a. $\sigma$ Lübeck.
b., e. $\sigma^{\top} \sigma^{\top}$ Nordsee. | G. v. Gr. v. d. Schulenburg.
d. \& Nordsee.
e. ㅇ Lübeck.

1286 (3445). O. fusca (L.) Sammetente.
a. $\sigma$ Insel Juist.
b. $\sigma^{7}$ juv. Grestemünde. (i. v. Dr. v. Bodemeyer.
c. o Kiel.
d. 9 Kiel.

1287 (3446). O. perspicillata (L.) Brillenente.

a. of Nordamerika.

1288 (3447). Biziura lobata (Shaw.).
a. of Australien. h. \& Australien. G. v. Marwede.

1289 (3447 a). B. armata.
a. O Südamerika.
b. of juv. Südamerika.
c. \& Südamerika. 


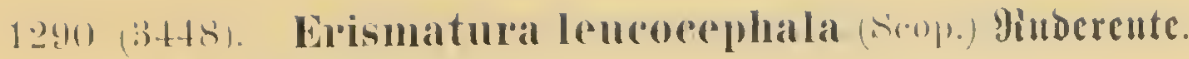

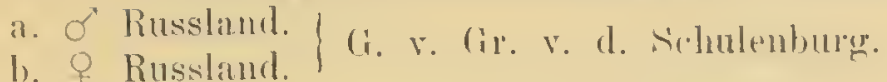

1.2!) 1 (3451). Melgus merganser, L. Gönjejüger.

a. of Hannover.

b. o' Haunover. G. v. (ien. v. d. Osten.

(a) juv. Hamover. (t. v. Oberegrert. Dommes.

d. \& Ilannover. Fr. v. Dr. Voigt.

e. F Hannover. G. v. Wallmann.

f. \& Hamnover. G. v. Fiedeler.

1292 (3452). M. selpatol, L. Mittlerer Gïger.

a. O Spiekeroog. G. v. Dr. Armbrust.

b. \& Hannover. G. v. Dr. Rüst.

c. O Hannover. G. v. Fiedeler.

1293 (3453). II. albellus, L. 3เwcrg|äger.

a. $\sigma$ Hannover. G. v. Gr. v. d. Schulenburg.

b. O Hamnover. G. v. Gr. Grote.

c. $\sigma^{7}$ juv. Eimbeck. G. v. Hauptun. Gade.

d. of juv. Hannover.
e. \& Hannover.

\section{Ordo Steganopodes.}

\section{Familie Pelecanidae.}

1294 (3456). Pelecanus l'ufescens, Gim.

a. Afrika.

1295 (3460). P. crispus, Bruch.

a. Afrika.

1296 (3462). P. erythrorliyuchus, Gin.

a. 우 Nordamerika. G. v. Ltut. Wehrs.

\section{Familie Fregatidae.}

1297 (3463). Fregata aquila (L.).

a. Tropische Neere. (i. v. Konsul Nanne.

h. Tropische Nere. G. v. Apotheker Stiunke.

\section{Familie Sulidae.}

1298 (3464). Sula bassana (1..) Bajztöluel.

a. Europa. G. v. Sr. Maj. König (ieorg. 
1299 (3467). Sula piscator (1.).

a. (ia) Horn. (i. v. C. Braunstein.

$1300(3+67$ a). S. fusca, Briss.

a., b. Amerika.

\section{Familie Phalacrocoracidae.}

\section{1 (3469). Phalacrocorax (Graculus) carbo (I..)} Siormurnu.

a. $\sigma^{7}$ Nordsee. (i. v. Amtsrat Lueder.

h. $\sigma$ Europa. (i. v. Zoolog. Garten.

( $\sigma$ Australien. G. v. Exc. v. Malorti.

d. F Hannover. G. v. Hanehuth.

a. O Nordamerika. (i. v. Götting. Nuseum.

1302 (3476). Ph. brasilianus (Gm.).

a. Brasilien. G. v. Oherort. Dommes.

1303 (3479). Ph. pygmaeus (Pall.).

a. Afrika.

1304 (3482). Ph. melanoleucus (Viell.).

a. Australien. (i. v. Mr. Müller.

1305 (3+82a). Ph. sulcirostris, coutd.

a., h. Anstralien. (j. v. H. Gröner.

1306 (3482h). Pli. lencogaster, (iray.

a. Australien.

1307 (3483). Anhinga novaehollandiae (Gonld).

a. Australien. G. v. Gröner.

$1308(3+85)$. Amhinga (L.).

a., b. Siidamnerika. (i. v. Konnsul Nanne.

( Nexiko. (i. v. Oberhofgrtn. Wendland.

Familie Phaëtontidae. Truytiküngel.

1309 (3486). Phäiton aethereus (T..) Tropifuogel.

a., h., r. Thropische Mrere.

Ordo Longipennes, \$erfliegrer.

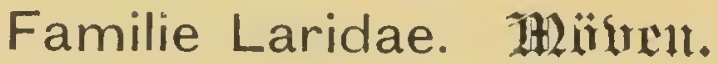

1310 (3494). Anous stolidus (1.).

a. Westkïste von Nordamerika. 
1331 (3497). Ilydrochelidon lencoptera (s.thin\%).

a. souidfrankiraich.

1312 (3.98). H. hybrida (Pall.).

a. of südfrankreich.

1313 (34499). II. nigla (L.) Edjunrze Secjdunaluc.

a. O' Hammover. (i. v. (¿. Riiist.

b. Himmover. (i. v. Ditzen.

1314 (3503). Sterna minuta, l. Bwergiecjumalbe.

a. Nordimerika. (i. v. (). Branustein.

\begin{tabular}{lll|l} 
b. of Amrum. & (i. v. Dr. Rüist. \\
(. of & Amrum.
\end{tabular}

1315 (3505). St hirundo, 1. Frupjecidumaltue.

a. o Hammovere.

b. Hamover. (G. v. Dr. Struckmann.

c. Hannover.

d. O Spiekerong.

e. \& jur. Hannover. (ix. v. Hauptm. v. Issas.

1316 (3507). St. macrura, Naum. Sinjtenjecjdunfluc.

a. of ad. Norwegen.

h. f ad. Norwegen.

1317 (3507 a). St. anglica, Montague.

i. $\sigma$ ad. Südfrankreich.

b. $q$ ad. Südfrankreich.

1318 (3516). St. media, Horst.

a. O' Marokko.

b. Marnkko.

1319 (3517). St. cantiaca, Gm. Brandecidjundbe.

a., b. Enropa.

1320 (3519). St. bergii, Licht.

a. f Indien.

1321 (3520). St. tschegrava (caspia. Pall.) Lepeseh.

a. $\sigma$ Fintand.

b., c. $\sigma^{7} \sigma^{7}$ Ammunn. G. r. Forstm. Hess.

d. Anrum. G. v. Obreförster llesste.

1322 (3522). Larus minutus, Pall. Bucrgüuc.

a. of Helgoland. G. v. H. Stollerer.

b. of Sarepta.

132:3 (3524). I. ridibundus, I. Indjüüe.

a. of Europar. (i. r. H. Bithe.

b. R Europa (t. v. Leilmerl. Baring.

1324 (3526). L. novaehollaudiae, Steph.

a., b., c. Australien. (i. v. Konsul Kanffimann. 
1325 (3529). L. Serranus, Tsch.

a. St Sürdamerika. G. v. Fabian.

1326 (3533). L. franklini, Sw. et Rich.

a. Mexilio. G. v. Ltut. Wehrs.

$1327(35: 35)$. L. gelastes, Licht.

a. $\sigma$ Syrien.

1328 (3536). L. camus, L. Sturmmöve.

a., b. бర Spiekeroog.

c. juv. Spiekeroog.

I. ㅇ Spiekeroog.

$1309(35: 39)$. L. fuscus, L. Scringånüve.

i. Steliweden.

$1: 330(3540)$. L. argentatus, Brün. Gilbernüve.
a. Norderney. G. v. Dr. Oehlrich.
b. juv. Spiekeroog.
r. $Q$ juv. Elbe.
d. 우 Spiekeroog. G. v. Obergrrt. Dommes.
e. S Spiekeroog.

$1: 331$ (3541). L. dominicanns, Licht.

a. Sürlamerika.

1332 (3543). L. marinus, L. Grofic Minntelmöuc.

a. O" Nordsee. G. v. Hofrat Berthold.

b. $q$ juv. spiekerong.

c. ㅇ Nordsee. G. v. Ltnt. v. Hinüber.

1333 (3543 a). L. belcheri, Vigors.

a. Nittelamerika. G. v. Hüttemnstr. Nolte.

$1: 34$ (3546). L. ichthyaeitus, Pall.

a. O Kaukasus.

13:35 (3547). Gavia alba (Gum.) Grff́cutbcimübe.

a. Of Grönland.

1336 (3548). Rissa tridactyla ( $\mathrm{I}_{\text {. }}$ ) Dreizeljeumäuc.

a. Oo Bantelı. (i. v. Gr. v. Bemmigsen.

b. o" spiekeroog.

(․, d. of of spiekerong.

13337 (3549). Chema sabinii (Nab).).

i. juv. Ciröuland.

1338 (35550). Stererarius parasiticus (1..) Sdfunrotzer= rantumïve.

a. of Hammover. (i. v. I'ralle.

b. of Nordsee. (i. v. Ditzon.

c. ㅇ Hamnover. G. v. () Braunstein.

d. Pammover. G. r. lir. Sicheele. 
1:339 1:3551\%. St. pomarinus (Temm.) Mittlere Mianbüue.

a. Har-Oer.

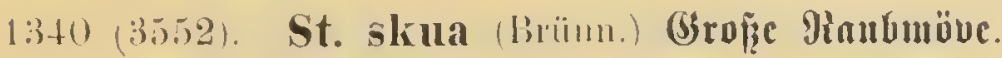

i. of Far-()er.

134+1 (3552a). St. cepluus, Blas.

a. S Norweyen.

\section{Familie Procellariidae.}

1342 (355t). Diomedea chlororhyncha, Gm.

a. Sïill. Ozean.

$1343^{\circ}(3555)$. D. culminata, Gould.

a. Nüdamerika.

$134+(3555$ a). D. cauta, Gould.

a. O Cap Horn. G. v. Dr. Rüst.

b. + Südamerika.

(•. Südl. Ozean. G. v. I)r. v. Bodemeyer.

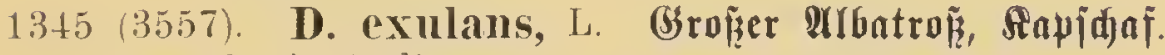

a., b. Australien.

( Cap Horn. G. v. Dr. Rüst.

1346 (35ว9). Fulmarus glacialis (L.).

a. ơ Far-Oer.

b. + Nordeuropa.

1347 (3560). Majaqueus aequinoctialis (L.).

a. Australien.

1348 (3561). Daption capensis (L.) Raptaube.

a. Südamerika.

b. Cap d. g. Hoffinung. G. v. Dr. v. Bodemeyer.

c. City d. g. Hoffnung. G. v. Dr. Mühlenpfordt.

1349 (3563). Puffinus major, Faber.

a. of Malaga.

b. (iriechenland. G. v. Dr. Krïper.

1350 () (3565). P. puf finus (Brünn).

a. ơ südtrankreich.

1351 (3565a). P. carneipes, Guuld.

a. Australien. (․ v. Dr. Müller.

1352 (3571). Procellaria pelagica, 1. Gómeine Sturm= idjualue.

a. $\sigma^{7}$ Europa. 


\section{Ordo Urinatores.}

\section{Familie Podicipidae. Theitgtitipe.}

1353 (3572). Colymbus fluviatilis, Tunst. RIciucr Stciñ

a. Hannover. G. r. C. Braunstein.

b. Hannover. G. v. Pralle.

c. pull. Hamover. G. v. Kireye.

d. Hannover. G. v. Raseli.

e. Hamnover.

1354 (3572a). C. poliocephalus (Gould).

i. Australien. (i. v. Konsul kauffmann.

1355 (3574). C. dominicus, L.

a. Brasiliell.

b. juv. Mexiko.

( Mexiko. (i. v. Oberorrt. Dommes.

$1356(3576)$. C. nigricollis (Brehm.) Sdjwar jlualajtcinjul. a. Europad.

1357 (3580). C. (cristatus, L. Senubentandicr.

a. of Hannover. (G. v. I)r. Struckmann.

b. of Hannover. (i. v. Freiherrn Grote.

c. juv. Hannover.

1358 (:3580a). Eudytes septentrionalis (111.).

a. o Nordsee. G. v. Forstmstr. Hesse.

b., c. 2. jur. Spiekeroog.

d. Leer- Ort.

e. Leer-Ort. (i. v. (i. Lohmann.

1:359 (3581). Aechmophorus major (Bodd.).

a. Chili. (i. v. Fabian.

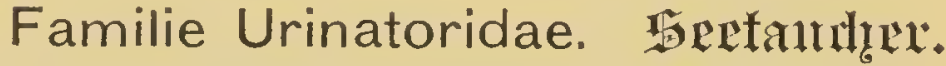

1360 (3584). Urinator areticus (I..) Fivlartnutyer.

a. of jux. Ilaunover. (i. v. ('. Bramenstrin.

1361 (3585). U. toropuatus (13rüm.) (ciztaud)cr.

a. ণै Nordamerikit.

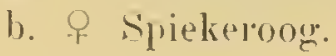

\section{Familie Alcidae.}

1362 (3586). Mergulus alle (1.) Sirabbcutaudicr.

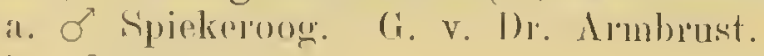

b. o spiekroog.

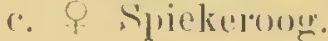


1363 (35sio). Plantus impennis (1..) Fluglojer 9uf.

a. Nordatlantischer ()zean (ausgestorberu).

1364 (3588). Alea tolola, 1. Torbalf.

a. $\sigma$ spickeroog.

b. Of Nordsere.

i. of llelgoland. (i. v. Stolberen.

1. f sipiekroog.

1365 (3589). Uria Iomvia, l. Trottelfunume.

a., b., с. :3 of spiekeroog.

d. $\sigma$ sprekeroog. (i. v. U. Braunstein.

e. Helgoland. G. v. Stolberg.

13366 (3592). Cepphus grylle (L.) Grylltciftc.

a. $\sigma$ Nordsce.

b. \& Nordser.

1367 (3596). Symorhynchus cristatellus (Pall.).

a., b. O' 0 Kamschatka. G. v. Cptn. Graefenhain.

1368 (3599). Fratercula arctica (L.) PBapageitandger.

a. $\sigma$ Nordsee.

b. \& Nordsee.

Ordo Impennes,

\section{Familie Spheniscidae. Binmume.}

1369 (3602). Sphenisens demersus, l. Brifrauinuin.

a. Chili. (G. v. D). Hennecke.

1370 (3602 a). Sph. minor, Gould.

a., b. Australien.

1371 (360)3). Sph. Inagellanicus, Gould.

a., b. suidamerika.

Ordo Brevipennes.

Familie Apterygidae. 筑intit.

1372 (3606). Apteryx mantelli, Bartl. Simi.

a. Neuseeland. (i. v. H. Gröner.

\section{Familie Casuaridae.}

1373 (3608). Hippalectryo (Casuarius!) galeatus (Vicill.) beclutajuar.

a. Ceram. 
1374 (3610). Dromaeus novaehollandiae (Vieill.) (5um.

a. Vandiemensland.

\section{Familie Struthionidae.}

1375 (3711). Rhea rhea (L.) Pandu.

a. Brasilien.

1376 (3612). Struthio camelus (L.) Straur.

a. Afrika.

b. Afrika. 






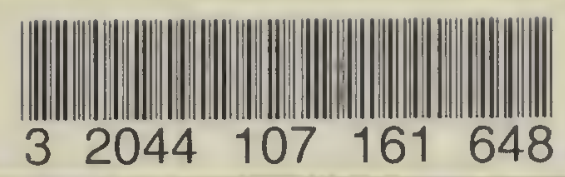




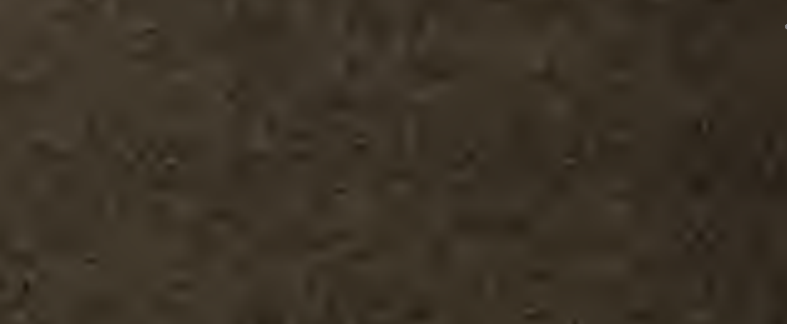

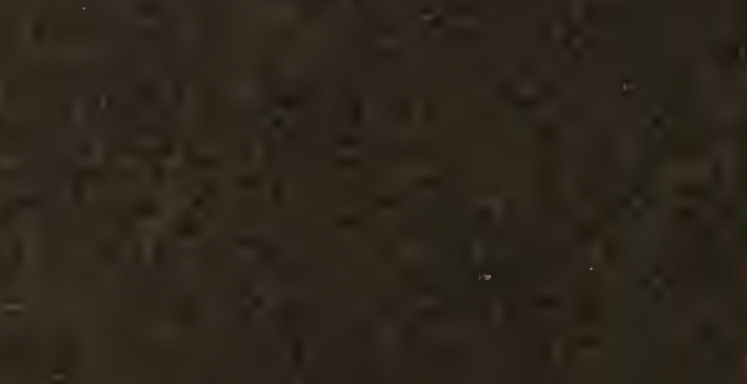

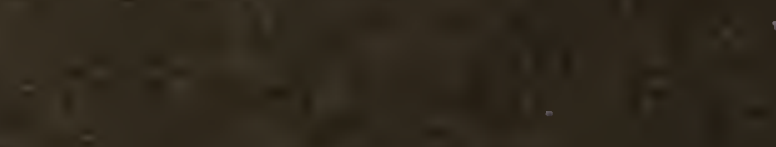

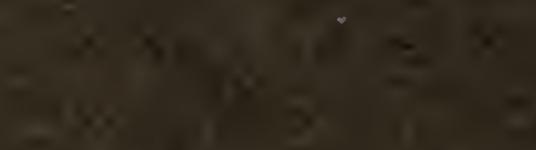

